\title{
Classification of Enriques surfaces with finite automorphism group in characteristic 2
}

\author{
Toshiyuki Katsura, Shigeyuki Kondō and Gebhard Martin
}

\begin{abstract}
We classify supersingular and classical Enriques surfaces with finite automorphism group in characteristic 2 into eight types according to their dual graphs of all $(-2)$ curves (non-singular rational curves). We give examples of these Enriques surfaces together with their canonical coverings. It follows that the classification of all Enriques surfaces with finite automorphism group in any characteristic is finished.
\end{abstract}

\section{Introduction}

We work over an algebraically closed field $k$ of characteristic 2 . The main purpose of this paper is to give a classification of supersingular and classical Enriques surfaces with finite automorphism group in characteristic 2. More precisely, we classify the possible dual graphs of $(-2)$-curves that can occur on these surfaces and give examples realizing each of these graphs. Recall that over the complex numbers, a generic Enriques surface has an infinite group of automorphisms (Barth and Peters [BP83]). On the other hand, Fano [Fan10] and Dolgachev [Dol84] found two examples of Enriques surfaces with finite automorphism group. Then Nikulin [Nik84] proposed a classification of Enriques surfaces with finite automorphism group in terms of periods, and the second author [Kon86] classified and gave constructions of all such Enriques surfaces, geometrically. There are seven types I, II, ..., VII of such Enriques surfaces, distinguished by their dual graphs of $(-2)$ curves. The Enriques surfaces of type I and II form irreducible 1-dimensional families, and each of the remaining types consists of a unique Enriques surface. The first two types contain exactly twelve ( -2$)$-curves (that is, non-singular rational curves), while the remaining five types contain exactly twenty (-2)-curves. The Enriques surfaces of type I (respectively, of type VII) are the examples given by Dolgachev (respectively, by Fano). We call the dual graph of all (-2)-curves on the Enriques surface of type $K$ the dual graph of type $K$, for $K=$ I, II, .., VII. We remark that if an Enriques surface has a dual graph of this type, then its automorphism group is finite.

In positive characteristic, the question of a classification of Enriques surfaces with finite automorphism group has been raised. In particular, the case of characteristic 2 is most interesting. In the paper [BM76], Bombieri and Mumford classified Enriques surfaces in characteristic 2 into three classes, namely singular, classical and supersingular Enriques surfaces. As in the case of

Received 13 September 2017, accepted in final form 14 May 2019.

2010 Mathematics Subject Classification 14J28, 14J50, $14 \mathrm{G} 17$.

Keywords: Enriques surfaces, automorphism groups, $K 3$ surfaces, characteristic 2.

This journal is (C) Foundation Compositio Mathematica 2020. This article is distributed with Open Access under the terms of the Creative Commons Attribution Non-Commercial License, which permits non-commercial reuse, distribution, and reproduction in any medium, provided that the original work is properly cited. For commercial re-use, please contact the Foundation Compositio Mathematica.

Research of the first author is partially supported by Grant-in-Aid for Scientific Research (B) No. 15H03614, that of the second author by (S) No. $15 \mathrm{H} 05738$ and by the Simons Visiting Professorship and that of the third author by the DFG Sachbeihilfe LI 1906/3 - 1 "Automorphismen von Enriques Flächen". 


\section{Classification of EnRiques surfaces}

characteristic 0 or $p>2$, an Enriques surface $X$ in characteristic 2 has a canonical double cover $\pi: \tilde{X} \rightarrow X$, which is a separable $\mathbf{Z} / 2 \mathbf{Z}$-cover or a purely inseparable $\mu_{2}$ or $\alpha_{2}$-cover according to $X$ being singular, classical or supersingular. The surface $\tilde{X}$ might have singularities, and it might even be non-normal, but it is $K 3$-like in the sense that its dualizing sheaf is trivial. Recently, Liedtke [Lie15] showed that the moduli space of Enriques surfaces with a polarization of degree 4 has two 10-dimensional irreducible components. A general point of one component (respectively, the other component) corresponds to a singular (respectively, classical) Enriques surface. The intersection of the two components parametrizes supersingular Enriques surfaces. On the other hand, Ekedahl and Shepherd-Barron [ES04] studied certain special Enriques surfaces called "exceptional Enriques surfaces," whose deformation functors are badly behaved, and Salomonsson [Sal03] gave equations of such Enriques surfaces. We remark that some of them have a finite group of automorphisms.

Very recently, the first and the second author [KK18] determined the existence or nonexistence of Enriques surfaces in characteristic 2 whose dual graphs of all $(-2)$-curves are of type I, II, ..., or VII, as in the following theorem.

Theorem 1.1 (Katsura, Kondo [KK18]). The existence or non-existence of Enriques surfaces in characteristic 2 whose dual graphs of all non-singular rational curves are of type I, II, ... or VII is as in Table 1.

Table 1

\begin{tabular}{|c|c|c|c|c|c|c|c|}
\hline Type & I & II & III & IV & V & VI & VII \\
\hline singular & $\bigcirc$ & $\bigcirc$ & $\times$ & $\times$ & $\times$ & $\bigcirc$ & $\times$ \\
\hline classical & $\times$ & $\times$ & $\times$ & $\times$ & $\times$ & $\times$ & $\bigcirc$ \\
\hline supersingular & $\times$ & $\times$ & $\times$ & $\times$ & $\times$ & $\times$ & $\bigcirc$ \\
\hline
\end{tabular}

In Table 1 and in the following Table 2, $\bigcirc$ means the existence and $\times$ means the non-existence of an Enriques surface with dual graph of type I, ..., VII.

On the other hand, the third author [Mar20] gave a classification of Enriques surfaces with finite automorphism group in characteristic $p>2$ and in the case of singular Enriques surfaces by using the method given by the second author over the complex numbers.

Theorem 1.2 (Martin [Mar20]). Table 2 gives the classification of Enriques surfaces with finite automorphism group whose canonical coverings are smooth. The moduli spaces for type I and II are 1-dimensional and irreducible. For each of the other types, there is a unique such surface.

Table 2

\begin{tabular}{|c|c|c|c|c|c|c|c|}
\hline Type & $\mathrm{I}$ & II & III & IV & V & VI & VII \\
\hline$p=0$ or $p>5$ & $\bigcirc$ & $\bigcirc$ & $\bigcirc$ & $\bigcirc$ & $\bigcirc$ & $\bigcirc$ & $\bigcirc$ \\
\hline$p=5$ & $\bigcirc$ & $\bigcirc$ & $\bigcirc$ & $\bigcirc$ & $\bigcirc$ & $\times$ & $\times$ \\
\hline$p=3$ & $\bigcirc$ & $\bigcirc$ & $\bigcirc$ & $\bigcirc$ & $\times$ & $\times$ & $\bigcirc$ \\
\hline$p=2$, singular & $\bigcirc$ & $\bigcirc$ & $\times$ & $\times$ & $\times$ & $\bigcirc$ & $\times$ \\
\hline
\end{tabular}

Thus, the classification problem remains only for the classical and supersingular cases. Now, we state the main results of this paper. In Theorems 1.3(b) and 1.4(b), we give examples of 


\section{T. Katsura, S. Kondō And G. Martin}

Enriques surfaces with finite automorphism groups. Some of them are constructed as families of such Enriques surfaces. In Tables 3 and 4, "dim" denotes the dimension of such a family of examples. The families of type $\tilde{E}_{7}+\tilde{A}_{1}^{(1)}$ supersingular, of type $\tilde{E}_{6}+\tilde{A}_{2}$ classical, of type VII classical and of type VIII are non-isotrivial. The family of type $\tilde{E}_{7}+\tilde{A}_{1}^{(1)}$ classical surfaces and the family of type $\tilde{D}_{4}+\tilde{D}_{4}$ contain an at least 1-dimensional non-isotrivial family. The authors do not know of the existence of other examples; that is, the problem of determining the moduli space of such Enriques surfaces is still open. We denote by $\operatorname{Aut}(X), \operatorname{Aut}_{\text {ct }}(X)$ and $\operatorname{Aut}_{n t}(X)$ the automorphism group of $X$, the cohomologically trivial automorphism group and the numerically trivial automorphism group (see Definition 2.3), respectively. Let $\mathfrak{S}_{n}$ be the symmetric group of degree $n$ and $Q_{8}$ the quaternion group of order 8.

Theorem 1.3. Let $X$ be a supersingular Enriques surface in characteristic 2.

(a) The surface $X$ has a finite group of automorphisms if and only if the dual graph of all $(-2)$-curves on $X$ is one of the graphs in Table $3(\mathrm{a})$.

(b) There exist families of these surfaces with the automorphism groups and dimensions given in Table 3(b).

Table 3

(a) Classification

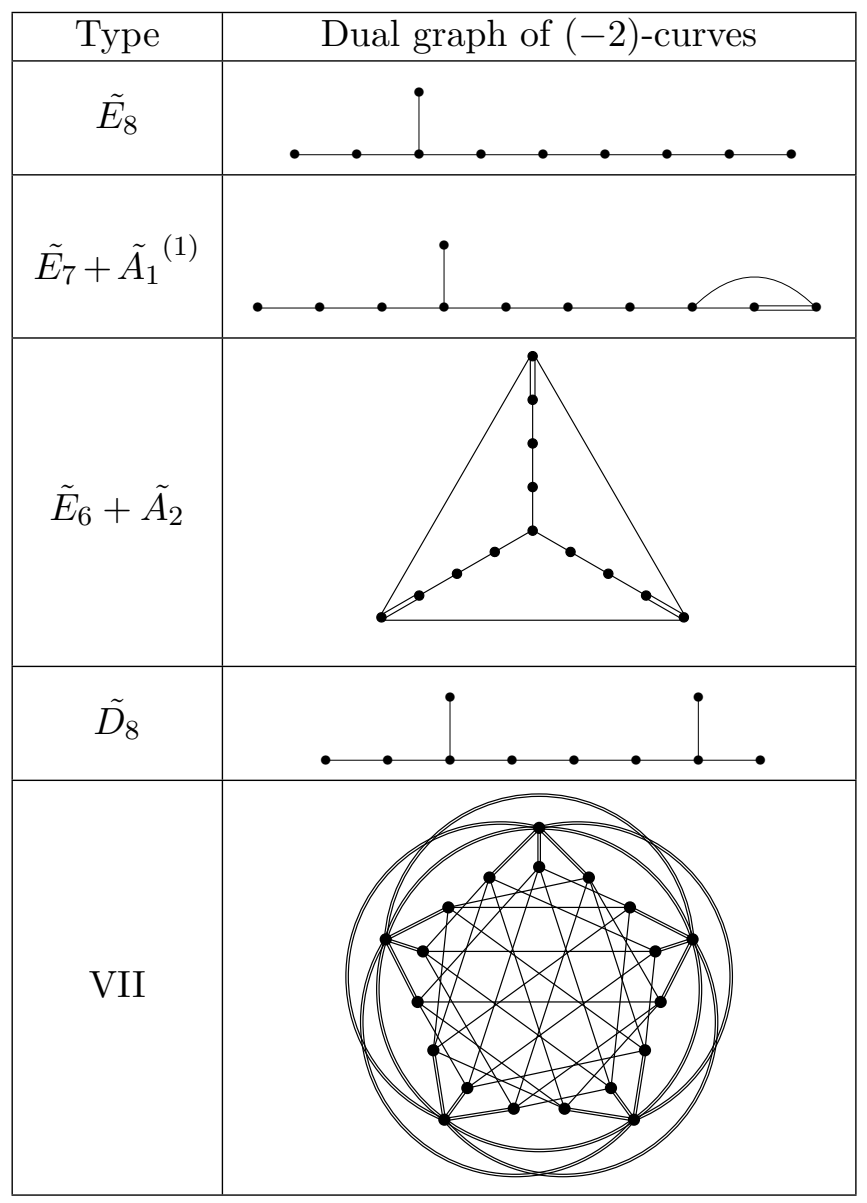

(b) Examples

\begin{tabular}{|c|c|c|}
\hline $\operatorname{Aut}(X)$ & $\operatorname{Aut}_{c t}(X)$ & $\operatorname{dim}$ \\
\hline $\mathbf{Z} / 11 \mathbf{Z}$ & $\mathbf{Z} / 11 \mathbf{Z}$ & 0 \\
\hline $\begin{array}{c}\mathbf{Z} / 2 \mathbf{Z} \text { or } \\
\mathbf{Z} / 14 \mathbf{Z}\end{array}$ & $\begin{array}{l}\{1\} \text { or } \\
\mathbf{Z} / 7 \mathbf{Z}\end{array}$ & $\begin{array}{c}1 \text { or } \\
0\end{array}$ \\
\hline $\mathbf{Z} / 5 \mathbf{Z} \times \mathfrak{S}_{3}$ & $\mathbf{Z} / 5 \mathbf{Z}$ & 0 \\
\hline$Q_{8}$ & $Q_{8}$ & 1 \\
\hline $\mathfrak{S}_{5}$ & $\{1\}$ & 0 \\
\hline
\end{tabular}




\section{Classification of EnRiques surfaces}

Theorem 1.4. Let $X$ be a classical Enriques surface in characteristic 2 .

(a) The surface $X$ has a finite group of automorphisms if and only if the dual graph of all $(-2)$-curves on $X$ is one of the graphs in Table 4(a).

(b) There exist families of these surfaces with the automorphism groups and dimensions given in Table 4(b).

Table 4

(a) Classification

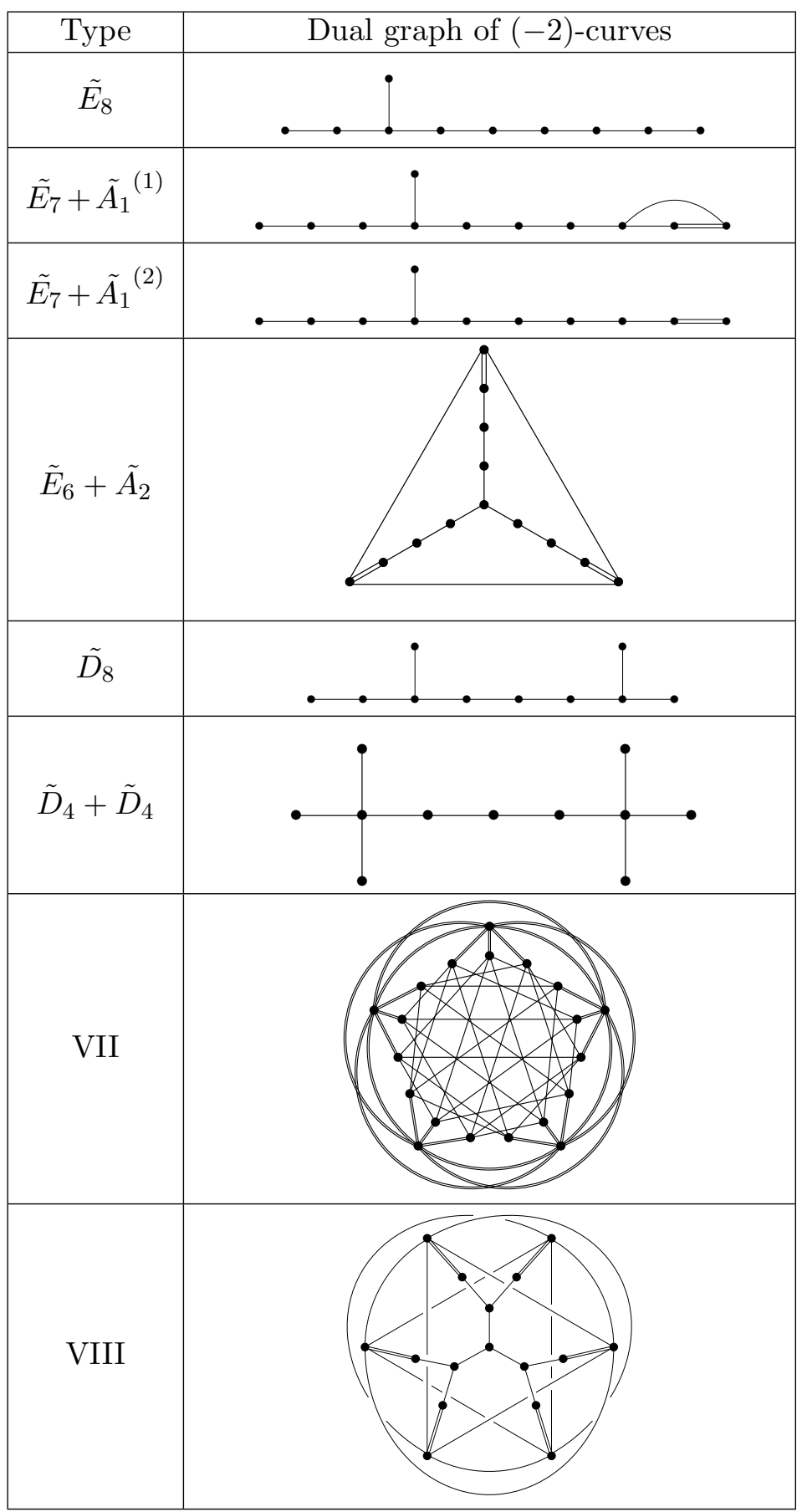

(b) Examples

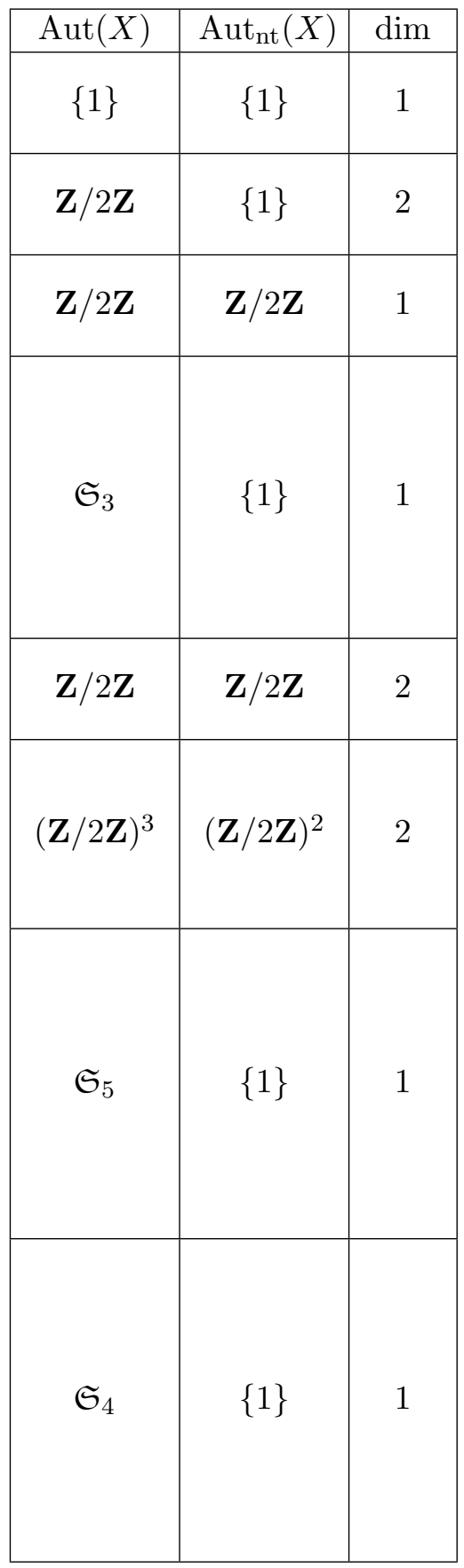




\section{T. Katsura, S. Kondō And G. Martin}

We remark that the examples of supersingular Enriques surfaces of type $\tilde{E}_{7}+\tilde{A}_{1}^{(1)}$ form a 1 dimensional family, but some of their automorphism groups jump up.

Over the complex numbers, Enriques surfaces with numerically and cohomologically trivial automorphism groups are completely classified into three types, and the groups are cyclic of order 2 or 4 (Mukai and Namikawa [MN84], Mukai [Muk10] and see also Kondō [Kon86, Theorem 1.7]). This is not true in characteristic 2, and Theorems 1.3(b) and 1.4(b) give new examples of such automorphisms (that is, the cases $\tilde{E}_{8}$ supersingular, $\tilde{E}_{7}+\tilde{A}_{1}^{(1)}$ supersingular, $\tilde{E}_{6}+\tilde{A}_{2}$ supersingular, $\tilde{D}_{8}$ supersingular and $\tilde{D}_{4}+\tilde{D}_{4}$ classical); compare with Dolgachev [Dol13, Theorem 4]. Very recently, Dolgachev and the third author [DM19] gave a classification of the possible numerically and cohomologically trivial automorphism groups of Enriques surfaces in positive characteristic up to the examples in Theorems 1.3 and 1.4. In particular, they show that nothing new happens in characteristic different from 2.

Corollary 1.5. For $G \in\left\{Q_{8}, \mathbf{Z} / 11 \mathbf{Z}, \mathbf{Z} / 7 \mathbf{Z}, \mathbf{Z} / 5 \mathbf{Z}\right\}$, there exists a supersingular Enriques surface $X$ with $\operatorname{Aut}_{\mathrm{ct}}(X)=G$. Moreover, there is a classical Enriques surface $X$ in characteristic 2 with $\operatorname{Aut}_{n t}(X)=\mathbf{Z} / 2 \mathbf{Z} \times \mathbf{Z} / 2 \mathbf{Z}$.

Note that only the dual graph of type VII in Theorems 1.3 and 1.4 appears over the complex numbers. Moreover, the Enriques surface with dual graph of type VII is unique over the complex numbers, whereas our example in characteristic 2 is a 1-dimensional and non-isotrivial family of classical and supersingular Enriques surfaces with such a dual graph (see Theorem 8.1). The canonical cover of any Enriques surface of type VII has twelve rational double points of type $A_{1}$, and its minimal resolution is the unique supersingular $K 3$ surface with Artin invariant 1 . The canonical covers of the other Enriques surfaces in Theorems 1.3 and 1.4 are non-normal rational surfaces.

The dual graphs of type $\tilde{E}_{8}, \tilde{E}_{7}+\tilde{A}_{1}^{(1)}, \tilde{E}_{7}+\tilde{A}_{1}^{(2)}, \tilde{D}_{8}$ and $\tilde{D}_{4}+\tilde{D}_{4}$ appeared in Cossec and Dolgachev [CD89] and in unpublished work by I. V. Dolgachev and C. Liedtke, and the first four are called "extra special." Also, Enriques surfaces of type $\tilde{E}_{8}, \tilde{E}_{7}+\tilde{A}_{1}^{(1)}, \tilde{E}_{7}+\tilde{A}_{1}^{(2)}$ or $\tilde{E}_{6}+\tilde{A}_{2}$ are called "exceptional" and were studied in detail by Ekedahl and Shepherd-Barron [ES04] and Salomonsson [Sal03] from a different point of view. In particular, Salomonsson first constructed these exceptional surfaces. However, the existence of Enriques surfaces of type $\tilde{D}_{8}$ or $\tilde{D}_{4}+\tilde{D}_{4}$ was not known before our classification.

In the case of a classical or supersingular Enriques surface $X$, there exists a non-zero regular global 1-form $\eta$ on $X$. The divisorial part of the scheme of zeros of $\eta$ is called the bi-conductrix and the half of the bi-conductrix the conductrix. By definition, the canonical cover $\pi: \tilde{X} \rightarrow X$ has a singularity at $P \in \tilde{X}$ if and only if $\eta$ vanishes at $\pi(P)$. Ekedahl and Shepherd-Barron [ES04] classified possible conductrices of elliptic and quasi-elliptic fibrations on classical and supersingular Enriques surfaces. The conductrix is an additional invariant of Enriques surfaces with non-normal cover; it will play a central role in our classification.

The outline of the proof of Theorems 1.3 and 1.4 is as follows. First, recall that any Enriques surface $X$ admits a genus 1 fibration $\pi: X \rightarrow \mathbf{P}^{1}$, and any genus 1 fibration on an Enriques surface has a double fiber. Let $J(\pi): J(X) \rightarrow \mathbf{P}^{1}$ be the Jacobian fibration associated with $\pi$. Then the Mordell-Weil group of $J(\pi)$ acts on $X$ effectively as automorphisms. Now, assume that the automorphism group $\operatorname{Aut}(X)$ is finite. Then, for any genus 1 fibration $\pi$ on $X$, the MordellWeil rank of its Jacobian fibration is 0 (see Proposition 2.10). We will prove that the possible dual graphs of (-2)-curves on $X$ are nothing but those given in Theorems 1.3(a) and 1.4(a), by using the condition on the Mordell-Weil rank mentioned above and Ekedahl and Shepherd-Barron's 


\section{Classification of Enriques surfaces}

classification of conductrices (Theorem 4.1). Then it follows from a result by Vinberg [Vin75] that $\operatorname{Aut}(X)$ is in fact finite for every Enriques surface $X$ with one of these dual graphs of $(-2)$-curves (Proposition 2.14).

On the other hand, for each dual graph $\Gamma$ in Theorems 1.3(a) and 1.4(a), we will construct Enriques surfaces with $\Gamma$ as the dual graph of (-2)-curves (Sections 7-13). To do this, we look at a subdiagram $\Gamma_{0}$ of $\Gamma$ which is the dual graph of reducible fibers of a special genus 1 fibration. Here, a genus 1 fibration is called special if the fibration has a (-2)-curve as a 2 -section. We first consider a rational genus 1 fibration $g: R \rightarrow \mathbf{P}^{1}$ whose dual graph of reducible fibers is $\Gamma_{0}$, and we take the Frobenius base change $f: \tilde{R} \rightarrow \mathbf{P}^{1}$ of $g$. Then, we construct a rational vector field $D$ on $\tilde{R}$ (in Section 5 , we give a method to find suitable vector fields). The vector field $D$ might have isolated singularities, and hence we take a resolution of singularities; that is, after blow-ups of $\tilde{R}$, we get a non-singular surface $Y$ such that the induced vector field, denoted by the same symbol $D$, has no isolated singularities. Then, the quotient surface $Y^{D}$ of $Y$ by $D$ is non-singular, and the minimal model $X$ of $Y^{D}$ is the desired Enriques surface.

Finally, we make a remark on how to calculate the automorphism group $\operatorname{Aut}(X)$, which is isomorphic to a subgroup of the symmetry group of the dual graph $\Gamma$ up to numerically trivial automorphisms. In the cases $\tilde{E}_{6}+\tilde{A}_{2}$ (supersingular), $\tilde{E}_{8}$ (supersingular and classical), $\tilde{E}_{7}+\tilde{A}_{1}^{(1)}$ (supersingular), $\tilde{D}_{8}$ (supersingular and classical) and $\tilde{D}_{4}+\tilde{D}_{4}$, we cannot determine the numerically trivial automorphisms from the dual graphs of $(-2)$-curves geometrically. In these cases, we first find an equation of an affine surface birationally equivalent to $X$, and then we reduce the problem to the calculation of the automorphism group of this surface (see Section 6).

In Sections 3 through 12, unless mentioned otherwise, all our Enriques surfaces are classical or supersingular.

\section{Preliminaries}

\subsection{Vector fields}

Let $k$ be an algebraically closed field of characteristic $p>0$, and let $S$ be a non-singular complete algebraic surface defined over $k$. We denote by $K_{S}$ a canonical divisor of $S$. A rational vector field $D$ on $S$ is said to be $p$-closed if there exists a rational function $f$ on $S$ such that $D^{p}=f D$. A vector field $D$ is of additive type (respectively, of multiplicative type) if $D^{p}=0$ (respectively, $\left.D^{p}=D\right)$. Let $\left\{U_{i}=\operatorname{Spec} A_{i}\right\}$ be an affine open covering of $S$. We set $A_{i}^{D}=\left\{\alpha \in A_{i} \mid D(\alpha)=0\right\}$. The affine varieties $\left\{U_{i}^{D}=\operatorname{Spec} A_{i}^{D}\right\}$ glue together to define a normal quotient surface $S^{D}$.

Now, we assume that $D$ is $p$-closed. Then, the natural morphism

$$
\pi: S \longrightarrow S^{D}
$$

is a purely inseparable morphism of degree $p$. If the affine open covering $\left\{U_{i}\right\}$ of $S$ is fine enough, then taking local coordinates $x_{i}, y_{i}$ on $U_{i}$, we see that there exist $g_{i}, h_{i} \in A_{i}$ and a rational function $f_{i}$ such that the divisors defined by $g_{i}=0$ and by $h_{i}=0$ have no common components, and such that

$$
D=f_{i}\left(g_{i} \frac{\partial}{\partial x_{i}}+h_{i} \frac{\partial}{\partial y_{i}}\right) \text { on } U_{i} .
$$

By Rudakov and Shafarevich [RS76, Section 1], the divisors $\left(f_{i}\right)$ on $U_{i}$ give a global divisor $(D)$ on $S$, and the zero-cycles defined by the ideal $\left(g_{i}, h_{i}\right)$ on $U_{i}$ give a global zero cycle $\langle D\rangle$ on $S$. A point contained in the support of $\langle D\rangle$ is called an isolated singular point of $D$. If $D$ has no isolated singular point, $D$ is said to be divisorial. Rudakov and Shafarevich $[\mathrm{RS} 76$, Theorem 1 , 


\section{T. Katsura, S. Kondō And G. Martin}

Corollary] showed that $S^{D}$ is non-singular if $\langle D\rangle=0$; that is, $D$ is divisorial. When $S^{D}$ is non-singular, they also showed a canonical divisor formula

$$
K_{S} \sim \pi^{*} K_{S^{D}}+(p-1)(D),
$$

where $\sim$ means linear equivalence. As for the Euler number $c_{2}(S)$ of $S$, we have a formula

$$
c_{2}(S)=\operatorname{deg}\langle D\rangle-K_{S} \cdot(D)-(D)^{2}
$$

(cf. Katsura and Takeda [KT89, Proposition 2.1]).

Now we consider an irreducible curve $C$ on $S$, and we set $C^{\prime}=\pi(C)$. Take an affine open set $U_{i}$ as above such that $C \cap U_{i}$ is non-empty. The curve $C$ is said to be integral with respect to the vector field $D$ if $g_{i} \partial / \partial x_{i}+h_{i} \partial / \partial y_{i}$ is tangent to $C$ at a general point of $C \cap U_{i}$. Then, Rudakov-Shafarevich [RS76, Proposition 1] showed the following proposition.

Proposition 2.1. (i) If $C$ is integral, then $C=\pi^{*}\left(C^{\prime}\right)$ and $C^{2}=p C^{\prime 2}$.

(ii) If $C$ is not integral, then $p C=\pi^{*}\left(C^{\prime}\right)$ and $p C^{2}=C^{\prime 2}$.

\subsection{Enriques surfaces in characteristic 2}

In characteristic 2, a minimal algebraic surface with numerically trivial canonical divisor is called an Enriques surface if the second Betti number is equal to 10. Such surfaces $X$ are divided into three classes (for details, see Bombieri and Mumford [BM76, Section 3]):

(1) $K_{X}$ is not linearly equivalent to zero and $2 K_{X} \sim 0$. Such an Enriques surface is called a classical Enriques surface.

(2) $K_{X} \sim 0, \mathrm{H}^{1}\left(X, \mathcal{O}_{X}\right) \cong k$ and the Frobenius map acts on $\mathrm{H}^{1}\left(X, \mathcal{O}_{X}\right)$ bijectively. Such an Enriques surface is called a singular Enriques surface.

(3) $K_{X} \sim 0, \mathrm{H}^{1}\left(X, \mathcal{O}_{X}\right) \cong k$ and the Frobenius map is the zero map on $\mathrm{H}^{1}\left(X, \mathcal{O}_{X}\right)$. Such an Enriques surface is called a supersingular Enriques surface.

It is known that $\operatorname{Pic}_{X}^{\tau}$ is isomorphic to $\mathbf{Z} / 2 \mathbf{Z}$ if $X$ is classical, to $\mu_{2}$ if $X$ is singular and to $\alpha_{2}$ if $X$ is supersingular (Bombieri-Mumford [BM76, Theorem 2]). As in the case of characteristic 0 or $p>2$, an Enriques surface $X$ in characteristic 2 has a canonical double cover $\pi: \tilde{X} \rightarrow X$, which is a separable $\mathbf{Z} / 2 \mathbf{Z}$-cover or a purely inseparable $\mu_{2^{-}}$or $\alpha_{2}$-cover according to $X$ being singular, classical or supersingular. The surface $\tilde{X}$ might have singularities, and it might even be non-normal (see Proposition 3.1), but it is $K 3$-like in the sense that its dualizing sheaf is trivial and $\mathrm{H}^{1}\left(\tilde{X}, \mathcal{O}_{\tilde{X}}\right)=0$. Note that Ekedahl and Shepherd-Barron [ES04] use the term "unipotent" Enriques surface for supersingular and classical ones.

\section{$2.3(-2)$-curves}

Let $X$ be an Enriques surface, and let $\operatorname{Num}(X)$ be the quotient of the Néron-Severi group $\operatorname{NS}(X)$ of $X$ by its torsion subgroup. Then $\operatorname{Num}(X)$ together with the intersection product is an even unimodular lattice of signature $(1,9)$ (Illusie [Ill79, Corollary 7.3.7]) and hence is isomorphic to $U \oplus E_{8}$, where $U$ is the even unimodular lattice of signature $(1,1)$ and $E_{8}$ the even negative-definite unimodular lattice of rank 8 . We denote by $\mathrm{O}(\mathrm{Num}(X))$ the orthogonal group of $\operatorname{Num}(X)$. The set

$$
\{x \in \operatorname{Num}(X) \otimes \mathbf{R}:\langle x, x\rangle>0\}
$$




\section{Classification of EnRiques surfaces}

has two connected components. Denote by $P(X)$ the connected component containing an ample class of $X$. For $\delta \in \operatorname{Num}(X)$ with $\delta^{2}=-2$, we define an isometry $s_{\delta}$ of $\operatorname{Num}(X)$ by

$$
s_{\delta}(x)=x+\langle x, \delta\rangle \delta, \quad x \in \operatorname{Num}(X),
$$

which is nothing but the reflection with respect to the hyperplane perpendicular to $\delta$. The isometry $s_{\delta}$ is called the reflection associated with $\delta$. We call a non-singular rational curve on an Enriques surface or a $K 3$ surface a $(-2)$-curve. For a $(-2)$-curve $E$ on an Enriques surface $X$, we identify $E$ with its class in $\operatorname{Num}(X)$. Let $W(X)$ be the subgroup of $\mathrm{O}(\operatorname{Num}(X))$ generated by reflections associated with all $(-2)$-curves on $X$. Then $P(X)$ is divided into chambers each of which is a fundamental domain with respect to the action of $W(X)$ on $P(X)$. There exists a unique chamber containing an ample class which is nothing but the closure of the ample cone $D(X)$ of $X$. It is known that the natural map

$$
\rho_{n}: \operatorname{Aut}(X) \rightarrow \mathrm{O}(\operatorname{Num}(X))
$$

has a finite kernel. Since the image $\operatorname{Im}\left(\rho_{n}\right)$ preserves the ample cone, we see that $\operatorname{Im}\left(\rho_{n}\right) \cap W(X)=$ $\{1\}$. Therefore, $\operatorname{Aut}(X)$ is finite if the index $[\mathrm{O}(\operatorname{Num}(X)): W(X)]$ is finite. Thus, we have the following proposition (see Dolgachev [Dol84, Proposition 3.2]).

Proposition 2.2. If $W(X)$ is of finite index in $\mathrm{O}(\operatorname{Num}(X))$, then $\operatorname{Aut}(X)$ is finite.

Over the field of complex numbers, the converse of Proposition 2.2 holds, as can be seen by using the Torelli-type theorem for Enriques surfaces (Dolgachev [Dol84, Theorem 3.3]).

Definition 2.3. Denote by $\operatorname{Aut}_{n t}(X)$ the kernel of the map $\rho_{n}$ given by (2.4). Similarly, denote by $\operatorname{Aut}_{\mathrm{ct}}(X)$ the kernel of the map

$$
\rho_{c}: \operatorname{Aut}(X) \rightarrow \mathrm{O}(\mathrm{NS}(X)) .
$$

A non-trivial automorphism is called cohomologically or numerically trivial if it is contained in $\operatorname{Aut}_{\mathrm{ct}}(X)$ or $\operatorname{Aut}_{\mathrm{nt}}(X)$, respectively. If $S$ is not classical, then $\mathrm{NS}(X)=\operatorname{Num}(X)$ and hence $\operatorname{Aut}_{\mathrm{ct}}(X)=\operatorname{Aut}_{\mathrm{nt}}(X)$.

\subsection{Genus 1 fibrations}

We recall some facts on elliptic and quasi-elliptic fibrations on Enriques surfaces. For simplicity, we call an elliptic or a quasi-elliptic fibration a genus 1 fibration.

Proposition 2.4 (Bombieri and Mumford [BM76, Theorem 3]). Every Enriques surface $S$ has a genus 1 fibration. Conversely, for any primitive nef isotropic divisor $D$ on $S$, the linear system $|D|$ or $|2 D|$ defines a genus 1 fibration on $S$.

Proposition 2.5 (Cossec and Dolgachev [CD89, Theorems 5.7.5 and 5.7.6]). Let $f: X \rightarrow \mathbf{P}^{1}$ be a genus 1 fibration on an Enriques surface $X$ in characteristic 2. Then, the following hold.

(i) If $X$ is classical, then $f$ has two tame double fibers, each of which is either an ordinary elliptic curve or a singular fiber of additive type.

(ii) If $X$ is singular, then $f$ has one wild double fiber, which is a smooth ordinary elliptic curve or a singular fiber of multiplicative type.

(iii) If $X$ is supersingular, then $f$ has one wild double fiber, which is a supersingular elliptic curve or a singular fiber of additive type. 


\section{T. Katsura, S. Kondō And G. Martin}

Lemma 2.6. Let $f: X \rightarrow \mathbf{P}^{1}$ be an isotrivial genus 1 fibration on an Enriques surface in characteristic 2. Let $F$ be a double fiber of $f$ such that the underlying reduced fiber $F_{\text {red }}$ is an elliptic curve. Then $F_{\text {red }}$ has $j$-invariant 0 if and only if the generic fiber of $f$ also has $j$-invariant 0 .

Proof. We can assume that the general fiber of $f$ is an elliptic curve. Since $f$ is isotrivial, it becomes trivial after passing to a finite cover of $\mathbf{P}^{1}$. Hence $F$ is isogeneous to the generic fiber of $f$. Since having $j$-invariant 0 is equivalent to being supersingular in characteristic 2 and being supersingular is an isogeny-invariant, we get the result.

We use the symbols $\mathrm{I}_{n}(n \geqslant 1), \mathrm{I}_{n}^{*}(n \geqslant 0)$, II, III, IV, II* $\mathrm{III}^{*}, \mathrm{IV}^{*}$ of singular fibers of an elliptic or a quasi-elliptic fibration in the sense of Kodaira. The dual graph of $(-2)$-curves in a singular fiber of type $\mathrm{I}_{n}(n \geqslant 2), \mathrm{I}_{n}^{*}(n \geqslant 0)$, III, IV, II* III $^{*}$ or IV $^{*}$ is an extended Dynkin diagram $\tilde{A}_{n-1}, \tilde{D}_{n+4}, \tilde{A}_{1}, \tilde{A}_{2}, \tilde{E}_{8}, \tilde{E}_{7}$ or $\tilde{E}_{6}$, respectively. For a double singular fiber of type $F$, we write $2 F$. Let $f: S \rightarrow \mathbf{P}^{1}$ be a genus 1 fibration on a surface $S$. If, for example, $f$ has a double singular fiber of type III and a singular fiber of type IV $^{*}$, then we say that $f$ has singular fibers $\left(2 \mathrm{III}, \mathrm{IV}^{*}\right)$. If $f$ has a section and its Mordell-Weil group is torsion, then $f$ is called extremal. We use the following classifications of extremal rational elliptic and rational quasi-elliptic fibrations.

Proposition 2.7 (Lang [Lan91, Lan94]). The following are the singular fibers of extremal elliptic fibrations on rational surfaces in characteristic 2 :

$$
\begin{aligned}
& \left(\mathrm{II}^{*}\right),\left(\mathrm{II}^{*}, \mathrm{I}_{1}\right),\left(\mathrm{III}^{*}, \mathrm{I}_{2}\right),\left(\mathrm{IV}^{*}, \mathrm{IV}\right),\left(\mathrm{IV}^{*}, \mathrm{I}_{3}, \mathrm{I}_{1}\right),\left(\mathrm{I}_{4}^{*}\right),\left(\mathrm{I}_{1}^{*}, \mathrm{I}_{4}\right), \\
& \left(\mathrm{I}_{9}, \mathrm{I}_{1}, \mathrm{I}_{1}, \mathrm{I}_{1}\right),\left(\mathrm{I}_{8}, \mathrm{III}\right),\left(\mathrm{I}_{6}, \mathrm{IV}, \mathrm{I}_{2}\right),\left(\mathrm{I}_{5}, \mathrm{I}_{5}, \mathrm{I}_{1}, \mathrm{I}_{1}\right),\left(\mathrm{I}_{3}, \mathrm{I}_{3}, \mathrm{I}_{3}, \mathrm{I}_{3}\right) .
\end{aligned}
$$

Proposition 2.8 (Ito [Ito94]). The following are the singular fibers of quasi-elliptic fibrations on rational surfaces in characteristic 2 :

$$
\begin{gathered}
\left(\mathrm{II}^{*}\right),\left(\mathrm{III} \mathrm{I}^{*}, \mathrm{III}\right),\left(\mathrm{I}_{4}^{*}\right),\left(\mathrm{I}_{2}^{*}, \mathrm{III}, \mathrm{III}\right),\left(\mathrm{I}_{0}^{*}, \mathrm{I}_{0}^{*}\right), \\
\left(\mathrm{I}_{0}^{*}, \mathrm{III}, \mathrm{III}, \mathrm{III}, \mathrm{III}\right),(\mathrm{III}, \mathrm{III}, \mathrm{III}, \mathrm{III}, \mathrm{III}, \mathrm{III}, \mathrm{III}, \mathrm{III}) .
\end{gathered}
$$

Remark 2.9. Any quasi-elliptic fibration on a rational surface is extremal. This follows from the fact that any section of a quasi-elliptic fibration with a section is of finite order (cf. Ito [Ito92, Theorems 2.2 and 2.4 and Corollary 2.12]).

Consider a genus 1 fibration on an Enriques surface $\pi: X \rightarrow \mathbf{P}^{1}$. Then the Mordell-Weil group of the Jacobian of $\pi$ acts on $X$ effectively as automorphisms. This implies the following proposition.

Proposition 2.10 (Dolgachev [Dol84, Section 4]). Assume that the automorphism group of an Enriques surface $X$ is finite. Then any genus 1 fibration on $X$ is extremal.

Let $X$ be an Enriques surface. A genus 1 fibration $f: X \rightarrow \mathbf{P}^{1}$ is called special if there exists a (-2)-curve $R$ with $R \cdot f^{-1}(P)=2\left(P \in \mathbf{P}^{1}\right)$, that is, $f$ has a $(-2)$-curve as a 2 -section. In this case, $R$ is called a special 2-section. Note that for any quasi-elliptic fibration, the locus of singular points of irreducible fibers gives a special 2-section of the fibration. We call this special 2 -section the curve of cusps of the fibration. The following result is due to Cossec [Cos85]; he assumed that the characteristic $p$ is different from 2 , but the assertion also holds for $p=2$.

Proposition 2.11 (Lang [Lan88, Theorem A3]). Assume that an Enriques surface $X$ contains a (-2)-curve. Then there exists a special genus 1 fibration on $X$. 


\section{Classification of EnRiques surfaces}

Remark 2.12. Any quasi-elliptic fibration is special because the curve of cusps (that is, the curve consisting of the cusps of the fibers) is a special 2-section.

For future reference, let us state the following well-known facts about automorphisms of curves of arithmetic genus 1 (compare with, for example, [DM19]).

Lemma 2.13. Let $C$ be a curve of arithmetic genus 1 over an algebraically closed field of characteristic 2 , and let id $\neq g \in \operatorname{Aut}(C)$ be an automorphism of finite order.

(i) If $g$ has even (respectively, odd) order and $C$ is smooth, then $g$ fixes at most two (respectively, three) points on $C$.

(ii) If $g$ has even (respectively, odd) order and $C$ is cuspidal, then $g$ fixes exactly one (respectively, two) points on $C$.

\subsection{Vinberg's criterion}

Let $X$ be an Enriques surface. We recall Vinberg's criterion, which guarantees that a group generated by a finite number of reflections is of finite index in $\mathrm{O}(\mathrm{Num}(X))$.

Let $\Delta$ be a finite set of $(-2)$-vectors in $\operatorname{Num}(X)$. Let $\Gamma$ be the graph of $\Delta$; that is, $\Delta$ is the set of vertices of $\Gamma$, and two vertices $\delta$ and $\delta^{\prime}$ are joined by $m$-tuple lines if $\left\langle\delta, \delta^{\prime}\right\rangle=m$. We assume that the cone

$$
K(\Gamma)=\left\{x \in \operatorname{Num}(X) \otimes \mathbf{R}:\left\langle x, \delta_{i}\right\rangle \geqslant 0, \delta_{i} \in \Delta\right\}
$$

is a strictly convex cone. Such a $\Gamma$ is called non-degenerate. A connected parabolic subdiagram $\Gamma^{\prime}$ in $\Gamma$ is a Dynkin diagram of type $\tilde{A}_{m}, \tilde{D}_{n}$ or $\tilde{E}_{k}$ (see Vinberg [Vin75, Table 2, p. 345]). If the number of vertices of $\Gamma^{\prime}$ is $r+1$, then $r$ is called the rank of $\Gamma^{\prime}$. A disjoint union of connected parabolic subdiagrams is called a parabolic subdiagram of $\Gamma$. We denote by $\tilde{K}_{1} \oplus \cdots \oplus \tilde{K}_{s}$ a parabolic subdiagram which is a disjoint union of connected parabolic subdiagrams of type $\tilde{K}_{1}, \ldots, \tilde{K}_{s}$, where $K_{i}$ is $A_{m}, D_{n}$ or $E_{k}$. The rank of a parabolic subdiagram is the sum of the ranks of its connected components. Note that the dual graph of reducible singular fibers of a genus 1 fibration on $X$ gives a parabolic subdiagram. We denote by $W(\Gamma)$ the subgroup of $\mathrm{O}(\operatorname{Num}(X))$ generated by the reflections associated with $\delta \in \Gamma$.

Proposition 2.14 (Vinberg [Vin75, Theorem 2.3]). Let $\Delta$ be a set of (-2)-vectors in $\operatorname{Num}(X)$, and let $\Gamma$ be the graph of $\Delta$. Assume that $\Delta$ is a finite set, $\Gamma$ is non-degenerate and $\Gamma$ contains no $m$-tuple lines with $m \geqslant 3$. Then $W(\Gamma)$ is of finite index in $\mathrm{O}(\operatorname{Num}(X))$ if and only if every connected parabolic subdiagram of $\Gamma$ is a connected component of some parabolic subdiagram in $\Gamma$ of rank 8 (namely, the maximal one).

Remark 2.15. Note that $\Gamma$ as in Proposition 2.14 is automatically non-degenerate if it contains the components of the reducible fibers of a special extremal genus 1 fibration and a special 2section of this fibration. Indeed, these curves will generate $\operatorname{Num}(X) \otimes \mathbf{Q}$, and hence $K(\Gamma)$ is strictly convex.

Proposition 2.16 (Namikawa [Nam85, Proposition 6.9]). Let $\Delta$ be a finite set of (-2)-curves on an Enriques surface $X$, and let $\Gamma$ be the graph of $\Delta$. Assume that $W(\Gamma)$ is of finite index in $\mathrm{O}(\operatorname{Num}(X))$. Then $\Delta$ is the set of all $(-2)$-curves on $X$. 


\section{T. Katsura, S. Kondō And G. Martin}

\section{Conductrix}

Let $X$ be a classical or supersingular Enriques surface. Then it is known that there exists a global regular 1-form $\eta$ on $X$. The canonical cover $\pi: \tilde{X} \rightarrow X$ has a singularity at $P \in \tilde{X}$ if and only if $\eta$ vanishes at $\pi(P)$. Since $c_{2}(X)=12$, the 1 -form $\eta$ always vanishes somewhere, and hence $\tilde{X}$ is singular. The divisorial part $B$ of the zero scheme of $\eta$ is called the bi-conductrix of $X$. The divisor $B$ is of the form $2 A$, where $A$ is a divisor called the conductrix of $X$.

The purpose of this section is to recall the results of Ekedahl and Shepherd-Barron [ES04] and study the interplay between the conductrix and genus 1 fibrations. In particular, we will make extensive use of the tables in [ES04, Theorems 2.2 and 3.1]. Then, we will apply this knowledge to special extremal genus 1 fibrations. This will lead to the classification of the dual graphs of Enriques surfaces with finite automorphism group in the next section.

In this section, unless mentioned otherwise, all Enriques surfaces are classical or supersingular in characteristic 2 . For simplicity, we write $A_{1}$-singularity or $D_{4}$-singularity for a rational double point of type $A_{1}$ or of type $D_{4}$, respectively. Also, we will use the symbol $n A_{1}$ for $n$ rational double points of type $A_{1}$ (in characteristic 2 , there are two types, $D_{4}^{0}$ and $D_{4}^{1}$, of singularities with the same dual graph $D_{4}$ of exceptional curves. However, we need only the dual graph as information and do not keep track of the isomorphism class).

\subsection{Singularities of the canonical cover}

In [ES04], Ekedahl and Shepherd-Barron studied "exceptional" Enriques surfaces using the conductrix associated with their canonical cover. Recall the following structural result.

Proposition 3.1 (Ekedahl and Shepherd-Barron [ES04, Proposition 0.5]). Let $X$ be an Enriques surface and $A$ its conductrix. Assume $A \neq 0$. Then, $A$ is 1 -connected. Moreover, $A^{2}=-2, A$ is supported on (-2)-curves, and the normalization of the canonical cover has either four rational double points of type $A_{1}$ as singularities or one rational double point of type $D_{4}$.

The following lemma is the reason why there is a relation between the conductrix of an Enriques surface and singular fibers of its genus 1 fibrations.

Lemma 3.2 (Ekedahl and Shepherd-Barron [ES04, Lemma 0.9]). Let $X$ be an Enriques surface, $\rho: \tilde{X} \rightarrow X$ its canonical cover and $\pi: X \rightarrow \mathbf{P}^{1}$ a genus 1 fibration. Then the morphism $\rho$ factors through the pullback $X_{F}$ of $\pi$ by the Frobenius map on $\mathbf{P}^{1}$. The map $\tilde{X} \rightarrow X_{F}$ is an isomorphism outside of the double fibers of $\pi$.

Lemma 3.3. Let $X$ be an Enriques surface with conductrix $A$. Let $\pi$ be a genus 1 fibration on $X$.

(i) If $\pi$ is a quasi-elliptic fibration, then the curve of cusps of $\pi$ is a component of $A$ with multiplicity 1 and all other components of $A$ are contained in fibers of $\pi$.

(ii) If $\pi$ is an elliptic fibration, then $A$ is contained in one fiber of $\pi$.

In particular, $\pi$ is elliptic if and only if $A$ is contained in a fiber of $\pi$.

Proof. A non-zero regular 1-form $\eta$ on $X$ is given by the pullback of a regular 1-form on $\mathbf{P}^{1}$ (see [Kat82]). Assume that $\pi$ is quasi-elliptic. Let $F$ be a general cuspidal fiber and $t$ a local parameter at $\pi(F)$. Then, locally around the cusp, $F$ is given by the equation $\pi^{*} t=y^{2}+x^{3}$ (Bombieri-Mumford [BM76, Proposition 4]); hence, $\pi^{*}(d t)=x^{2} d x$, which vanishes twice at the cusp. Therefore, the curve of cusps is a component of $A$ with multiplicity 1. Similarly, for an arbitrary genus 1 fibration, one shows that $\eta$ does not vanish on any smooth point of a fiber of $\pi$. Since $A$ is connected by Proposition 3.1, this yields the second claim. 


\section{Classification of EnRiques surfaces}

Recall that the minimal dissolution of a double cover $Y \rightarrow X$ of surfaces with $X$ smooth and $Y$ normal is the successive blow-up of points on $X$ lying under singular points of $Y$. For an Enriques surface $X$, we call the minimal dissolution of the double cover $\tilde{X}_{\text {norm }} \rightarrow X$, where $\tilde{X}_{\text {norm }}$ is the normalization of the canonical cover $\tilde{X}$, the minimal dissolution of $X$ and denote it by $X_{\text {diss }}$. The normalization $\tilde{X}_{\mathrm{sm}}$ of $X_{\text {diss }}$ in $K(\tilde{X})$ is the minimal resolution of singularities of $\tilde{X}_{\text {norm }}$ if $\tilde{X}_{\text {norm }}$ has only rational singularities (which holds, for example, if $\tilde{X}_{\text {norm }} \neq \tilde{X}$ by Proposition 3.1), but it is not minimal in general. The following diagram, where the vertical arrows are finite morphisms of degree 2 and the horizontal arrows are birational morphisms, summarizes this discussion:

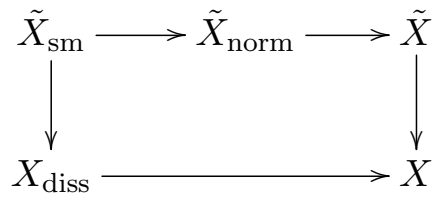

canonical cover

Enriques surface.

Now, we recall the results of Ekedahl and Shepherd-Barron [ES04] on what happens to (-2)curves on $X$ when we take their inverse image in $\tilde{X}_{\text {sm }}$ and additionally study curves of arithmetic genus 1 .

LEMMA 3.4. With the notation introduced above, let $C$ be an irreducible curve of arithmetic genus at most 1 on an Enriques surface $X$ with conductrix $A$. Denote the irreducible curve on $\tilde{X}_{\mathrm{sm}}$ mapping surjectively to $C$ by $\tilde{C}$, and let $\rho: \tilde{X}_{\mathrm{sm}} \rightarrow \tilde{X}$ and $\pi: \tilde{X} \rightarrow X$ be the morphisms from the normalization of the minimal dissolution of $X$ to $\tilde{X}$ and from $\tilde{X}$ to $X$, respectively. We fix the following invariants:

(i) the degree $s$ of $\left.(\pi \circ \rho)\right|_{\tilde{C}}: \tilde{C} \rightarrow C$

(ii) the number $r$ of points (possibly including infinitely near ones) on $C$ which are blown up during the minimal dissolution of $X$, and their multiplicity $m$

(iii) the intersection number $A \cdot C$

(iv) the self-intersection numbers $\tilde{C}^{2}$ and $C^{2}$

(v) the arithmetic genera $p_{a}(C)$ and $p_{a}(\tilde{C})$

(vi) if $p_{a}(C)=1$, the type Sing of singularity of $C$. (This is either nodal $\mathrm{n}$, cuspidal c or smooth sm.)

Then $\tilde{C}$ satisfies the following:

(a) $\tilde{C}^{2}=\left(C^{2}-m^{2} r\right) s^{2} / 2$ and $2 p_{a}(\tilde{C})-2=\tilde{C}^{2}-s A \cdot C$.

(b) If two curves meet transversally on $X$ and both have s-invariant 1 , then they do not meet on $X_{\text {diss }}$.

(c) For $A \cdot C \geqslant-2$ and $p_{a}(C)=0$, we have the following possibilities:

\begin{tabular}{ccccc}
$r$ & $s$ & $A \cdot C$ & $\tilde{C}^{2}$ & $p_{a}(\tilde{C})$ \\
\hline 0 & 1 & 1 & -1 & 0 \\
0 & 2 & -1 & -4 & 0 \\
2 & 1 & 0 & -2 & 0 \\
4 & 1 & -1 & -3 & 0 \\
6 & 1 & -2 & -4 & 0 \\
1 & 2 & -2 & -6 & 0
\end{tabular}




\section{T. Katsura, S. Kondō And G. Martin}

(d) For $p_{a}(C)=1$, we have the following possibilities:

\begin{tabular}{ccccccc} 
Sing & $r$ & $m$ & $s$ & $A \cdot C$ & $\tilde{C}^{2}$ & $p_{a}(\tilde{C})$ \\
\hline $\mathrm{sm}$ & 0 & & 1 & 0 & 0 & 1 \\
$\mathrm{sm}$ & 0 & & 2 & 0 & 0 & 1 \\
$\mathrm{n}$ & 1 & 2 & 1 & 0 & -2 & 0 \\
$\mathrm{c}$ & 0 & & 1 & 0 & 0 & 1 \\
$\mathrm{c}$ & 0 & & 2 & 0 & 0 & 1 \\
$\mathrm{c}$ & 1 & 2 & 1 & 0 & -2 & 0 \\
$\mathrm{c}$ & 4 & 1 & 1 & 0 & -2 & 0 \\
$\mathrm{c}$ & 2 & 1 & 1 & 1 & -1 & 0 \\
$\mathrm{c}$ & 0 & & 1 & 2 & 0 & 0
\end{tabular}

(e) Let $C$ be a cuspidal curve.

- If $|C|$ defines a quasi-elliptic fibration, then $r=0$ and $s=1$.

- If $|C|$ defines an elliptic fibration, then $r=1, m=2$ and $s=1$.

- If $|C|$ does not define a quasi-elliptic fibration and $|2 C|$ defines a quasi-elliptic fibration, then $r=2, m=1$ and $s=1$.

Proof. Similarly to what is done by Ekedahl and Shepherd-Barron [ES04], the formulas for the self-intersection number and the genus of $\tilde{C}$ are obtained by observing that the self-intersection number of $C$ drops by $m^{2}$ for every point of multiplicity $m$ on $C$ which is blown up during the minimal dissolution and from $\omega_{\tilde{X} / X}=\pi^{*}\left(\mathcal{O}_{X}(-A)\right)$. Also, claim (b) is in [ES04].

The first table is contained in [ES04]; we will only establish the second one. Therefore, assume $p_{a}(C)=1$. If $C$ is smooth, then $A \cdot C=0$ by Lemma 3.3, which leaves only the two possibilities listed. If $C$ has a node, then $|C|$ defines an elliptic fibration $\varphi$ with $C$ as a simple fiber. Therefore, formally locally around $C$, the surface $X$ is isomorphic to the Jacobian of $\varphi$, and by Lemma 3.2, we can find $\tilde{C}$ by doing Frobenius pullback along the base. But on an $\mathrm{I}_{1}$ fiber, an elliptic surface acquires an $A_{1}$-singularity at the singular point of the nodal curve after Frobenius pullback. Therefore, the node of $C$ is blown up during the minimal dissolution. A similar argument works if $C$ is cuspidal and $|C|$ defines an elliptic fibration.

If $C$ is cuspidal, we have enumerated all numerical possibilities except for the ones where $p_{a}(\tilde{C})=0$ and $s=2$. These cases do not occur. In fact, assume $s=2$ and $p_{a}(\tilde{C})=0$. Denote the image of $\tilde{C}$ on $\tilde{X}_{\text {norm }}$ by $\tilde{C}^{\prime}$. Since the singular point of $C$ is not blown up during the dissolution (by the self-intersection formula), we have $\tilde{C}^{\prime} \cong \tilde{C} \cong \mathbf{P}^{1}$. Then, the flat morphism $\varphi: \tilde{X}_{\text {norm }} \rightarrow X$ restricts to a morphism $\left.\varphi\right|_{\tilde{C}^{\prime}}: \tilde{C}^{\prime} \rightarrow C$. Since $s=2$, we have $\varphi^{*} C=\tilde{C}^{\prime}$, so $\left.\varphi\right|_{\tilde{C}^{\prime}}$ is nothing but the base change of $\varphi$ along the closed immersion $C \rightarrow X$ and as such it is a flat morphism. But a morphism from $\mathbf{P}^{1}$ to the cuspidal cubic is never flat.

For the last statement (e), observe that $|C|$ defines a quasi-elliptic fibration if and only if $A \cdot C=2$, and $|2 C|$ defines a quasi-elliptic fibration if and only if $A \cdot C=1$. This follows immediately from Lemma 3.3, which implies that $A \cdot C=D \cdot C$, where $D$ is the curve of cusps of $|C|$ (respectively, $|2 C|$ ).

Remark 3.5. Several of the numerical possibilities in Lemma 3.4 might be excluded by using Lang's list of possible configurations of singular fibers on rational elliptic surfaces in characteristic 2 (see [Lan00]) together with Lemma 3.2. However, we will not pursue this here. 


\section{Classification of EnRiques surfaces}

Lemma 3.6. Let $X$ be an Enriques surface with a quasi-elliptic fibration $\varphi$. Let $F$ be a fiber of $\varphi$. If $F$ is a double fiber, then two points on $F$ (possibly including infinitely near ones) are blown up during the minimal dissolution. If $F$ is simple, then no point on $F$ is blown up.

Proof. If $F$ is reducible, this can be read off from the table in [ES04, Theorem 2.2] since every $(-2)$-curve on a simple fiber has $r$-invariant 0 and exactly one $(-2)$-curve on a double fiber has $r$-invariant 2 while the others have $r$-invariant 0 . If $F$ is irreducible, this is the last statement of Lemma 3.4.

Corollary 3.7. Let $X$ be an Enriques surface with a quasi-elliptic fibration. Then the normalization $\tilde{X}_{\text {norm }}$ of the canonical cover has an isolated $D_{4}$-singularity if and only if $X$ is supersingular.

Proof. Let $\varphi$ be a quasi-elliptic fibration on $X$. Since the conductrix is non-empty by Lemma 3.3, the surface $\tilde{X}$ is not normal. Therefore, $\tilde{X}_{\text {norm }}$ has either four $A_{1}$ - or one $D_{4}$-singularity by Proposition 3.1. If $\varphi$ has two double fibers, at least two distinct points on $X$ are blown up during the minimal dissolution, by Lemma 3.6. In this case, $X$ is classical (Proposition 2.5) and $\tilde{X}$ has four $A_{1}$-singularities. If $\varphi$ has only one double fiber, at most two distinct points on $X$ are blown up. In this case, $X$ is supersingular and $\tilde{X}$ has one $D_{4}$-singularity.

\subsection{Special extremal genus 1 fibrations}

In this subsection, we present a detailed study of Enriques surfaces with special genus 1 fibrations, their conductrices and isolated singularities on their canonical cover. Throughout, we will use the observations summed up in the following lemma.

Lemma 3.8. Let $X$ be an Enriques surface with conductrix $A$, and let $\tilde{X}$ be its canonical cover. The following hold:

(i) If two (-2)-curves which meet transversally have s-invariant 1 , then their intersection is blown up.

(ii) Every (-2)-curve $C$ satisfies $C . A \leqslant 1$.

(iii) Every (-2)-curve which is not a component of the support of the conductrix has s-invariant 1.

Now let $\pi: X \rightarrow \mathbf{P}^{1}$ be a genus 1 pencil. Then the following hold:

(a) A singular fiber of type $\mathrm{I}_{n}$ of $\pi$ gives $n A_{1}$-singularities on $\tilde{X}$.

(b) If $A \neq \emptyset$ and $\pi$ has a singular fiber of type $\mathrm{I}_{n}$, then $\tilde{X}$ has four $A_{1}$-singularities.

(c) If $A \neq \emptyset$ and two disjoint (-2)-curves have positive $r$-invariant, then $\tilde{X}$ has four $A_{1}$ singularities.

(d) If $A \neq \emptyset$ and the sum of all $r$-invariants of fiber components is less than 4 , then $\tilde{X}$ has one $D_{4}$-singularity.

Proof. The first claim is obtained by checking intersection numbers, as was done by Ekedahl and Shepherd-Barron in [ES04, Definition-Lemma 0.8(iii)], and the second is immediate from Lemma 3.4(c). Since a curve $C$ which is not contained in $A$ has $A \cdot C \geqslant 0$, the third claim follows from Lemma 3.4.

For the statements about $\pi$ : By Lemma 3.2, the singularities lying over a simple fiber of $\pi$ can be read off from the Frobenius base change of the Jacobian fibration. Since an $\mathrm{I}_{n}$ fiber is simple and we can easily check (for example, using Tate's algorithm) that the Frobenius base 


\section{T. Katsura, S. Kondō And G. Martin}

change of a fiber of type $\mathrm{I}_{n}$ acquires an $A_{1}$ singularity at every point where two curves in the fiber meet, we obtain claim (a).

Claim (b) follows immediately from claim (a) since $\tilde{X}$ has either four $A_{1}$-singularities or one $D_{4}$-singularity if $A \neq \emptyset$ (see Proposition 3.1). Two disjoint curves having positive $r$-invariant means that distinct points are blown up during the dissolution, excluding the possibility of a $D_{4}$-singularity on the cover. Hence we obtain claim (c). For claim (d), note that the sum of the $r$-invariants of fiber components being less than 4 means that less than 4 distinct points are blown up, so the singularity can only be a $D_{4}$-singularity.

Remark 3.9. Observe that we have used that the singularities lying over a simple fiber of $\pi$ are the same as the singularities of the Frobenius base change of the Jacobian fibration over the corresponding fiber. This follows from Lemma 3.2 since in a formal neighborhood of a simple fiber, an elliptic fibration is isomorphic to its Jacobian.

Lemma 3.10. There are no special elliptic fibrations on Enriques surfaces with a double fiber of type $2 \mathrm{III}^{*}, 2 \mathrm{II}^{*}$ or $2 \mathrm{I}_{4}^{*}$. Moreover, if the conductrix is non-empty, a special elliptic fibration with a double fiber of type IV cannot exist.

Proof. The statement about II*, III* and I* is contained in Ekedahl and Shepherd-Barron [ES04, Corollary 3.2]. We will give another argument here. Let $N$ be a special 2 -section, and let $C$ be a simple component of the double fiber we want to exclude. Assume that $C$ meets $N$. By checking all possible conductrices $A$ of [ES04, Theorem 3.1], we obtain that $C$ and $N$ have $s$-invariant 1 . Moreover, $A \cdot C=0$ if $C$ is a component of $A$ with multiplicity 1 , whereas $A \cdot C=1$ if $C$ does not occur in the conductrix. Therefore, $N \cdot A=1$ if and only if $C \cdot A=0$. Now by Lemma 3.8(i), the intersection of $N$ and $C$ is blown up. But one of them has $r$-invariant 0 by Lemma 3.4(c). This gives a contradiction.

Now, we prove the second claim. Note that the fiber of type IV is disjoint from $A$ by [ES04, Theorem 3.1]. It follows from Lemma 3.3(ii) that the 2 -section $N$ is not contained in $A$. Hence $N$ has $s$-invariant 1 by Lemma 3.8(iii), and every component of the fiber of type IV also has $s$-invariant 1 by the same lemma. Therefore, the intersection of $N$ and the fiber of type IV is blown up during the dissolution (Lemma 3.8). Additionally, the intersection of the three components of the fiber of type IV is blown up. Therefore, the canonical cover has four $A_{1}$-singularities by Proposition 3.1 and all blow-ups happen on distinct point of the fiber of type IV. However, since at least one point of $N$ is blown up, $N$ has positive $r$-invariant and, by Lemma 3.3, we have that $N . A \geqslant 0$. Therefore, by Lemma 3.8(iii), the section $N$ has $r$-invariant 2 ; that is, two (possibly infinitely near) points on $N$ are blown up during the dissolution. Since $N$ meets the fiber of type IV only once, this is impossible.

Recall that Ekedahl and Shepherd-Barron gave a list of possibilities for the restriction of the conductrix $A$ to the fiber of any elliptic fibration [ES04, Theorem 3.1]. Their list contains not only the case $A^{2}=-2$ but also more general cases (for elliptic surfaces that are not necessarily Enriques surfaces). Moreover, in the case where $A^{2}=-2$, there are several possibilities for $A$ for a fixed elliptic fibration.

Remark 3.11. A priori, [ES04] gives the restriction of $A$ to a fiber $F$ only up to multiples of $F$ and up to multiples of the half-fiber underlying $F$ if $F$ is a double fiber. However, it is clear that the support of $A$ cannot contain $F$ since $h^{0}\left(X, \Omega_{X}^{1}\right)=1$, and the explicit classification of "exceptional Enriques surfaces" in [ES04] shows that in the cases where $A$ contains a half-fiber, 


\section{Classification of EnRiques surfaces}

$A$ is also in fact already contained in the tables of [ES04]. This implies that the tables in [ES04] do in fact give all possibilities for the restriction of $A$ to a fiber, not only up to multiples.

In Lemma 3.12 below, by using the list of [ES04, Theorem 3.1] and the above observations, we give the list of possible conductrices together with possible isolated singularities on the canonical cover for any extremal special elliptic fibration on an Enriques surface. In our case, it will turn out that the conductrix is in fact uniquely determined by the type of singular fibers. Tables 5 and 6 will enable us to set up a case-by-case analysis in the next section.

LEMMA 3.12. The isolated singularities on the normalization of the canonical cover of an Enriques surface with a special extremal elliptic fibration and the conductrix are summed up in Table 5. The self-intersection number of the reduced inverse image of a curve on the minimal resolution of singularities of the canonical cover is given as an index to the multiplicity.

Proof. For the list of rational extremal elliptic fibrations, see Proposition 2.7. Since the conduc$\operatorname{trix} A$ is contained in one fiber, the table in [ES04, Theorem 3.1] will give us the possibilities for $A$. In every case, we denote the special 2 -section by $N$. Recall that $A^{2}=-2$ by Proposition 3.1.

- $\left(\mathrm{I}_{4}^{*}\right)$ : There is only one possibility for $A$ with $A^{2}=-2$ in the list of [ES04]. The canonical cover has four $A_{1}$-singularities by Lemma 3.8(c).

- $\left(\mathrm{II}^{*}\right)$ : There are two possible conductrices with $A^{2}=-2$ in the list of [ES04]. However, since $N \cdot A \leqslant 1$ by Lemma 3.8(ii), we get the one in the table. Since all fibers different from the fiber of type $\mathrm{II}^{*}$ are smooth and no point on a smooth fiber is blown up during the dissolution, by Lemma 3.4, the sum of all $r$-invariants of fibers is less than 4 . Hence the cover has one $D_{4}$-singularity by Lemma $3.8(\mathrm{~d})$.

- (2III, $\left.\mathrm{I}_{8}\right)$ : In this case, $A=\emptyset$. Thus, any $(-2)$-curve has $s$-invariant 1 by Lemma 3.4(c). Since the intersection of $N$ with a component of the fiber of type III is blown up by Lemma 3.8(i), there are at least eleven distinct points which are blown up during the dissolution by Lemma 3.8(a). Therefore, the cover has twelve $A_{1}$-singularities.

- (III, $\left.\mathrm{I}_{8}\right)$ : Again, we have $A=\emptyset$. By [Lan00], the fiber of type III acquires a $D_{4}$-singularity after Frobenius pullback. The eight $A_{1}$-singularities come from the fiber of type $\mathrm{I}_{8}$ by Lemma 3.8(a).

- $\left(2 \mathrm{I}_{1}^{*}, \mathrm{I}_{4}\right)$ : In this case and the next, there are two possibilities for $A$ with $A^{2}=-2$ in the list of [ES04]. By Lemma 3.8(b), we have four $A_{1}$-singularities. Since $N$ is a 2 -section and every point which is blown up lies on the fiber of type $\mathrm{I}_{4}$, the $r$-invariant of $N$ is at most 1 and therefore $N \cdot A=1$, by Lemma 3.4(c). This is only possible for the conductrix in our table.

- $\left(\mathrm{I}_{1}^{*}, \mathrm{I}_{4}\right)$ : By the same argument as in the previous case, we have $N \cdot A=1$. Moreover, $N$ cannot meet distinct components of the fiber of type $I_{1}^{*}$ since we would obtain a different fibration with a double fiber of type $\mathrm{I}_{4}$ or $\mathrm{I}_{5}$ in these cases. Therefore, $N$ meets a multiplicity 2 component of the fiber of type $\mathrm{I}_{1}^{*}$. Now, $N$ and some components of the fiber of type $\mathrm{I}_{1}^{*}$ form a fiber of type $\mathrm{I}_{0}^{*}$ of a different fibration, and the only possible conductrix for this behavior is the one in our table.

- $\left(\mathrm{III}^{*}, \mathrm{I}_{2}\right)$ : There are two possible conductrices with $A^{2}=-2$ in the list of [ES04]. If the conductrix has the full fiber as support, $N$ meets the central multiplicity 2 component since $N \cdot A \leqslant 1$ by Lemma 3.8(ii). But then, there is a fiber of type $\mathrm{IV}^{*}$ of a different fibration such that two components of the conductrix meet the fiber without being contained in it. 
T. Katsura, S. Kondō And G. Martin

Table 5: Singularities on the canonical cover of an Enriques surface with a special and extremal elliptic fibration

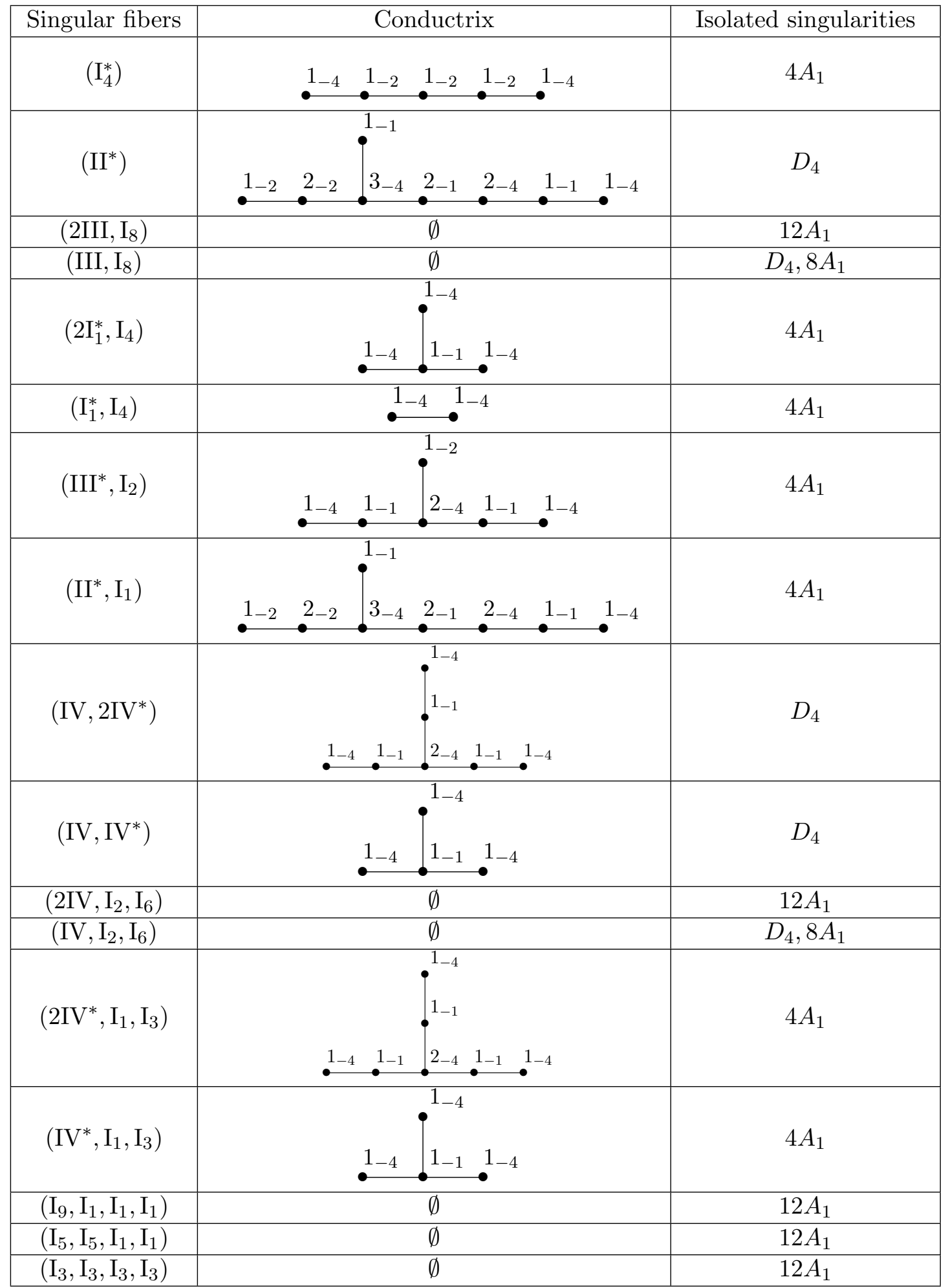




\section{Classification of EnRiques surfaces}

This is not possible, by Lemma 3.3. Hence we have the conductrix in our table and the isolated singularities because of Lemma 3.8(b).

- $\left(\mathrm{II}^{*}, \mathrm{I}_{1}\right)$ : The conductrix is the one in the table by the same argument as in case $\left(\mathrm{II}^{*}\right)$. By Lemma 3.8(b), we get the types of isolated singularities.

- $\left(\mathrm{IV}, 2 \mathrm{IV}^{*}\right)$ : In this case and the next, there are two possibilities for $A$ with $A^{2}=-2$ in the list of [ES04]. Since $N$ meets a simple component of the fiber of type $\mathrm{IV}^{*}$, we can exclude the case where the conductrix does not have the full fiber as support, since in this case every simple component of the fiber of type $\mathrm{IV}^{*}$ has $s$-invariant 1 and $r$-invariant 0 while $N$ has $s$-invariant 1 by $N \cdot A=0$, contradicting Lemma 3.8(i). The isolated singularities are as in the table since by [Lan00] the fibers of type IV acquires a $D_{4}$-singularity after Frobenius pullback.

- $\left(\mathrm{IV}, \mathrm{IV}^{*}\right)$ : Suppose that $A$ has the full fiber of type $\mathrm{IV}^{*}$ as support. Then $N$ meets a multiplicity 2 component of this fiber since $A \cdot N \leqslant 1$. But then $N$ and components of the fiber of type $\mathrm{IV}^{*}$ form a fiber of type $\mathrm{I}_{1}^{*}$ of a different elliptic fibration such that two components of the conductrix meet the fiber without being contained in it. This is not possible, by Lemma 3.3. As in the previous case, we get a $D_{4}$-singularity.

- $\left(2 \mathrm{IV}, \mathrm{I}_{2}, \mathrm{I}_{6}\right)$ and $\left(\mathrm{IV}, \mathrm{I}_{2}, \mathrm{I}_{6}\right)$ : The argument is essentially the same as in the cases $\left(2 \mathrm{III}, \mathrm{I}_{8}\right)$ and (III, $\left.\mathrm{I}_{8}\right)$.

- $\left(2 \mathrm{IV}^{*}, \mathrm{I}_{1}, \mathrm{I}_{3}\right)$ and $\left(\mathrm{IV}^{*}, \mathrm{I}_{1}, \mathrm{I}_{3}\right)$ : The argument is similar to the cases with singular fibers $\left(\mathrm{IV}, 2 \mathrm{IV}^{*}\right)$ and $\left(\mathrm{IV}, \mathrm{IV}^{*}\right)$, except that the fibers of type $\mathrm{I}_{n}$ give four $A_{1}$-singularities, by Lemma 3.8(a).

- All singular fibers multiplicative: In these cases, we get twelve $A_{1}$-singularities by Lemma 3.8(a).

For the convenience of the reader, we give the corresponding table for quasi-elliptic fibrations. This does not require proof since the conductrices are uniquely determined [ES04] and the isolated singularities depend on the number of double fibers (see Lemma 3.6). Since fibers of type III do not contribute to the conductrix, we will not specify their multiplicity. Also, recall that by Lemma 3.3, the curve of cusps is the only component of the conductrix which is not contained in fibers of the fibration.

LEMMA 3.13. The isolated singularities on the normalization of the canonical cover of an Enriques surface with a quasi-elliptic fibration and the conductrix are summed up in Table 6. The selfintersection number of the reduced inverse image of the curve on the minimal resolution of singularities of the canonical cover is given as an index to the multiplicity. We do not give multiplicities of the fibers of type III. The curve of cusps is encircled.

Remark 3.14. Recall that any Enriques surface has a genus 1 fibration (Proposition 2.4) and that if an Enriques surface $X$ has a finite group of automorphisms, then any genus 1 fibration on $X$ is extremal (Proposition 2.10). Therefore, $X$ has an extremal special genus 1 fibration by Proposition 2.11. Lemmas 3.12 and 3.13 imply that the canonical cover of any Enriques surface with finite automorphism group has only $A_{1^{-}}$or $D_{4}$-singularities as isolated singularities. In particular, this excludes the exotic case where the cover has an elliptic singularity; we refer the reader to [Sch17, Mat18] for an overview of the types of singularities that can occur in general. 
T. Katsura, S. Kondō And G. Martin

Table 6: Singularities on the canonical cover of an Enriques surface with a quasi-elliptic fibration

\begin{tabular}{|c|c|c|}
\hline Singular fibers & Conductrix & Isolated singularities \\
\hline$\left(2 \mathrm{II}^{*}\right)$ & 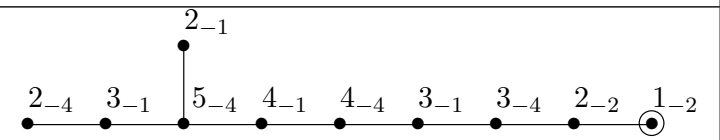 & $4 A_{1}$ or $D_{4}$ \\
\hline$\left(\mathrm{II}^{*}\right)$ & 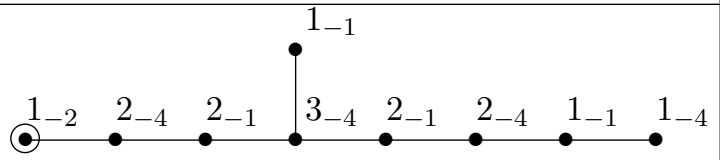 & $4 A_{1}$ or $D_{4}$ \\
\hline$\left(2 \mathrm{I}_{4}^{*}\right)$ & 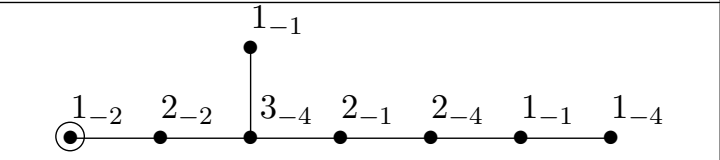 & $4 A_{1}$ or $D_{4}$ \\
\hline$\left(\mathrm{I}_{4}^{*}\right)$ & $1_{-4} 1_{-1} \oint^{1-2} 2_{-4} 1_{-1} 1_{-4}$ & $4 A_{1}$ or $D_{4}$ \\
\hline$\left(2 \mathrm{III}{ }^{*}, \mathrm{III}\right)$ & 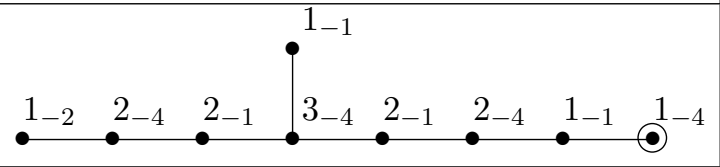 & $4 A_{1}$ or $D_{4}$ \\
\hline$\left(\mathrm{III}^{*}, \mathrm{III}\right)$ & 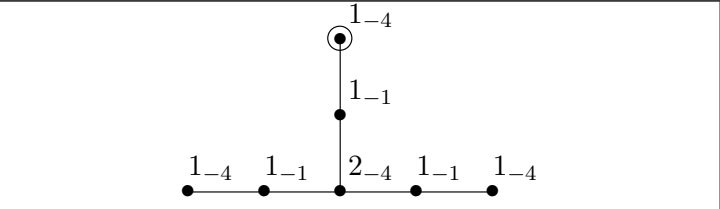 & $4 A_{1}$ or $D_{4}$ \\
\hline$\left(2 \mathrm{I}_{0}^{*}, 2 \mathrm{I}_{0}^{*}\right)$ & $\bullet^{1_{-4}} \bullet^{1_{-2}} \bullet^{1_{-2}} \bullet^{1_{-2}} \bullet^{1_{-4}}$ & $4 A_{1}$ \\
\hline$\left(2 \mathrm{I}_{0}^{*}, \mathrm{I}_{0}^{*}\right)$ & $\bullet^{1-4} \bigodot^{1-2} \bullet^{1_{-2}} \bullet^{1_{-4}}$ & $4 A_{1}$ or $D_{4}$ \\
\hline$\left(\mathrm{I}_{0}^{*}, \mathrm{I}_{0}^{*}\right)$ & $\bullet_{-4}^{1_{-2}^{1-2}} \bullet^{1_{-4}}$ & $4 A_{1}$ or $D_{4}$ \\
\hline$\left(2 \mathrm{I}_{2}^{*}, \mathrm{III}, \mathrm{III}\right)$ & $\bullet^{1-4} \bullet^{1_{-1}} \bullet^{1_{-2}} 2^{2} \bullet^{1_{-1}} \bullet^{1_{-4}}$ & $4 A_{1}$ or $D_{4}$ \\
\hline$\left(\mathrm{I}_{2}^{*}, \mathrm{III}, \mathrm{III}\right)$ & 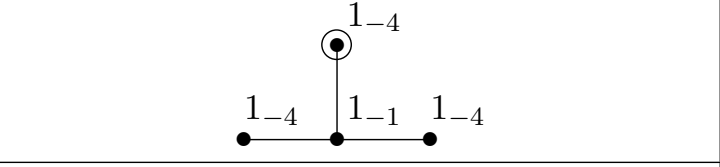 & $4 A_{1}$ or $D_{4}$ \\
\hline$\left(2 \mathrm{I}_{0}^{*}, 4 \times \mathrm{III}\right)$ & $\overbrace{}^{1-4} \bullet^{1_{-2}} \bullet^{1_{-4}}$ & $4 A_{1}$ or $D_{4}$ \\
\hline$\left(\mathrm{I}_{0}^{*}, 4 \times \mathrm{III}\right)$ & $\overbrace{}^{1-4} \bullet^{1-4}$ & $4 A_{1}$ or $D_{4}$ \\
\hline$(8 \times \mathrm{III})$ & $\odot^{1-6}$ & $4 A_{1}$ or $D_{4}$ \\
\hline
\end{tabular}




\section{Classification of EnRiques surfaces}

\section{Possible dual graphs}

THEOREM 4.1. Assume that $X$ is a classical or supersingular Enriques surface in characteristic 2 with a finite group of automorphisms. Then, the dual graph of $(-2)$-curves on $X$ is one of the dual graphs given in Theorems 1.3(a) and 1.4(a).

Proof. Recall that there exists a special genus 1 fibration on $X$ (Proposition 2.11) which is extremal (Proposition 2.10). Hence the pair $(A, I)$, where $A$ is the conductrix of $X$ and $I$ is the set of isolated singularities of the normalization of the canonical cover of $X$, is contained in Table 5 or 6 .

Using Lemmas 3.12 and 3.13 and the fact that every genus 1 fibration on $X$ is extremal by Proposition 2.10, we obtain a list of all possible special genus 1 fibration on $X$ depending on $(A, I)$. On the other hand, by Proposition 2.4, every configuration of $(-2)$-curves whose dual graph is an extended Dynkin diagram is in fact a fiber of a genus 1 fibration of $X$. These two observations together will allow us to control the $(-2)$-curves on $X$. It remains to go through the possibilities for $(A, I)$ and, starting with one of the possible special extremal fibrations, to show that either we arrive at a contradiction or $X$ contains one of the dual graphs of $(-2)$-curves in our list. In the latter case, Vinberg's criterion (Propositions 2.14 and 2.16 and Remark 2.15) shows immediately that these are in fact all $(-2)$-curves on $X$. For the fibrations that actually occur on Enriques surfaces with finite automorphism group, we refer the reader to the list of the genus 1 fibrations in the appendix.

We will make extensive use of Lemma 3.3 together with Tables 5 and 6 and the tables in [ES04, Theorems 2.2 and 3.1], which tell us how reducible fibers of genus 1 fibrations can meet $A$. We will give details on how to use these results in the first few cases, so that the reader can become familiar with the techniques and fill in the details of the later cases in a similar way. Also, we denote by $N$ a special (-2)-section for a given special genus 1 fibration. If the fibration is quasi-elliptic, then $N$ denotes the curve of cusps.

(1) Conductrix:

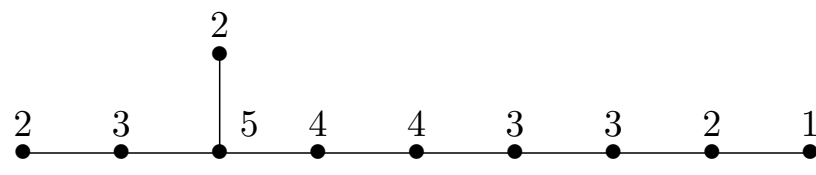

Singularities: $D_{4}$ or $4 A_{1}$

Possible special extremal fibrations: $\left(2 \mathrm{II}^{*}\right)$ quasi-elliptic

This is nothing but the dual graph of $(-2)$-curves of Enriques surfaces of type $\tilde{E}_{8}$. The Enriques surfaces are supersingular or classical according to the type of singularities (Corollary 3.7). These are the $\tilde{E}_{8}$-exceptional surfaces studied in [ES04].

(2) Conductrix:

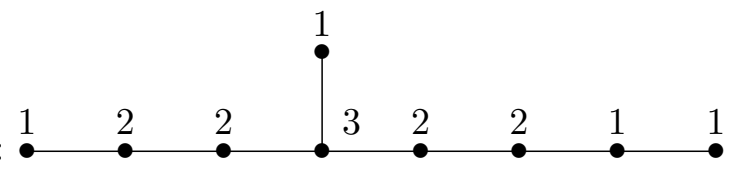
Singularities: $D_{4}$ or $4 A_{1}$ Possible special extremal fibrations: (II*) quasi-elliptic, (2III*, III) quasi-elliptic, and (2III* ,2III) quasi-elliptic

In this case, we will show that $X$ has the dual graph of an Enriques surface of type $\tilde{E}_{7}+\tilde{A}_{1}{ }^{(1)}$ or $\tilde{E}_{7}+\tilde{A}_{1}^{(2)}$.

First, note that if $X$ admits a quasi-elliptic fibration of type (2III*, III), the support of $A$ is exactly the support of the III* fiber and the curve of cusps $N$, by Table 6 and Lemma 3.3. 


\section{T. Katsura, S. Kondō And G. Martin}

More precisely, the rightmost vertex of $A$ corresponds to $N$. The 2 -section $N$ meets each component of the singular fiber of type III because otherwise there would be a (-2)-curve in the fiber of type III meeting $N$ (the conductrix) more than once, contradicting Lemma 3.8(ii). Now, fiber components and the special 2-section $N$ of the fibration form the dual graph of type $\tilde{E}_{7}+\tilde{A}_{1}^{(1)}$.

If $X$ admits a quasi-elliptic fibration of type (II*) or of type $\left(2 \mathrm{III}^{*}, 2 \mathrm{III}\right)$, then we im-

mediately get the dual graph of type $\tilde{E}_{7}+\tilde{A}_{1}^{(2)}$. These are the $\tilde{E}_{7}$ exceptional surfaces of [ES04].

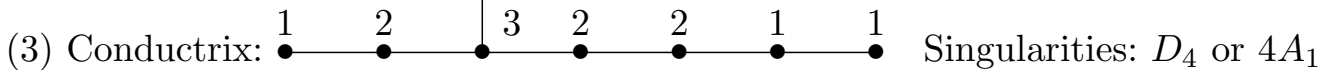

Possible special extremal fibrations: $\left(2 \mathrm{I}_{4}^{*}\right)$ quasi-elliptic, $\left(\mathrm{II}^{*}\right)$ elliptic and $\left(\mathrm{II}^{*}, \mathrm{I}_{1}\right)$ elliptic

In this case, we will show that $X$ has the dual graph of an Enriques surface of type $\tilde{D}_{8}$. Starting from the quasi-elliptic fibration of type $\left(2 \mathrm{I}_{4}^{*}\right)$, we immediately obtain the dual graph of Enriques surfaces of type $\tilde{D}_{8}$.

If we start with a special elliptic fibration with a singular fiber of type II* $^{*}$, the 2-section $N$ has to meet this fiber in a component with multiplicity 2 . Indeed, otherwise $N$ would meet the simple component $E_{1}$ of the fiber of type II $^{*}$ twice, and by Table 5 and Lemma 3.3, the component $E_{1}$ is not contained in $A$ while the adjacent component $E_{2}$ of the II*-fiber is contained in $A$. By Proposition 2.4 , and since $\left(E_{1}+N\right) \cdot E_{2}=1$ prevents $\left|E_{1}+N\right|$ from inducing a fibration, $\left|2\left(E_{1}+N\right)\right|$ would induce a special genus 1 fibration with special 2-section $E_{2}$, and $E_{1}$ and $N$ form a fiber of type 2III. However, such a fibration cannot occur on $X$ by the above list of possible special extremal fibrations.

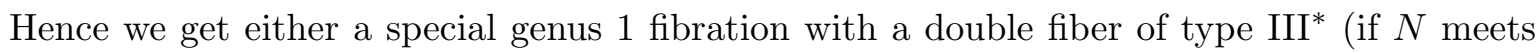
the leftmost vertex of $A$; then the rightmost vertex is a 2 -section) or a special genus 1 fibration with a double fiber of type $\mathrm{I}_{4}^{*}$ (if $N$ meets the rightmost vertex of $A$; then, the leftmost vertex is a 2-section). The first case is not allowed (it does not appear in the above list of possible special extremal fibrations). Therefore, we arrive at the dual graph of an Enriques surface of type $\tilde{D}_{8}$.

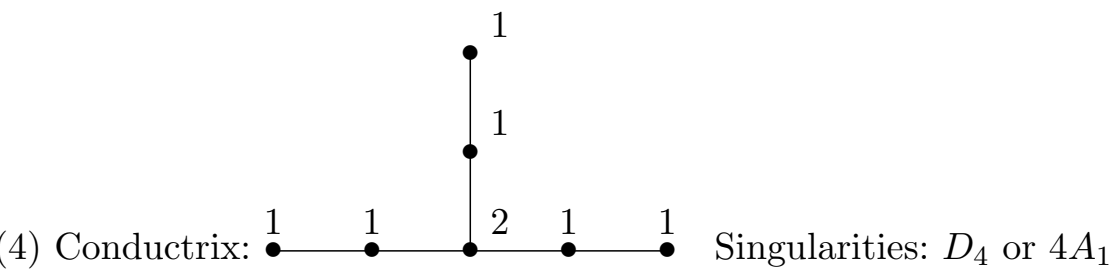

Possible special extremal fibrations: (III*, 2III) quasi-elliptic, (III*, III) quasi-elliptic, (2IV*, IV) elliptic and $\left(2 \mathrm{IV}^{*}, \mathrm{I}_{3}, \mathrm{I}_{1}\right)$ elliptic

In this case, we will show that $X$ has the dual graph of an Enriques surface of type $\tilde{E}_{6}+\tilde{A}_{2}$.

If we start with $\left(2 \mathrm{IV}^{*}, \mathrm{IV}\right)$ or $\left(2 \mathrm{IV}^{*}, \mathrm{I}_{3}, \mathrm{I}_{1}\right)$, then $N$ meets a component $E$ of the IV- or $\mathrm{I}_{3}$ fiber twice. Indeed, otherwise we would find a special genus 1 fibration with a double fiber of type IV or of type $\mathrm{I}_{3}$ consisting of $N$ and two components of the fiber of type IV or $\mathrm{I}_{3}$, which is not allowed in the above list of special fibrations. Then, using our list of possible fibrations, we see that $|2(E+N)|$ induces a quasi-elliptic fibration of type (III*,2III). We add the two 


\section{Classification of EnRiques surfaces}

simple components of the III*-fiber to the above diagram and compute the intersection numbers by repeating the above argument that a special 2 -section of the fibration of type $\left(2 \mathrm{IV}^{*}, \mathrm{IV}\right)$ or $\left(2 \mathrm{IV}^{*}, \mathrm{I}_{3}, \mathrm{I}_{1}\right)$ meets a component of the reducible simple fiber twice. Finally, we arrive at the dual graph of Enriques surfaces of type $\tilde{E}_{6}+\tilde{A}_{2}$. This is an $\tilde{E}_{6}$-exceptional Enriques surface of [ES04].

If $X$ has a quasi-elliptic fibration with a singular fiber of type III*, we will show that $X$ admits a special elliptic fibration with a singular fiber of type $2 \mathrm{IV}^{*}$, returning us to the argument of the previous paragraph. In this case, Lemma 3.3 and Table 6 show that the curve of cusps $N$ meets the component on the short tail of the III*-fiber. Thus, $N$ and components of the III*-fiber form a configuration of type $\tilde{E}_{6}$. By Proposition 2.4, and since the simple components of the original fiber of type III $^{*}$ are 2-sections, this configuration is the support of a fiber of a special genus 1 fibration on $X$. Using our list, we see that it is of type $\left(2 \mathrm{IV}^{*}, \mathrm{IV}\right)$ or $\left(2 \mathrm{IV}^{*}, \mathrm{I}_{3}, \mathrm{I}_{1}\right)$.

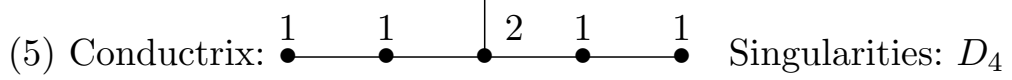

Possible special extremal fibrations: (I $\left.\mathrm{I}_{4}^{*}\right)$ quasi-elliptic, (2I2, III, III) quasi-elliptic and $\left(2 \mathrm{I}_{2}^{*}, 2 \mathrm{III}, \mathrm{III}\right)$ quasi-elliptic

We will show that this case does not occur on an Enriques surface with finite automorphism group.

In cases $\left(2 \mathrm{I}_{2}^{*}, \mathrm{III}, \mathrm{III}\right)$ and $\left(2 \mathrm{I}_{2}^{*}, 2 \mathrm{III}, \mathrm{III}\right)$, the curve of cusps $N$ corresponds to the leftmost (or rightmost) vertex of $A$ by Table 6 . Therefore, $N$ meets both components of a simple III fiber once by Lemma 3.8(ii); hence, there exists a genus 1 fibration with a fiber of type III* (here we use Proposition 2.4). Since the fiber of type III* contains the conductrix, the induced fibration is elliptic by Lemma 3.3 and hence of type $\left(\mathrm{III}^{*}, \mathrm{I}_{2}\right)$ by Proposition 2.7. This contradicts the type of singularities (Lemma 3.8(b)).

If we start with $\left(\mathrm{I}_{4}^{*}\right)$, then we find a special fibration with a double fiber of type $\mathrm{I}_{2}^{*}$ (again, use Table 6, Lemma 3.3, and a component of $\mathrm{I}_{4}^{*}$ as 2 -section). Thus, we reduce this case to the case of a quasi-elliptic fibration with a singular fiber of type $2 \mathrm{I}_{2}^{*}$, which we have excluded above.

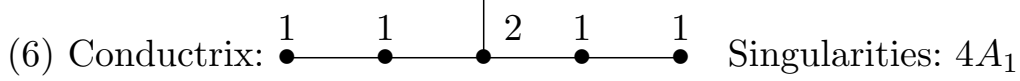

Possible special extremal fibrations: (2I $\left.{ }_{2}^{*}, 2 \mathrm{III}, \mathrm{III}\right)$ quasi-elliptic, (2I $\mathrm{I}_{2}^{*}$, III, III) quasi-elliptic, $\left(\mathrm{I}_{4}^{*}\right)$ quasi-elliptic and $\left(\mathrm{III}^{*}, \mathrm{I}_{2}\right)$ elliptic

We will prove that this case does not occur on an Enriques surface with finite automorphism group.

First, we show that in every case, there is a quasi-elliptic fibration with a singular fiber of type $I_{4}^{*}$ and with the curve of cusps meeting the central component.

In the cases with a double fiber of type $I_{2}^{*}$, we may assume that the curve of cusps is the leftmost vertex of $A$ by Table 6 . We observe that the curve of cusps cannot meet a component of a simple fiber of type III twice, because of Lemma 3.8(ii). Hence we obtain a quasi-elliptic fibration with a singular fiber of type $I_{4}^{*}$. 


\section{T. Katsura, S. Kondō And G. Martin}

In the case of the special elliptic fibration with singular fibers of type (III*, $\mathrm{I}_{2}$ ), note that if the 2-section meets a simple component of the fiber of type III* twice or meets two simple components, we get a quasi-elliptic fibration with a singular fiber of type $2 \mathrm{III}$ or $2 \mathrm{I}_{8}$. The latter case is impossible. Thus, we get a quasi-elliptic fibration with singular fibers of type $\left(2 \mathrm{I}_{2}^{*}, 2 \mathrm{III}, \mathrm{III}\right)$ by the above list of possible special extremal fibrations, and hence reduce this case to the previous one. If the 2-section meets a component of multiplicity 2 on one of the long tails, we get a quasielliptic fibration with a singular fiber of type $2 \mathrm{I}_{2}^{*}$, and if it meets the component of multiplicity 2 on the short tail, there would be a special elliptic fibration with a double fiber of type IV*. The last case does not occur by the list of possible special extremal fibrations.

We now start from a quasi-elliptic fibration with a singular fiber of type $I_{4}^{*}$ and exclude this case. By Table 6, the curve of cusps $N$ (which is denoted by the encircled vertex) meets the central component of the $\mathrm{I}_{4}^{*}$-fiber. Two of the blown-up points lie on the conductrix and two do not (by Table 6 and Lemma 3.4(c)). Any (-2)-curve not meeting the conductrix has $r$-invariant 2, and therefore it passes through the two blown-up points not lying on the conductrix. In particular, any two $(-2)$-curves not meeting the conductrix meet each other at least twice.

The configuration we start with is the following:

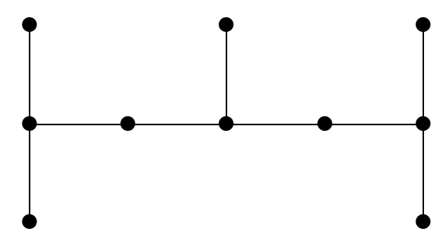

There are four subdiagrams of type $\tilde{E}_{7}$. If the automorphism group of an Enriques surface with this conductrix is finite, the elliptic fibrations induced by those subdiagrams have singular fibers of type $\left(\mathrm{III}^{*}, \mathrm{I}_{2}\right)$ or $\left(2 \mathrm{III}^{*}, \mathrm{I}_{2}\right)$. For any of these diagrams of type $\tilde{E}_{7}$, the two remaining curves are either 4 - or 2-sections of the fibration, depending on whether the fiber of type III* $^{*}$ is double or not (we do not know whether the fibration is special or not if the III*-fiber is a double fiber, and hence it might not be in our list). If such a multisection meets a component of the fiber of type $\mathrm{I}_{2}$ only once, we obtain a quasi-elliptic fibration with singular fiber of type $\mathrm{II}^{*}$ (since the conductrix $A$ is not contained in the fiber of type $\mathrm{II}^{*}$, the fibration is quasi-elliptic (Lemma 3.3(ii)) and hence special (Remark 2.12)), which is not allowed by our list. Hence the intersection number of each of the multisections with a component of the fiber of type $\mathrm{I}_{2}$ is 0,2 or 4 . If one of the multisections meets only one component of the fiber of type $\mathrm{I}_{2}$, the other multisection and the other component of the fiber of type $\mathrm{I}_{2}$ are disjoint from a diagram of type $\tilde{D}_{6}+A_{1}$; hence, they meet each other twice. This yields the dual graph (a) below, in which the new vertex is the other component as above. The dual graph (b) occurs if both multisections meet both components of the fiber of type $\mathrm{I}_{2}$ (this means that the multisections are 4-sections).

(a)

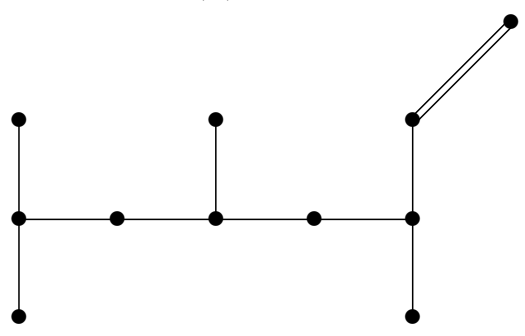

(b)

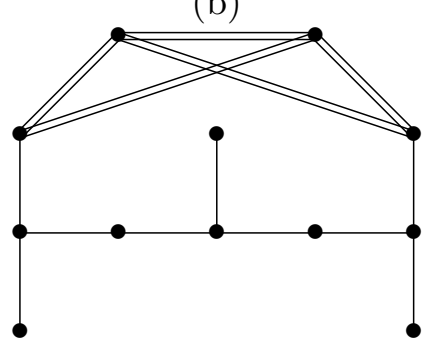

(i) We first exclude case (b). Using one of the diagrams of type $\tilde{A}_{1}$ which yields a quasi-elliptic 


\section{Classification of Enriques surfaces}

fibration with singular fibers $\left(2 \mathrm{I}_{2}^{*}, 2 \mathrm{III}, \mathrm{III}\right)$, we get the graph

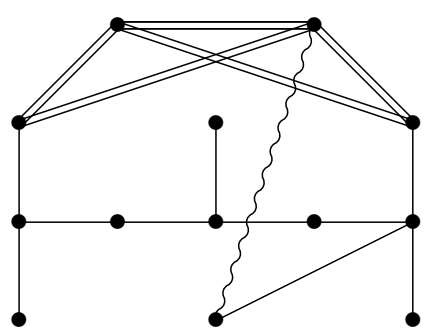

where the new vertex corresponds to a simple component of the fiber of type $I_{2}^{*}$ and where a wiggly line means that the intersection number of the adjacent vertices is 4 . Therefore, there is a subdiagram of type $\tilde{D}_{4}$ which defines a quasi-elliptic fibration with a double fiber of type $\mathrm{I}_{0}^{*}$ (by Proposition 2.4). This is not allowed by our list of special extremal fibrations.

(ii) Now we exclude case (a). Since case (b) does not occur, the other three $\tilde{E}_{7}$ diagrams give rise to (-2)-curves as in case (a), and, in particular, we get the graph

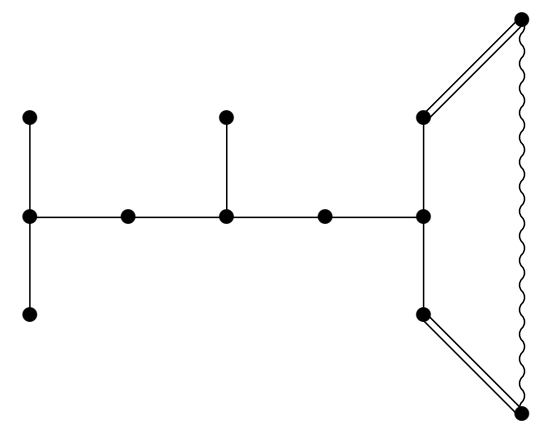

Here, the wiggly line denotes a non-negative intersection number. The $\tilde{D}_{6}$ diagram on the left gives rise to a quasi-elliptic fibration. Since the two $\tilde{A}_{1}$ diagrams plus the wiggly line are perpendicular to the $\tilde{D}_{6}$ diagram, they are supported on fibers of this fibration, which is only possible if the wiggly line denotes the intersection number zero. However, this implies that the fibration has singular fibers of type $\left(2 \mathrm{I}_{2}^{*}, 2 \mathrm{III}^{*}, 2 \mathrm{III}^{*}\right)$, which gives a contradiction. Therefore, an Enriques surface with finite automorphism group and this conductrix cannot exist.

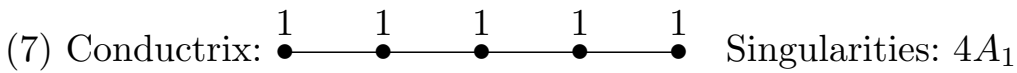

Possible special extremal fibrations: $\left(2 \mathrm{I}_{0}^{*}, 2 \mathrm{I}_{0}^{*}\right)$ quasi-elliptic and $\left(\mathrm{I}_{4}^{*}\right)$ elliptic

In this case, we will show that $X$ has the dual graph of an Enriques surface of type $\tilde{D}_{4}+\tilde{D}_{4}$. If we start with a special elliptic fibration with a singular fiber of type $\left(2 \mathrm{I}_{0}^{*}, 2 \mathrm{I}_{0}^{*}\right)$, then we immediately obtain the dual graph of Enriques surface of type $\tilde{D}_{4}+\tilde{D}_{4}$.

In the case of a special elliptic fibration with a singular fiber of type $\left(\mathrm{I}_{4}^{*}\right)$, we will reduce to the previous paragraph. First, note that the support of $A$ is equal to the support of the components of $\mathrm{I}_{4}^{*}$ of multiplicity 2 by [ES04, Theorem 3.1]. Now, we have to observe that a special 2-section $N$ has to meet the conductrix, for otherwise we would obtain a special genus 1 fibration with a singular fiber of type 2III, $\mathrm{I}_{4}$ or $\mathrm{I}_{8}$, which is not allowed by the above list of special extremal fibrations. If the 2-section $N$ meets the conductrix, we obtain a special genus 1 fibration with a singular double fiber of type $\mathrm{I}_{2}^{*}, \mathrm{I}_{1}^{*}$ or $\mathrm{I}_{0}^{*}$. The first two are not allowed by the above list. Thus, we get a quasi-elliptic fibration with a double fiber of type $\mathrm{I}_{0}^{*}$. 


\section{T. Katsura, S. Kondō And G. Martin}

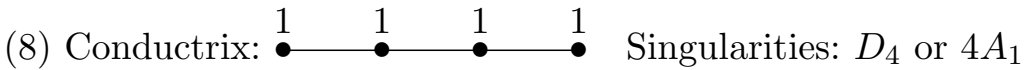

Possible special extremal fibrations: $\left(2 \mathrm{I}_{0}^{*}, \mathrm{I}_{0}^{*}\right)$ quasi-elliptic

We will show that this case does not occur on an Enriques surface with finite automorphism group. Starting with a fibration with singular fibers of type $\left(2 \mathrm{I}_{0}^{*}, \mathrm{I}_{0}^{*}\right)$, the curve of cusps $N$ meets the component with multiplicity 2 of the singular fiber of type $\mathrm{I}_{0}^{*}$ (use Table 6 and [ES04, Theorem 2.2]), and hence there is a subdiagram of type $\tilde{D}_{7}$ which defines a non-extremal fibration (Propositions 2.7 and 2.8). Therefore, an Enriques surface with this conductrix cannot have a finite automorphism group.

(9) Conductrix: $\stackrel{1}{\bullet} \quad 1 \quad$ Singularities: $D_{4}$ or $4 A_{1}$

Possible special extremal fibrations: $\left(\mathrm{I}_{0}^{*}, \mathrm{I}_{0}^{*}\right)$ quasi-elliptic, $\left(2 \mathrm{I}_{0}^{*}, 2 \mathrm{III}, \mathrm{III}, \mathrm{III}, \mathrm{III}\right)$ quasi-elliptic and $\left(2 I_{0}^{*}\right.$, III, III, III, III) quasi-elliptic

We will show that this case does not occur on an Enriques surface with finite automorphism group. Starting with a quasi-elliptic fibration with singular fibers of type $\left(\mathrm{I}_{0}^{*}, \mathrm{I}_{0}^{*}\right)$, we obtain an elliptic fibration with a singular fiber of type $\mathrm{I}_{2}^{*}$ (again use Table 6 and [ES04, Theorem 2.2]), which is not extremal by Proposition 2.7.

As for the fibrations with a double fiber of type $2 \mathrm{I}_{0}^{*}$, by Lemma 3.8(ii), the curve of cusps $N$ meets two components of each simple fiber of type III. Therefore, there is a diagram of type $\tilde{D}_{6}$ containing the conductrix. By Lemma 3.3, the corresponding fibration is elliptic. But an elliptic fibration with a fiber of type $I_{2}^{*}$ cannot be extremal by Proposition 2.7.

(10) Conductrix: $\stackrel{1}{\bullet} \quad$ Singularities: $D_{4}$ or $4 A_{1}$

Possible special extremal fibrations: (I $\mathrm{I}_{0}^{*}$, III, III, III, III) quasi-elliptic, (I $\mathrm{I}_{0}^{*}, 2 \mathrm{III}$, III, III, III) quasielliptic, ( $\left.\mathrm{I}_{0}^{*}, 2 \mathrm{III}, 2 \mathrm{III}, \mathrm{III}, \mathrm{III}\right)$ quasi-elliptic and $\left(\mathrm{I}_{1}^{*}, \mathrm{I}_{4}\right)$ elliptic.

We will show that this case does not occur on an Enriques surface with finite automorphism group. If there is a quasi-elliptic fibration on this surface (that is, in one of the first three cases in the above list), we see that there is a configuration of type $\tilde{D}_{4}$ containing the conductrix by using Table 6 and [ES04, Theorem 2.2]. It defines an elliptic fibration (Proposition 2.4) which is not extremal by Proposition 2.7.

Starting with a special elliptic fibration with singular fibers of type $\left(\mathrm{I}_{1}^{*}, \mathrm{I}_{4}\right)$, we look at the intersection of $N$ with the fiber of type $I_{1}^{*}$. Using Table 5 and [ES04, Theorem 3.1], we can see that the conductrix consists of the two components of the fiber of type $\mathrm{I}_{1}^{*}$ with multiplicity 2 . If the special 2-section $N$ meets distinct components, we obtain a configuration giving a double fiber of type $\mathrm{I}_{4}$ or $\mathrm{I}_{5}$, which gives a contradiction. If $N$ meets a double component once, then there is a special fibration with a fiber of type $\mathrm{I}_{0}^{*}$ containing the conductrix. Such a fibration is not contained in our list. If $N$ meets a simple component twice, we get a double fiber of type III of a quasi-elliptic fibration. Thus, we have reduced this case to the quasi-elliptic case.

(11) Conductrix: $1 \quad$ Singularities: $D_{4}$ or $4 A_{1}$

Possible special extremal fibrations: (III, III, III, III, III, III, III, III) quasi-elliptic, any multiplicities

We will show that this case does not occur on an Enriques surface with finite automorphism group. The 2-section $N$ is nothing but the conductrix, and hence $N$ meets two components of 


\section{Classification of EnRiques surfaces}

each simple fiber of type III by Lemma 3.8(ii). Thus, we have an elliptic fibration with a fiber of type $\mathrm{I}_{0}^{*}$ which is not extremal by Proposition 2.7 .

(12) Conductrix: $\stackrel{\iota^{1}}{\bullet} \stackrel{1}{\bullet}$ Singularities: $4 A_{1}$

Possible special extremal fibrations: (I $\left.\mathrm{I}_{2}^{*}, 2 \mathrm{III}, 2 \mathrm{III}\right)$ quasi-elliptic, (I $\mathrm{I}_{2}^{*}$, III, 2III) quasi-elliptic,

$\left(\mathrm{I}_{2}^{*}, \mathrm{III}, \mathrm{III}\right)$ quasi-elliptic, $\left(2 \mathrm{I}_{1}^{*}, \mathrm{I}_{4}\right)$ elliptic and $\left(\mathrm{IV}^{*}, \mathrm{I}_{1}, \mathrm{I}_{3}\right)$ elliptic

In this case, we will show that $X$ has the dual graph of an Enriques surface of type VIII. If there is a quasi-elliptic fibration with singular fibers of type $\left(\mathrm{I}_{2}^{*}, 2 \mathrm{III}, 2 \mathrm{III}\right)$, we have the following configuration of (-2)-curves (use Table 6 and [ES04, Theorem 2.2]):

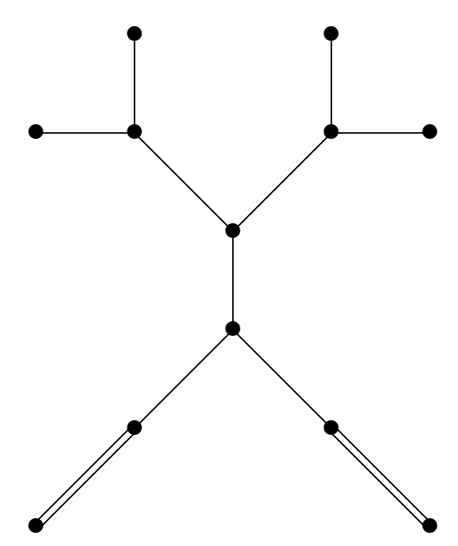

The special elliptic fibration induced by the diagram of type $\tilde{D}_{5}$ meeting the two curves at the bottom gives four more $(-2)$-curves. We leave it to the reader to check that the resulting intersection graph is of type VIII.

Next, we consider the case of a special elliptic fibration with singular fibers of type $\left(2 \mathrm{I}_{1}^{*}, \mathrm{I}_{4}\right)$. Let $C$ be the component of the fiber of type $2 \mathrm{I}_{1}^{*}$ with $C \cdot N=1$. We have seen in the proof of Lemma 3.12 that $A . N=1$; hence, $C$ is contained in $A$. The 2 -section $N$ has to meet a component $E$ of the fiber of type $\mathrm{I}_{4}$ twice since special genus 1 fibrations with a double fiber of type IV, $\mathrm{I}_{3}$ or $\mathrm{I}_{4}$ are not allowed by the above list. Therefore, there is a quasi-elliptic fibration with double singular fiber $2(N+E)$ of type III and curve of cusps $C$. This has to be a fibration with singular fibers of type $\left(\mathrm{I}_{2}^{*}, 2 \mathrm{III}, 2 \mathrm{III}\right)$ since the curve $C$ does not meet the component of the second fiber $F$ of type III which is also a component of the fiber of type $\mathrm{I}_{4}$ and $C$ cannot meet the other component of $F$ twice by Lemma 3.8(ii). Thus, we reduce this case to the previous one.

Starting with a quasi-elliptic fibration with singular fibers of type (I I $_{2}^{*}$ III, 2III) or (I I $_{2}^{*}$ III, III), we immediately get the existence of a special elliptic fibration with a singular double fiber of type $\mathrm{I}_{1}^{*}$ (use Table 6 and [ES04, Theorem 2.2]), returning us to the case above.

Finally, we consider the case of a special elliptic fibration with singular fibers of type (IV*, $\left.\mathrm{I}_{1}, \mathrm{I}_{3}\right)$. If the 2 -section meets two simple components of the fiber of type $\mathrm{IV}^{*}$, then there is a fibration with a double fiber of type $\mathrm{I}_{6}$, which gives a contradiction. Thus, the 2 -section meets either a simple component of the fiber of type $\mathrm{IV}^{*}$ twice or a double component once. In the first case, we get a quasi-elliptic fibration with a singular fiber of type 2III, and in the second case, we get a special elliptic fibration with a double fiber of type $I_{1}^{*}$. Both cases have already been dealt with. 


\section{T. Katsura, S. Kondō And G. Martin}

(13) Conductrix: $\stackrel{\bullet}{\bullet} \stackrel{1}{\bullet}$ Singularities: $D_{4}$

Possible special extremal fibrations: (I2, III, 2III) quasi-elliptic, (I I $_{2}^{*}$, III, III) quasi-elliptic and $\left(\mathrm{IV}^{*}, \mathrm{IV}\right)$ elliptic

This case does not occur on an Enriques surface with finite automorphism group. In fact, this follows immediately from the arguments in the last two paragraphs of the previous case.

(14) Conductrix: $\emptyset \quad$ Singularities: $D_{4}, 8 A_{1}$

Possible special extremal fibrations: (IV $\left.\mathrm{I}_{2}, \mathrm{I}_{6}\right)$ elliptic and (III, $\mathrm{I}_{8}$ ) elliptic

We will show that this case does not occur on an Enriques surface with finite automorphism group. We start from any of the two special fibrations and a special 2-section $N$. Consider the intersection of $N$ with the fibers of type $\mathrm{I}_{6}$ or $\mathrm{I}_{8}$. If $N$ meets two disjoint components of the fiber, then there is a genus 1 fibration with a double fiber of type $\mathrm{I}_{n}$, which gives a contradiction. Hence $N$ either meets a component of the fiber twice or meets two adjacent components. Then we can find a special genus 1 fibration with an additive double fiber of type III or IV. However, these fibrations are not allowed by the above list. Hence a surface with these singularities cannot have finite automorphism group.

(15) Conductrix: $\emptyset \quad$ Singularities: $12 A_{1}$

Possible special extremal fibrations: $\left(\mathrm{I}_{9}, \mathrm{I}_{1}, \mathrm{I}_{1}, \mathrm{I}_{1}\right)$ elliptic, $\left(\mathrm{I}_{5}, \mathrm{I}_{5}, \mathrm{I}_{1}, \mathrm{I}_{1}\right)$ elliptic, $\left(2 \mathrm{IV}, \mathrm{I}_{2}, \mathrm{I}_{6}\right)$ elliptic, (2III, $\left.\mathrm{I}_{8}\right)$ elliptic and $\left(\mathrm{I}_{3}, \mathrm{I}_{3}, \mathrm{I}_{3}, \mathrm{I}_{3}\right)$ elliptic

In this case, we will show that $X$ has the dual graph of an Enriques surface of type VII. If we start with a special fibration with singular fibers of type (2III, $\mathrm{I}_{8}$ ), the 2 -section has to meet two adjacent components of the fiber of type $\mathrm{I}_{8}$. Indeed, the twelve blow-ups for the dissolution all happen on the singular fibers, and the eight of them occurring on the fiber of type $\mathrm{I}_{8}$ are the blow-ups of the intersections of any two adjacent components. By Lemma 3.4(c), the $r$-invariant of any (-2)-curve is 2 . Thus we have to blow up two points on the special 2-section, and hence it has to meet such a point of intersection. The dual graph obtained is the one given in [Kon86, Figure 4.19.1(iii)]. From this configuration, we leave it to the reader to verify, using the above list, that the dual graph we obtain is the one of type VII (the argument is similar to [Kon86, (4.19.2)]).

Starting with a special extremal fibration with singular fibers of type $\left(2 \mathrm{IV}, \mathrm{I}_{2}, \mathrm{I}_{6}\right)$, we can check that there is a special fibration with double fiber of type 2III, which returns us to the case above. Indeed, if the 2-section meets distinct components of every fiber, we obtain either a genus 1 fibration with a double fiber of type $\mathrm{I}_{n}$, which is impossible, or a fibration with a singular fiber of type II*, which is not allowed by [ES04, Theorems 2.2 and 3.1].

For the other configurations, we also obtain a special elliptic fibration with a degenerate double fiber from the 2 -section and components of the fiber of type $\mathrm{I}_{n}$ with $n \geqslant 3$. Hence the argument of the previous two cases applies.

\section{Construction of vector fields}

In this section, we explain two methods to construct a candidate for a vector field $D$ on an algebraic surface $Y$ such that the quotient surface $Y^{D}$ becomes an Enriques surface. 


\section{Classification of EnRiques surfaces}

\subsection{Enriques surfaces with an elliptic pencil}

Let $f: Y \rightarrow \mathbf{P}^{1}$ be an elliptic surface with a section. Assume that $Y$ is either a $K 3$ surface or a rational surface. Then, the generic fiber is an elliptic curve $E$ over the field $k(t)$ with one variable $t$. Therefore, there exists a non-zero regular vector field $\delta$ on $E$ which we can regard as a non-zero rational vector field on $Y$. Taking a suitable vector field $g(t) \partial / \partial t$ and a suitable function $f(t)$ on $\mathbf{P}^{1}$, we look for a vector field

$$
D=f(t)\left\{g(t) \frac{\partial}{\partial t}+\delta\right\}
$$

such that $Y^{D}$ is birational to an Enriques surface. In many cases, double fibers of the Enriques surface $Y^{D}$ exist over the zero points of $g(t)$ by the theory of vector fields (cf. Proposition 2.1). In this way, we construct Enriques surfaces of type $\tilde{E}_{6}+\tilde{A}_{2}$ in Section 7 , of type VII in Section 8 and of type VIII in Section 9.

\subsection{Enriques surfaces with a quasi-elliptic pencil}

By Queen [Que71, Theorem 2], we have two normal forms for the generic fibers of a quasi-elliptic fiber space over the field $K=k(s)$ with a variable $s$ :

(1) $u^{2}=a+v+c v^{2}+d v^{4}$ with $a, c, d \in K$ and $d \notin k$,

(2) $u^{2}+u=a+d v^{4}$ with $a, d \in K$ and $d \notin k$.

Here, $u$ and $v$ are variables. Note that case (3) in Queen [Que71, Theorem 2] does not occur in our case because the transcendental degree of $K=k(s)$ over $k$ is 1 . For the relative generalized Jacobians of these quasi-elliptic surfaces, Queen [Que72, Theorem 1] showed the following:

- generalized Jacobian for (1): $u^{2}=v+c v^{2}+d v^{4}$

- generalized Jacobian for (2): $u^{2}+u=d v^{4}$.

Let us explain how to use case (1) to construct our Enriques surfaces (case (2) works similarly). By the change of coordinates $x=1 / v+c, y=u / v^{2}$, the generalized Jacobian for (1) is birational to

$$
y^{2}=x^{3}+c^{2} x+d,
$$

which is a Weierstrass normal form. By Bombieri-Mumford [BM76], the relative Jacobian of the quasi-elliptic Enriques surface is a rational surface. Therefore, this surface is birational to a rational quasi-elliptic surface in the list of Ito [Ito94, Proposition 5.1].

Starting from Ito's list of rational quasi-elliptic surfaces, we pursue the converse procedure to construct a candidate of an Enriques surface $X$, and using the candidate, we find a vector field $D$ on a rational surface $Y$ such that $Y^{D}$ is birational to the Enriques surface $X$. Using this technique, we will obtain Enriques surfaces of type $\tilde{E}_{8}$ in Section 10, of types $\tilde{E}_{7}+\tilde{A}_{1}^{(1)}$ and $\tilde{E}_{7}+\tilde{A}_{1}{ }^{(2)}$ in Section 11, of type $\tilde{D}_{8}$ in Section 12 and of type $\tilde{D}_{4}+\tilde{D}_{4}$ in Section 13. Of course, finding a suitable vector field $D$ is the hardest part of this construction and in the next subsection, we will give a detailed explanation on how to find a candidate for $D$ in the case of Enriques surfaces of type $\tilde{D}_{4}+\tilde{D}_{4}$.

\subsection{Example: Vector fields for Enriques surfaces of type $\tilde{D}_{4}+\tilde{D}_{4}$}

Following Ito [Ito94, Proposition 5.1], we take the rational quasi-elliptic surface defined by

$$
y^{2}=x^{3}+a^{4} s^{2} x+s^{3} \quad \text { with } a \in k .
$$




\section{T. Katsura, S. Kondō And G. Martin}

This quasi-elliptic surface has two singular fibers of type $\mathrm{I}_{0}^{*}$ (namely, of type $\tilde{D}_{4}$ ) over the points on $\mathbf{P}^{1}$ defined by $s=0$ and $s=\infty$. Taking the change of coordinates $x=1 / v+a^{2} s, y=s^{2} u / v^{2}$ and $s=1 / S$, we get

$$
u^{2}=S^{4} v+a^{2} S^{3} v^{2}+S v^{4} .
$$

Now, to introduce double fibers at $S=0$ and $S=\infty$ without changing the Jacobian fibration, we add a term $S^{7}+S^{3}$ and a parameter $b(b \neq 0)$ as follows:

$$
u^{2}=b^{2} S^{4} v+a^{2} S^{3} v^{2}+S v^{4}+S^{7}+S^{3} .
$$

We claim that these surfaces are quotients of rational surfaces by a vector field. For this purpose, we take the base change by the Frobenius morphism: $S=t^{2}$. Then, the surface becomes

$$
u^{2}+b^{2} t^{8} v+a^{2} t^{6} v^{2}+t^{2} v^{4}+t^{14}+t^{6}=0 .
$$

Therefore, by this equation, we have

$$
\left\{\left(u+a t^{3} v+t v^{2}+t^{7}+t^{3}\right) / b t^{4}\right\}^{2}=v .
$$

Now, by the change of coordinates $w=\left(u+a t^{3} v+t v^{2}+t^{7}+t^{3}\right) / b t^{4}, v=v, t=t$, we have

$$
u=b t^{4} w+a t^{3} w^{2}+t w^{4}+t^{7}+t^{3}, \quad v=w^{2} .
$$

Via these relations, $k(u, v, t)=k(w, t)$, which is a rational function field of two variables. Since

$$
u=b t^{4} w+a t^{3} w^{2}+t w^{4}+t^{7}+t^{3}, \quad S=t^{2}, \quad v=w^{2},
$$

we have

We put

$$
\frac{\partial u}{\partial w}=b t^{4}, \quad \frac{\partial u}{\partial t}=a t^{2} w^{2}+w^{4}+t^{6}+t^{2}
$$

$$
D^{\prime}=\left(1 / t^{3}\right)\left(b t^{4} \frac{\partial}{\partial t}+\left(a t^{2} w^{2}+w^{4}+t^{6}+t^{2}\right) \frac{\partial}{\partial w}\right) .
$$

Then, from equation (5.1), we see that $D^{\prime}(u)=0, D^{\prime}(v)=0, D^{\prime}(S)=0$ and $k(t, w)^{D^{\prime}}=$ $k(u, v, S)$. For later use, taking new coordinates $(x, y)$, we consider the change of coordinates $x=1 / t$ and $y=t / w$. Then, we have

$$
\frac{\partial}{\partial t}=x^{2} \frac{\partial}{\partial x}+x y \frac{\partial}{\partial y}, \quad \frac{\partial}{\partial w}=x y^{2} \frac{\partial}{\partial y} .
$$

By this change of coordinates, $D^{\prime}$ becomes

$$
D=\frac{1}{x^{2} y^{2}}\left(b x^{3} y^{2} \frac{\partial}{\partial x}+\left(a x^{2} y^{2}+x^{2}+x^{4} y^{4}+y^{4}+b x^{2} y^{3}\right) \frac{\partial}{\partial y}\right),
$$

where $a, b \in k, b \neq 0$. These are the vector fields that we will use in Section 13 to construct Enriques surfaces of type $\tilde{D}_{4}+\tilde{D}_{4}$.

\section{Equations of Enriques surfaces and their automorphisms}

In this section, we give a method to calculate the automorphism groups of Enriques surfaces $X$ with finite automorphism group in Theorems 1.3(b) and 1.4(b). We will use this method in cases of type $\tilde{E}_{6}+\tilde{A}_{2}$ (supersingular), type $\tilde{E}_{8}$ (supersingular and classical), type $\tilde{E}_{7}+\tilde{A}_{1}^{(1)}$ (supersingular), type $\tilde{D}_{8}$ (supersingular and classical) and type $\tilde{D}_{4}+\tilde{D}_{4}$.

Let $X$ be an Enriques surface, and assume that $X$ has a structure of a quasi-elliptic fibration $\varphi: X \rightarrow \mathbf{P}^{1}$. Let $t$ be a parameter of an affine line $\mathbf{A}^{1}$ in the base curve $\mathbf{P}^{1}$. We denote by $C$ the 


\section{Classification of EnRiques surfaces}

curve of cusps of the quasi-elliptic fibration and assume that over the point defined by $t=\infty$, it has a double fiber $2 F_{\infty}$. We assume that

$$
y^{2}=t x^{4}+g_{1}(t) x^{2}+g_{2}(t) x+g_{3}(t), \quad g_{1}(t), g_{2}(t), g_{3}(t) \in k[t]
$$

is the defining equation of an affine normal surface whose resolution of singularities is isomorphic to the open set $X \backslash\left(C \cup F_{\infty}\right)$ of $X$. Under these conditions, let $\sigma$ be an automorphism of X which preserves $F_{\infty}$. Then $\sigma$ preserves the structure of the quasi-elliptic fibration $\varphi: X \rightarrow \mathbf{P}^{1}$, and it acts on the base curve $\mathbf{P}^{1}$ with a fixed point at infinity:

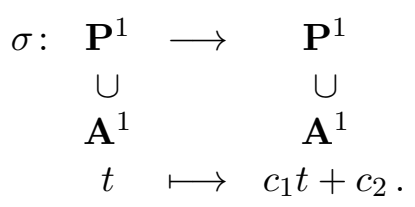

Here, $c_{1}$ and $c_{2}$ are elements of $k$ with $c_{1} \neq 0$.

We set $A=k[t, x, y] /\left(y^{2}+t x^{4}+g_{1}(t) x^{2}+g_{2}(t) x+g_{3}(t)\right)$. Then $A$ is normal by our assumption. As $k[t, x]$-module, we have

$$
A=k[t, x] \oplus k[t, x] y,
$$

which is a free $k[t, x]$-module. Since $\sigma$ preserve $C$ and $F_{\infty}$, it acts on the open set $X \backslash\left(C \cup F_{\infty}\right)$ of $X$.

Lemma 6.1. The automorphism $\sigma$ induces an automorphism of $\operatorname{Spec}(A)$.

Proof. We consider the change of coordinates $u=1 / x$ and $v=y / x^{2}$. Then, the equation becomes $v^{2}=t+g_{1}(t) u^{2}+g_{2}(t) u^{3}+g_{3}(t) u^{4}$, and the curve $C$ of cusps is given by $u=0$. The open set $X \backslash\left(C \cup F_{\infty}\right)$ is constructed as some blow-ups of $\operatorname{Spec}(A)$ :

$$
\pi: X \backslash\left(C \cup F_{\infty}\right) \longrightarrow \operatorname{Spec}(A) .
$$

Note that $\pi$ is surjective. Since $\sigma$ is an automorphism of $X \backslash\left(C \cup F_{\infty}\right)$, we have a morphism

$$
(\pi, \pi \circ \sigma): X \backslash\left(C \cup F_{\infty}\right) \longrightarrow \operatorname{Spec}(A) \times \operatorname{Spec}(A) .
$$

We denote the image of the morphism $(\pi, \pi \circ \sigma)$ by $\Gamma$. Let

$$
p_{i}: \operatorname{Spec}(A) \times \operatorname{Spec}(A) \longrightarrow \operatorname{Spec}(A), \quad i=1,2
$$

be the first and second projections. Then, restricting the projection $p_{1}$ to $\Gamma$, we have a morphism

$$
\left.p_{1}\right|_{\Gamma}: \Gamma \longrightarrow \operatorname{Spec}(A) \text {. }
$$

$\operatorname{Since} \operatorname{Spec}(A)$ is affine, the exceptional curves of the blow-ups collapse by the morphism $(\pi, \pi \circ \sigma)$. Therefore, the morphism $\left.p_{1}\right|_{\Gamma}$ is a finite birational morphism. Since $\operatorname{Spec}(A)$ is normal by our assumption, we see that by the Zariski main theorem, $\left.p_{1}\right|_{\Gamma}$ is an isomorphism. Therefore, we have a morphism $\left.\left.p_{2}\right|_{\Gamma} \circ p_{1}\right|_{\Gamma} ^{-1}: \operatorname{Spec}(A) \rightarrow \operatorname{Spec}(A)$ which is the automorphism induced by $\sigma$.

By this lemma, $\sigma$ acts on $\operatorname{Spec}(A)$ and induces an automorphism

$$
\sigma^{*}: A \longrightarrow A \text {. }
$$

Now we consider the generic fiber of $\varphi: X \rightarrow \mathbf{P}^{1}$. It is a curve of arithmetic genus 1 over $k(t)$ whose affine part is given by the equation (6.1). The curve $C$ of cusps gives a point $P_{\infty}$ of degree 2 on the curve of genus 1 . We denote by $\tilde{L}\left(P_{\infty}\right)$ the vector space over $k(t)$ associated with the linear system $\left|P_{\infty}\right|$ on the curve of genus 1 . By the Riemann-Roch theorem, we have $\operatorname{dim} \tilde{L}\left(P_{\infty}\right)=2$, 


\section{T. Katsura, S. Kondō And G. Martin}

and we see that 1 and $x$ give the basis of $\tilde{L}\left(P_{\infty}\right)$. Since $\sigma$ preserves the curve $C$ of cusps, $\sigma^{*}(x)$ is contained in $\tilde{L}\left(P_{\infty}\right)$. Therefore, there exist $d_{1}(t), d_{2}(t) \in k(t)$ such that

$$
\sigma^{*}(x)=d_{1}(t) x+d_{2}(t) .
$$

By (6.2) and (6.3), there exist $d_{3}(t, x), d_{4}(t, x) \in k[t, x]$ such that

$$
\sigma^{*}(x)=d_{3}(t, x)+d_{4}(t, x) y .
$$

Therefore, considering $\sigma^{*}(x)^{2}$, we have

$$
d_{1}(t)^{2} x^{2}+d_{2}(t)^{2}=d_{3}(t, x)^{2}+d_{4}(t, x)^{2}\left(t x^{4}+g_{1}(t) x^{2}+g_{2}(t) x+g_{3}(t)\right) .
$$

Since the right-hand-side is in $k[t, x]$, we see that $d_{1}(t)$ and $d_{2}(t)$ are also polynomials of $t$. Therefore, we see that $\sigma$ is of the following form:

$$
\sigma: \begin{cases}t \mapsto c_{1} t+c_{2}, & c_{1}, c_{2} \in k, \quad c_{1} \neq 0, \\ x \mapsto d_{1}(t) x+d_{2}(t), & d_{1}(t), d_{2}(t) \in k[t], \quad d_{1}(t) \not \equiv 0, \\ y \mapsto e_{1}(t, x) y+e_{2}(t, x), & e_{1}(t, x), e_{2}(t, x) \in k[t, x], \quad e_{1}(t, x) \not \equiv 0 .\end{cases}
$$

We will use this method in Theorems 7.9, 10.4, 10.9, 11.12, 12.5, 12.11and 13.4.

Remark 6.2. Let $X$ be an Enriques surface which has a structure of elliptic or quasi-elliptic fibration $\varphi: X \rightarrow \mathbf{P}^{1}$ defined by

$$
y^{2}+g_{0}(t) y=t x^{4}+g_{1}(t) x^{2}+g_{2}(t) x+g_{3}(t)
$$

with $g_{0}(t), g_{1}(t), g_{2}(t), g_{3}(t) \in k[t]$. Here, $t$ is a parameter of an affine line $\mathbf{A}^{1}$ in the base curve $\mathbf{P}^{1}$. We denote by $C$ the 2 -section defined by $x=\infty$ and by $F_{\infty}$ the fiber over the point on $\mathbf{P}^{1}$ defined by $t=\infty$. We assume that the equation is the defining equation of an affine normal surface whose resolution of singularities is isomorphic to the open set $X \backslash\left(C \cup F_{\infty}\right)$ of $X$. Under these conditions, let $\sigma$ be an automorphism of $\mathrm{X}$ which preserves the curve $C$ and the fiber $F_{\infty}$. Then, the automorphism $\sigma$ is also expressed in the form (6.4), and an argument similar to the above works.

We use the following trivial lemma.

Lemma 6.3. The ring $k[x, y]$ is a free $k\left[x^{2}, y^{2}\right]$-module of rank 4 . A basis is given by $1, x, y, x y$.

\section{Enriques surfaces of type $\tilde{E_{6}}+\tilde{A_{2}}$}

In Sections 7 through 13, we will construct the examples of Enriques surfaces given in Theorems 1.3(b) and 1.4(b). First, we consider the Enriques surfaces of type $\tilde{E}_{6}+\tilde{A}_{2}$, of type VII and of type VIII. In these cases, we use rational elliptic fibrations. Next, we consider the remaining cases. In these cases, we use a rational quasi-elliptic fibration. In this section, we construct Enriques surfaces of type $\tilde{E}_{6}+\tilde{A}_{2}$.

\subsection{Supersingular case}

We consider the relatively minimal non-singular complete elliptic surface $\psi: R \rightarrow \mathbf{P}^{1}$ associated with a Weierstrass equation

$$
y^{2}+s y=x^{3}
$$

with a parameter $s$. This surface is the unique rational elliptic surface with a singular fiber of type IV over the point given by $s=0$ and a singular fiber of type IV $^{*}$ over the point given 


\section{Classification of EnRiques surfaces}

by $s=\infty$ (Lang [Lan94, Section 2]). Note that all non-singular fibers are supersingular elliptic curves. We consider the base change of $\psi: R \rightarrow \mathbf{P}^{1}$ by $s=t^{2}$. Then, we have the elliptic surface defined by

$$
y^{2}+t^{2} y=x^{3}
$$

We consider the relatively minimal non-singular complete model of this elliptic surface:

$$
f: \tilde{R} \longrightarrow \mathbf{P}^{1} \text {. }
$$

By considering the change of coordinates defined by $x^{\prime}=x / t^{2}, y^{\prime}=y / t^{3}, t^{\prime}=1 / t$, we have

$$
y^{\prime 2}+t^{\prime} y^{\prime}=x^{\prime 3} .
$$

Thus the surface $\tilde{R}$ is isomorphic to $R$. The rational elliptic surface $f: \tilde{R} \rightarrow \mathbf{P}^{1}$ has a singular fiber of type $\mathrm{IV}^{*}$ over the point given by $t=0$ and a singular fiber of type IV over the point given by $t=\infty$.

The elliptic surface $f: \tilde{R} \rightarrow \mathbf{P}^{1}$ has three sections $s_{i}(i=0,1,2)$ given as follows:

$$
s_{0} \text { : the zero section, } s_{1}: x=y=0, s_{2}: x=0, y=t^{2} .
$$

On the singular elliptic surface (7.1), we denote by $F_{0}$ the fiber over the point defined by $t=0$ and by $F_{\infty}$ the fiber over the point defined by $t=\infty$. Both $F_{0}$ and $F_{\infty}$ are irreducible, and on each $F_{i}(i=0, \infty)$, the surface (7.1) has only one singular point $P_{i}$. The surface $\tilde{R}$ is the surface obtained by the minimal resolution of singularities of the surface (7.1). We denote the proper transform of $F_{i}$ on $\tilde{R}$ again by $F_{i}$ if no confusion can occur. We have six exceptional curves $E_{0, k}(k=1,2, \ldots, 6)$ over the point $P_{0}$ such that $F_{0}$ and these six exceptional curves form a singular fiber of type $\mathrm{IV}^{*}$ of the elliptic surface $f: \tilde{R} \rightarrow \mathbf{P}^{1}$ as follows: The blow-up at the singular point $P_{0}$ gives one exceptional curve $E_{0,1}$, and the surface is non-singular along $F_{0}$ and has a unique singular point $P_{0,1}$ on $E_{0,1}$. The blow-up at the singular point $P_{0,1}$ gives two exceptional curves $E_{0,2}$ and $E_{0,3}$. We denote the proper transform of $E_{0,1}$ by $E_{0,1}$. The three curves $E_{0,1}, E_{0,2}$ and $E_{0,3}$ meet at one point $P_{0,2}$ which is a singular point of the resulting surface. The blow-up at the singular point $P_{0,2}$ again gives two exceptional curves $E_{0,4}$ and $E_{0,5}$. The three curves $E_{0,1}, E_{0,4}$ and $E_{0,5}$ meet at one point $P_{0,3}$ which is a singular point of the resulting surface. The curve $E_{0,2}$ (respectively, $E_{0,3}$ ) intersects $E_{0,4}$ (respectively, $E_{0,5}$ ) and does not meet other curves. Finally, the blow-up at the singular point $P_{0,3}$ gives an exceptional curve $E_{0,6}$, and the resulting surface is non-singular over these curves. The curve $E_{0,6}$ meets $E_{0,1}, E_{0,4}$ and $E_{0,5}$ transversally. The dual graph of the curves $F_{0}, E_{0,1}, \ldots, E_{0,6}$ is of type $\tilde{E}_{6}$. Thus, the cycle

$$
F_{0}+E_{0,2}+E_{0,3}+2\left(E_{0,1}+E_{0,4}+E_{0,5}\right)+3 E_{0,6}
$$

is a singular fiber of type $\mathrm{IV}^{*}$. On the other hand, the blow-up at the singular point $P_{\infty}$ gives two exceptional curves $E_{\infty, 1}$ and $E_{\infty, 2}$. The resulting surface is now relatively minimal and nonsingular, that is, nothing but $\tilde{R}$. The three curves $F_{\infty}, E_{\infty, 1}$ and $E_{\infty, 2}$ form a singular fiber of type IV. The configuration of these curves is as in Figure 1.

The sections $s_{i}$ have self-intersection number -1 , and the fiber components have self-intersection number -2 .

Now, we consider a rational vector field on $\tilde{R}$ induced from

$$
D=\frac{\partial}{\partial t}+t^{2} \frac{\partial}{\partial x}
$$

Then, we have $D^{2}=0$; that is, $D$ is 2-closed. However, $D$ has an isolated singularity at the 


\section{T. Katsura, S. Kondō And G. Martin}

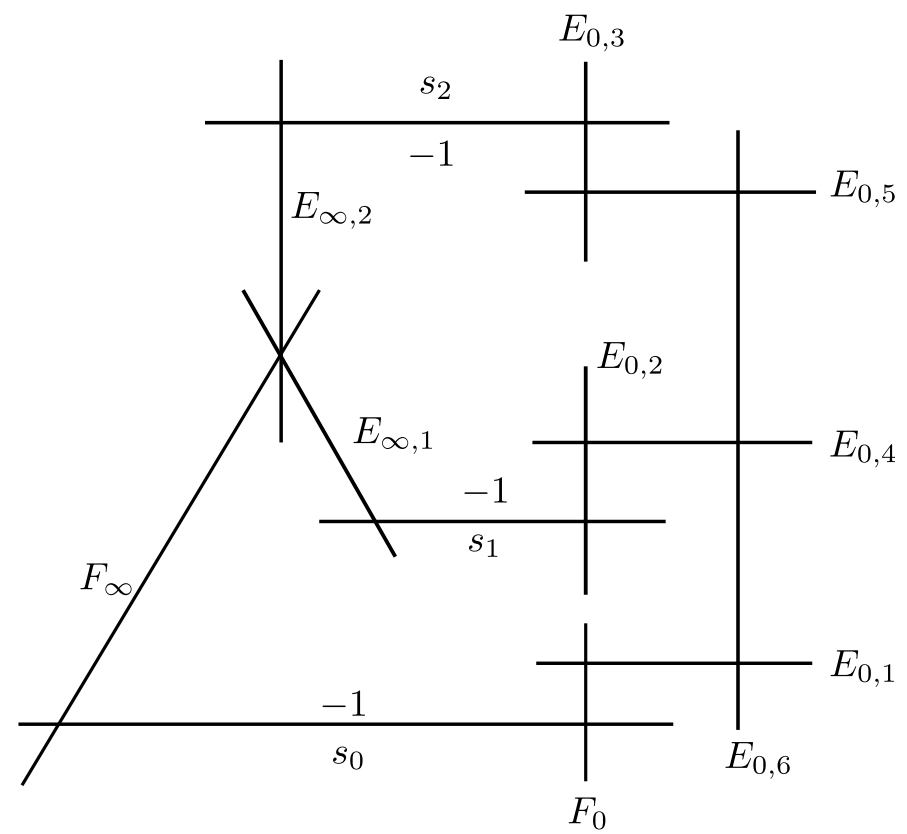

Figure 1

point $P$ which is the singular point of the fiber of type IV, that is, the intersection point of three curves $F_{\infty}, E_{\infty, 1}$ and $E_{\infty, 2}$ (note that $(t, x)$ is not a local parameter along the fiber defined by $t=0$ ). To resolve this singularity, we first blow up at $P$. Denote by $E_{\infty, 3}$ the exceptional curve. We denote the proper transforms of $F_{\infty}, E_{\infty, 1}$ and $E_{\infty, 2}$ by the same symbols. Then blow up at three points $E_{\infty, 3} \cap\left(F_{\infty}+E_{\infty, 1}+E_{\infty, 2}\right)$. Let $Y$ be the resulting surface and $\psi: Y \rightarrow \tilde{R}$ the successive blow-ups. We denote by $E_{\infty, 4}, E_{\infty, 5}$ and $E_{\infty, 6}$ the exceptional curves over the points $E_{\infty, 3} \cap F_{\infty}, E_{\infty, 3} \cap E_{\infty, 1}$ and $E_{\infty, 3} \cap E_{\infty, 2}$, respectively. Then we have the configuration in Figure 2. In that figure, we give the self-intersection numbers of the curves except for the curves with self-intersection number -2 , and the thick lines are integral curves with respect to $D$.

Now, according to the above blow-ups, we see the following.

Lemma 7.1. (i) The divisorial part $(D)$ on $Y$ is given by

$$
-2\left(E_{0,1}+E_{0,4}+E_{0,5}+E_{0,6}+E_{\infty, 3}+E_{\infty, 4}+E_{\infty, 5}+E_{\infty, 6}\right)-\left(F_{\infty}+E_{\infty, 1}+E_{\infty, 2}\right) .
$$

(ii) The integral curves in Figure 2 are $E_{0,1}, E_{0,4}, E_{0,5}, F_{\infty}, E_{\infty, 1}, E_{\infty, 2}, E_{\infty, 3}$.

LEMMA 7.2. (i) $(D)^{2}=-12$.

(ii) The canonical divisor $K_{Y}$ of $Y$ is given by

$$
K_{Y}=-2\left(E_{\infty, 3}+E_{\infty, 4}+E_{\infty, 5}+E_{\infty, 6}\right)-\left(F_{\infty}+E_{\infty, 1}+E_{\infty, 2}\right) .
$$

(iii) $K_{Y} \cdot(D)=-4$.

Lemma 7.3. The vector field $D$ is divisorial, and the quotient surface $Y^{D}$ is non-singular.

Proof. Since $\tilde{R}$ is a rational elliptic surface and $Y$ is the blow-up at four points, we have $c_{2}(Y)=$ 16. Using $(D)^{2}=-12, K_{Y} \cdot(D)=-4$ and the equation (2.3), we have

$$
16=c_{2}(Y)=\operatorname{deg}\langle D\rangle-K_{Y} \cdot(D)-(D)^{2}=\operatorname{deg}\langle D\rangle+4+12 .
$$

Therefore, we have $\operatorname{deg}\langle D\rangle=0$. This means that $D$ is divisorial and that $Y^{D}$ is non-singular. 


\section{Classification of Enriques surfaces}

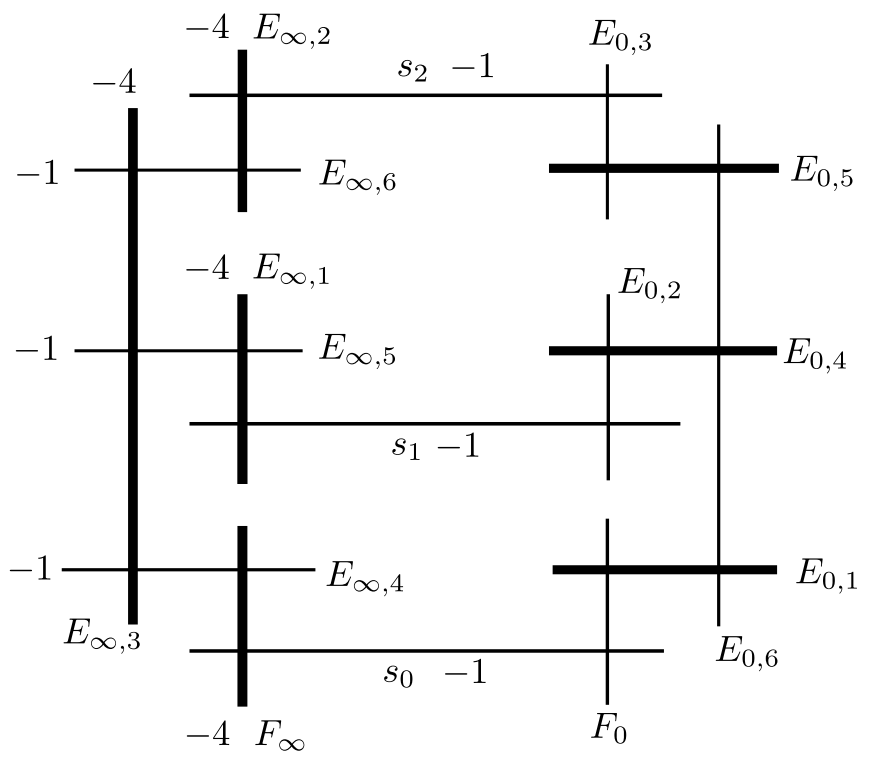

Figure 2

Let $\pi: Y \rightarrow Y^{D}$ be the natural map. By the result on the canonical divisor formula (2.2), we have

$$
K_{Y}=\pi^{*} K_{Y^{D}}+(D) \text {. }
$$

Lemma 7.4. (i) The images of the curves $E_{0,1}, E_{0,4}, E_{0,5}$ in $Y^{D}$ are exceptional curves.

(ii) The self-intersection numbers of the images of $F_{0}, E_{0,2}, E_{0,3}, E_{0,6}$ in $Y^{D}$ are -4 .

(iii) The self-intersection numbers of the images of $F_{\infty}, E_{\infty, i}(i=1, \ldots, 6)$ and the three sections $s_{i}(i=0,1,2)$ in $Y^{D}$ are -2 .

Proof. The assertions follow from Proposition 2.1 and Lemma 7.1(ii).

Let $E_{0,1}^{\prime}, E_{0,4}^{\prime}, E_{0,5}^{\prime}, E_{0,6}^{\prime}$ be the images of $E_{0,1}, E_{0,4}, E_{0,5}, E_{0,6}$ in $Y^{D}$, respectively. Then we have the configuration in Figure 3, in which we give the self-intersection numbers of the curves except for the curves with self-intersection number -2 .

Let $\varphi_{1}: Y^{D} \rightarrow X^{\prime}$ be the blow-down of $E_{0,1}^{\prime}, E_{0,4}^{\prime}, E_{0,5}^{\prime}$. Then the image of $E_{0,6}^{\prime}$ in $X^{\prime}$ is an exceptional curve. Let $\varphi_{2}: X^{\prime} \rightarrow X$ be the blow-down of this exceptional curve. Now we have the following diagram:

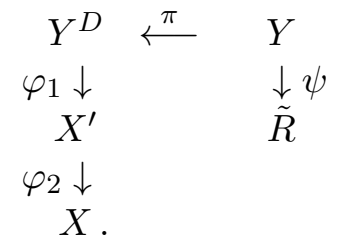

We have thirteen $(-2)$-curves $E_{1}, \ldots, E_{13}$ with the self-intersection number -2 which form Figure 4.

Then, we have

$$
\begin{aligned}
K_{Y^{D}} & =\varphi_{1}^{*}\left(K_{X^{\prime}}\right)+E_{0,1}+E_{0,4}+E_{0,5} \\
& =\varphi_{1}^{*} \circ \varphi_{2}^{*}\left(K_{X}\right)+E_{0,6}+2\left(E_{0,1}+E_{0,4}+E_{0,5}\right) .
\end{aligned}
$$


T. Katsura, S. Kondō And G. Martin

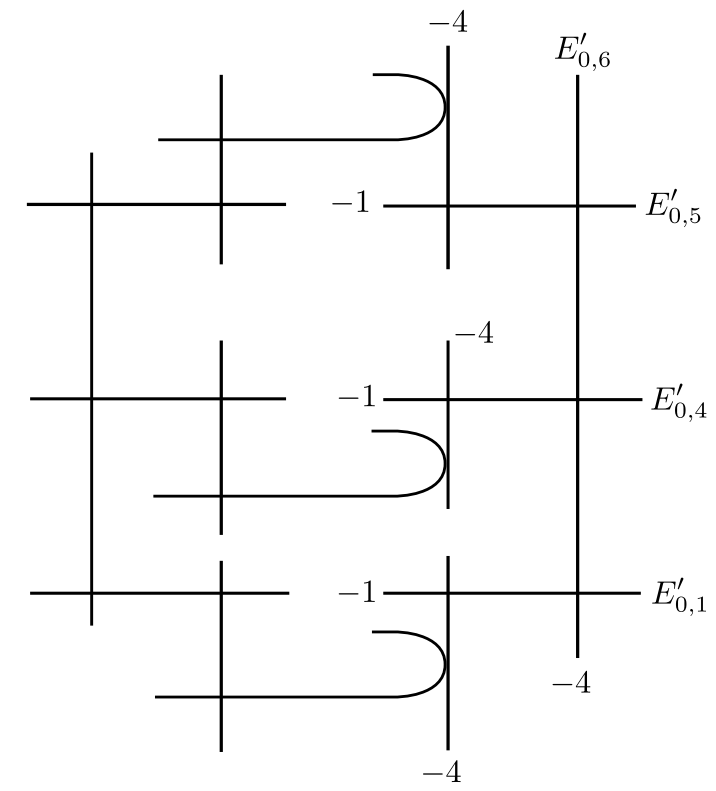

Figure 3

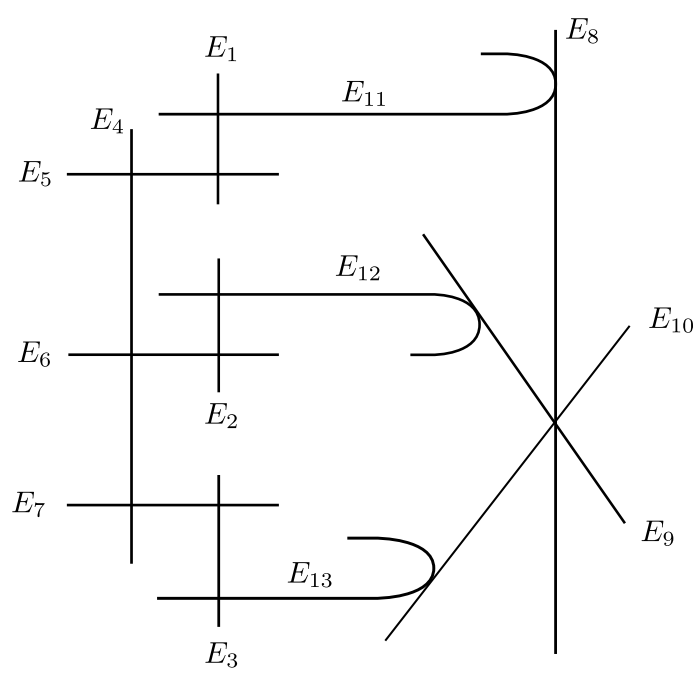

Figure 4

Lemma 7.5. The canonical divisor $K_{X}$ of $X$ is numerically equivalent to zero.

Proof. By Lemma 7.2(ii), we have

$$
K_{Y}=-2\left(E_{\infty, 3}+E_{\infty, 4}+E_{\infty, 5}+E_{\infty, 6}\right)-\left(F_{\infty}+E_{\infty, 1}+E_{\infty, 2}\right) .
$$

On the other hand, we have

$$
\begin{aligned}
K_{Y} & =\pi^{*} K_{Y^{D}}+(D)=\pi^{*}\left(\varphi_{1}^{*} \circ \varphi_{2}^{*}\left(K_{X}\right)+E_{0,6}+2\left(E_{0,1}+E_{0,4}+E_{0,5}\right)\right)+(D) \\
& =\pi^{*}\left(\varphi_{1}^{*} \circ \varphi_{2}^{*}\left(K_{X}\right)\right)+2\left(E_{0,6}+E_{0,1}+E_{0,4}+E_{0,5}\right)+(D)=\pi^{*}\left(\varphi_{1}^{*} \circ \varphi_{2}^{*}\left(K_{X}\right)\right)+K_{Y} .
\end{aligned}
$$

Here, we use the fact that $E_{0,1}, E_{0,4}, E_{0,5}$ are integral and $E_{0,6}$ is non-integral (Lemma 7.1(ii) and Lemma 2.1). Therefore, $K_{X}$ is numerically equivalent to zero. 


\section{Classification of EnRiques surfaces}

Lemma 7.6. The surface $X$ has $b_{2}(X)=10$.

Proof. Since $\pi: Y \rightarrow Y^{D}$ is finite and purely inseparable, the étale cohomology of $Y$ is isomorphic to the étale cohomology of $Y^{D}$. Therefore, we have $b_{1}\left(Y^{D}\right)=b_{1}(Y)=0, b_{3}\left(Y^{D}\right)=b_{3}(Y)=0$ and $b_{2}\left(Y^{D}\right)=b_{2}(Y)=14$. Since $\varphi_{2} \circ \varphi_{1}$ is the blow-down of four exceptional curves, we see that $b_{0}(X)=b_{4}(X)=1, b_{1}(X)=b_{3}(X)=0$ and $b_{2}(X)=10$.

TheOREM 7.7. With the notation above, $X$ is a supersingular Enriques surface.

Proof. Since $K_{X}$ is numerically trivial, $X$ is minimal and the Kodaira dimension $\kappa(X)$ is equal to 0 . Since $b_{2}(X)=10$, we have that $X$ is an Enriques surface. Since $Y$ is a rational surface, $X$ is either supersingular or classical. Consider the elliptic fibration $g: X \rightarrow \mathbf{P}^{1}$ induced by $f: \tilde{R} \rightarrow \mathbf{P}^{1}$. Note that the fiber over the point given by $t=\infty$ is a double fiber of type $\mathrm{IV}^{*}$ and that the fiber over the point given by $t=0$ is a simple fiber of type IV. Since $f$ has only two singular fibers and any smooth fiber is a supersingular elliptic curve, the other fibers of $g$ are smooth and supersingular elliptic curves by Lemma 2.6, and hence they are simple by Proposition 2.5. Therefore, $X$ is a supersingular Enriques surface by Proposition 2.5.

The dual graph of the thirteen $(-2)$-curves $E_{1}, \ldots, E_{13}$ is as in Figure 5 . We can easily

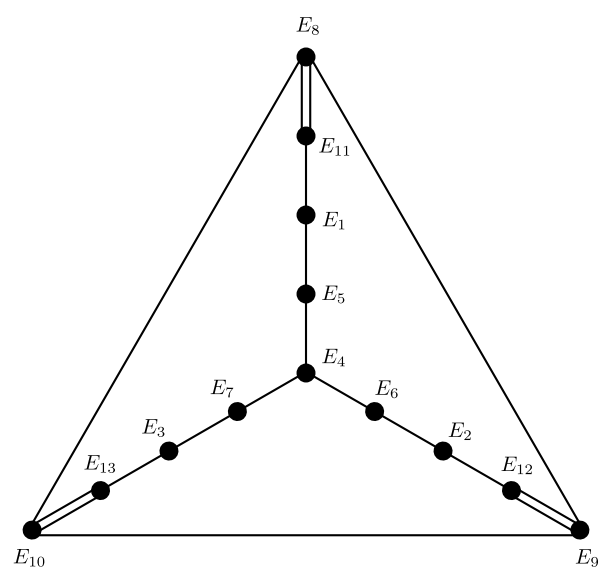

Figure 5

determine all divisors of Kodaira type in Figure 5. It follows from Proposition 2.4 that each of them defines a genus 1 fibration on $X$. Thus, on $X$, there exist exactly one elliptic fibration with singular fibers of type $\left(2 \mathrm{IV}^{*}, \mathrm{IV}\right)$, defined by the linear system $\left|E_{8}+E_{9}+E_{10}\right|$, and three quasielliptic fibrations with singular fibers of type (III*, 2III), defined by $\left|2\left(E_{9}+E_{12}\right)\right|,\left|2\left(E_{8}+E_{11}\right)\right|$ and $\left|2\left(E_{10}+E_{13}\right)\right|$.

We now have the following theorem.

ThEOREM 7.8. The automorphism group $\operatorname{Aut}(X)$ is finite, and $X$ contains exactly thirteen (-2)-curves.

Proof. Consider the dual graph $\Gamma$ of the thirteen (-2)-curves in Figure 5. By Remark 2.15, the graph $\Gamma$ is non-degenerate. We can easily prove that any maximal parabolic subdiagram in $\Gamma$ is of type $\tilde{E}_{6} \oplus \tilde{A}_{2}$ or of type $\tilde{E}_{7} \oplus \tilde{A}_{1}$. It follows from Propositions $2.2,2.14$ and 2.16 that $\operatorname{Aut}(X)$ is finite and that $X$ contains exactly thirteen $(-2)$-curves.

TheOREM 7.9. The automorphism group $\operatorname{Aut}(X)$ is isomorphic to $\mathbf{Z} / 5 \mathbf{Z} \times \mathfrak{S}_{3}$, and the numerically trivial automorphism group $\operatorname{Aut}_{n t}(X)$ is isomorphic to $\mathbf{Z} / 5 \mathbf{Z}$. 


\section{T. Katsura, S. Kondō And G. Martin}

Proof. To calculate $\operatorname{Aut}(X)$, we first give an equation of a birational model $X$ which has the structure of a special elliptic fibration with singular fibers of type $\left(2 \mathrm{IV}^{*}, \mathrm{IV}\right)$. Then we calculate the group of automorphisms preserving this fibration and a 2-section. Finally, using this result, we determine the automorphism group of $X$.

We consider the elliptic surface defined by the equation (7.1) and the vector field given in (7.3). Put $T=t^{2}, u=x+t^{3}, v=y+t x^{2}$. Then, we have $D(T)=0, D(u)=0, D(v)=0$, and we have the relation

$$
v^{2}+T v=T u^{4}+u^{3}+T^{3} u+T^{7} .
$$

Since we have $k(x, y, t)^{D}=k(u, v, T)$, the quotient surface by $D$ is birational to the surface defined by the equation (7.4). Note that the minimal normal completion of this surface is a normal elliptic surface $f: X \rightarrow \mathbf{P}^{1}$ which is birational to our Enriques surface. We replace variables $u, v, T$ by new variables $x, y, t$, respectively, for convenience, and set

$$
A=k[t, x, y] /\left(y^{2}+t y+t x^{4}+x^{3}+t^{3} x+t^{7}\right) .
$$

Let $\sigma$ be an automorphism of our Enriques surface. The double fiber of $f$ of type $\mathrm{IV}^{*}$, denoted by $2 F_{\infty}$, exists over the point defined by $t=\infty$. Since $\sigma$ preserves the diagram of $(-2)$-curves, $\sigma$ preserves $2 F_{\infty}$. Therefore, $\sigma$ preserves the structure of this elliptic surface. We denote by $\tilde{C}$ the 2 -section at infinity and assume that $\sigma$ preserves $\tilde{C}$. Then, as in the case of a quasi-elliptic surface, $\sigma$ has the form in (6.4) (cf. Remark 6.2). Moreover, since this elliptic surface has a unique second singular fiber over the point defined by $t=0$, the automorphism $\sigma$ also preserves the singular fiber. Hence we know $c_{2}=0$, and we have $\sigma^{*}(t)=c_{1} t$.

Therefore, together with the equation (7.4), we have an identity

$$
\begin{aligned}
& e_{1}(t, x)^{2}\left(t y+t x^{4}+x^{3}+t^{3} x+t^{7}\right)+e_{2}(t, x)^{2}+c_{1} t\left(e_{1}(t, x) y+e_{2}(t, x)\right) \\
& \quad=c_{1} t\left(d_{1}(t) x+d_{2}(t)\right)^{4}+\left(d_{1}(t) x+d_{2}(t)\right)^{3}+\left(c_{1} t\right)^{3}\left(d_{1}(t) x+d_{2}(t)\right)+\left(c_{1} t\right)^{7} .
\end{aligned}
$$

The ring $A$ is a free $k[t, x]$-module, and 1 and $y$ are linearly independent over $k[t, x]$. Taking the coefficient of $y$, we have $e_{1}(t, x)^{2} t+c_{1} t e_{1}(t, x)=0$. Since $e_{1}(t, x) \not \equiv 0$, we have $e_{1}(t, x)=c_{1} \in k^{*}$. Therefore, we have

$$
\begin{aligned}
& c_{1}^{2}\left(t x^{4}+x^{3}+t^{3} x+t^{7}\right)+e_{2}(t, x)^{2}+c_{1} t e_{2}(t, x) \\
& \quad=c_{1} t\left(d_{1}(t) x+d_{2}(t)\right)^{4}+\left(d_{1}(t) x+d_{2}(t)\right)^{3}+\left(c_{1} t\right)^{3}\left(d_{1}(t) x+d_{2}(t)\right)+\left(c_{1} t\right)^{7} .
\end{aligned}
$$

As a polynomial of $x$, if $e_{2}(t, x)$ has a term of degree greater than or equal to 3 , then $e_{2}(t, x)^{2}$ has a term of degree greater than or equal to 6 . We cannot kill this term in the equation. Therefore, we can put $e_{2}(t, x)=a_{0}(t)+a_{1}(t) x+a_{2}(t) x^{2}$ with $a_{0}(t), a_{1}(t), a_{2}(t) \in k[t]$. We take terms which contain only the variable $t$. Then, we have an equality

$$
c_{1}^{2} t^{7}+a_{0}(t)^{2}+c_{1} t a_{0}(t)=c_{1} t d_{2}(t)^{4}+d_{2}(t)^{3}+c_{1}^{3} t^{3} d_{2}(t)+c_{1}^{7} t^{7} .
$$

Put $\operatorname{deg} d_{2}(t)=\ell$. Suppose $\ell \geqslant 2$. Then, the right-hand side has an odd term whose degree is equal to $4 \ell+1 \geqslant 9$. Therefore, the left-hand side must have an odd term which is of degree $4 \ell+1$. This means $\operatorname{deg} a_{0}(t)=4 \ell$. However, in the equation we cannot kill the term of degree $8 \ell$ which comes from $a_{0}(t)^{2}$. Therefore, we can put $d_{2}(t)=b_{0}+b_{1} t$ with $b_{0}, b_{1} \in k$. Then, the equation becomes

$$
a_{0}(t)^{2}+c_{1} t a_{0}(t)+c_{1}^{2} t^{7}=c_{1} b_{0}^{4} t+c_{1} b_{1}^{4} t^{5}+b_{0}^{3}+b_{0}^{2} b_{1} t+b_{0} b_{1}^{2} t^{2}+b_{1}^{3} t^{3}+c_{1}^{3} b_{0} t^{3}+c_{1}^{3} b_{1} t^{4}+c_{1}^{7} t^{7} .
$$

If $\operatorname{deg} a_{0}(t) \geqslant 4$, we cannot kill the term of degree greater than or equal to 8 which comes 


\section{Classification of EnRiques surfaces}

from $a_{0}(t)^{2}$. Therefore, we can put $a_{0}(t)=\alpha_{0}+\alpha_{1} t+\alpha_{2} t^{2}+\alpha_{3} t^{3}$. Then, we have equations

$$
\begin{gathered}
c_{1}^{2}=c_{1}^{7}, \quad \alpha_{3}^{2}=0, \quad 0=c_{1} b_{1}^{4}, \quad \alpha_{2}^{2}+c_{1} \alpha_{3}=c_{1}^{3} b_{1}, \quad c_{1} \alpha_{2}=b_{1}^{3}+c_{1}^{3} b_{0}, \\
\alpha_{1}^{2}+c_{1} \alpha_{1}=b_{0} b_{1}^{2}, \quad c_{1} \alpha_{0}=c_{1} b_{0}^{4}+b_{0}^{2} b_{1}, \quad \alpha_{0}^{2}=b_{0}^{3} .
\end{gathered}
$$

Solving these equations, we have

$$
b_{0}=0, \quad b_{1}=0, \quad \alpha_{0}=0, \quad \alpha_{2}=0, \quad \alpha_{3}=0, \quad c_{1}^{5}=1, \quad \alpha_{1}=0 \text { or } c_{1} .
$$

Therefore, we have $c_{1}=\zeta, e_{1}(t, x)=\zeta, a_{0}(t)=0$ or $\zeta t, d_{2}(t)=0$ with $\zeta^{5}=1, \zeta \in k$. Substituting these into the original equation, we have

$$
\begin{aligned}
& \zeta^{2}\left(t x^{4}+x^{3}+t^{3} x\right)+a_{1}(t)^{2} x^{2}+a_{2}(t)^{2} x^{4}+\zeta t a_{1}(t) x+\zeta t a_{2}(t) x^{2} \\
& \quad=\zeta t d_{1}(t)^{4} x^{4}+d_{1}(t)^{3} x^{3}+\zeta^{3} t^{3} d_{1}(t) x .
\end{aligned}
$$

Considering the coefficients of $x^{4}$, we have $\zeta^{2} t+a_{2}(t)^{2}+\zeta t d_{1}(t)^{4}=0$. Therefore, we have $a_{2}(t)=0$ and $d_{1}(t)=\zeta^{4}$. Considering the coefficients of $x^{2}$, we have $a_{1}(t)=0$. Therefore, we have

$$
c_{1}=\zeta, \quad d_{1}(t)=\zeta^{4}, \quad d_{2}(t)=0, \quad e_{1}(t, x)=\zeta, \quad e_{2}(t, x)=0 \text { or } \zeta t .
$$

Now, we set

$$
\begin{gathered}
\sigma: t \mapsto \zeta t, \quad x \mapsto \zeta^{4} x, \quad y \mapsto \zeta y, \\
\tau: t \mapsto t, \quad x \mapsto x, \quad y \mapsto y+t .
\end{gathered}
$$

Then, we have

$$
\sigma \circ \tau: t \mapsto \zeta t, \quad x \mapsto \zeta^{4} x, \quad y \mapsto \zeta y+\zeta t
$$

and $\langle\sigma \circ \tau\rangle \cong \mathbf{Z} / 10 \mathbf{Z}$. We now conclude that the group of automorphisms of $X$ preserving a 2-section of the elliptic fibration with singular fibers of type (VI*, VI) is isomorphic to $\mathbf{Z} / 10 \mathbf{Z}$.

It is easy to see that the automorphism $\tau$ of order 2 is not numerically trivial. Indeed, if it were, it would preserve all 2 -sections of $f$. Therefore, it would fix at least two points on them, namely the intersection with the reducible fibers (note that they touch the fiber of type IV since the other fibrations on this surface have a fiber of type III). Hence $\tau$ would fix all 2-sections of $f$ pointwise, and therefore it would also fix a general fiber of $f$ by Lemma 2.13. This gives a contradiction; hence, $\tau$ is not numerically trivial.

Finally, consider the relative Jacobian variety of $f: X \rightarrow \mathbf{P}^{1}$. It has singular fibers of types IV and $\mathrm{IV}^{*}$, and the Mordell-Weil group is isomorphic to $\mathbf{Z} / 3 \mathbf{Z}$ (cf. Ito [Ito02]). We denote by $\rho$ a generator of the group. It acts on $X$ and permutes the three smooth rational 2 -sections because if it fixes a 2 -section, then it is contained in the above group $\mathbf{Z} / 10 \mathbf{Z}$.

Therefore, by considering the action of the subgroup $\langle\tau, \rho\rangle$ generated by $\tau$ and $\rho$ on the dual graph of $(-2)$-curves, we see that $\langle\tau, \rho\rangle$ acts as the symmetric group $\mathfrak{S}_{3}$ of degree 3 . The automorphism $\sigma$ is numerically trivial because the dual graph of nodal curves has no symmetries of order 5 . We conclude that $\operatorname{Aut}(X)$ is an extension of $\mathfrak{S}_{3}$ by $\mathbf{Z} / 5 \mathbf{Z}$. By the above calculations, $\tau$ commutes with $\sigma$, hence $\operatorname{Aut}(X) \cong \mathbf{Z} / 5 \mathbf{Z} \times \mathfrak{S}_{3}$.

Remark 7.10. Note that $\operatorname{Aut}_{\mathrm{ct}}(X)=\operatorname{Aut}_{\mathrm{nt}}(X)$ because $X$ is supersingular. The numerically trivial automorphism $\sigma$ of order 5 found here leads to one of the exceptions in [DM19]. 


\section{T. Katsura, S. Kondō And G. Martin}

\subsection{Classical case}

We consider the relatively minimal non-singular complete elliptic surface $\psi: R \rightarrow \mathbf{P}^{1}$ associated with the Weierstrass equation

$$
y^{2}+x y+s y=x^{3}
$$

with a parameter $s$. This surface is a rational elliptic surface with a singular fiber of type $\mathrm{I}_{3}$ over the point given by $s=0$, a singular fiber of type $\mathrm{I}_{1}$ over the point given by $s=1$ and a singular fibers of type $\mathrm{IV}^{*}$ over the point given by $s=\infty$ (cf. Lang [Lan94, Section 2]). We consider the base change of $\psi: R \rightarrow \mathbf{P}^{1}$ by $s=t^{2}$. Then, we have the elliptic surface associated with the Weierstrass equation

$$
y^{2}+x y+t^{2} y=x^{3} .
$$

We consider the relatively minimal non-singular complete model of this elliptic surface:

$$
f: \tilde{R} \longrightarrow \mathbf{P}^{1} \text {. }
$$

The rational elliptic surface $f: \tilde{R} \rightarrow \mathbf{P}^{1}$ has a singular fiber of type $\mathrm{I}_{6}$ over the point given by $t=0$, a singular fiber of type $\mathrm{I}_{2}$ over the point given by $t=1$ and a singular fiber of type IV over the point given by $t=\infty$ (see Figure 6). The fibration $f$ has six sections. In Figure 6 , $(-1)$-curves denote the 0 -section and the two sections defined by the equations

$$
x=y=0, \quad x=y+t^{2}=0 .
$$

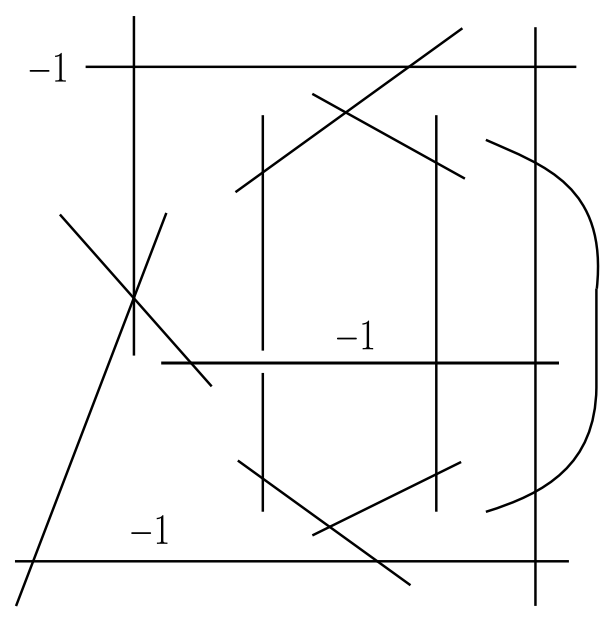

Figure 6

Now, we consider a rational vector field on $\tilde{R}$ defined by

$$
D=D_{a}=(t+a) \frac{\partial}{\partial t}+\left(x+t^{2}\right) \frac{\partial}{\partial x},
$$

where $a \in k \backslash\{0,1\}$. We see that $D^{2}=D$; that is, $D$ is 2-closed. Note that the non-singular fiber $F_{a}$ over the point defined by $t=a$ is integral with respect to $D$. The vector field $D$ has an isolated singularity at the point $P$ which is the singular point of the fiber of type IV. Denote by $F_{\infty}$, $E_{\infty, 1}$ and $E_{\infty, 2}$ the three components of the singular fiber of type IV. Then $P$ is the intersection point of these three curves. To resolve this singularity, we first blow up at $P$. Denote by $E_{\infty, 3}$ the exceptional curve. We denote the proper transforms of $F_{\infty}, E_{\infty, 1}$ and $E_{\infty, 2}$ by the same symbols. Then blow up at the three points $E_{\infty, 3} \cap\left(F_{\infty}+E_{\infty, 1}+E_{\infty, 2}\right)$. Let $Y$ be the resulting surface and $\psi: Y \rightarrow \tilde{R}$ the successive blow-ups. We denote by the same symbol $D$ the induced 


\section{Classification of EnRiques surfaces}

vector field on $Y$. We denote by $E_{\infty, 4}, E_{\infty, 5}$ and $E_{\infty, 6}$ the exceptional curves over the points $E_{\infty, 3} \cap F_{\infty}, E_{\infty, 3} \cap E_{\infty, 1}$ and $E_{\infty, 3} \cap E_{\infty, 2}$, respectively. Then we have Figure 7, in which we give the self-intersection numbers of the curves and the thick curves are integral with respect to $D$.

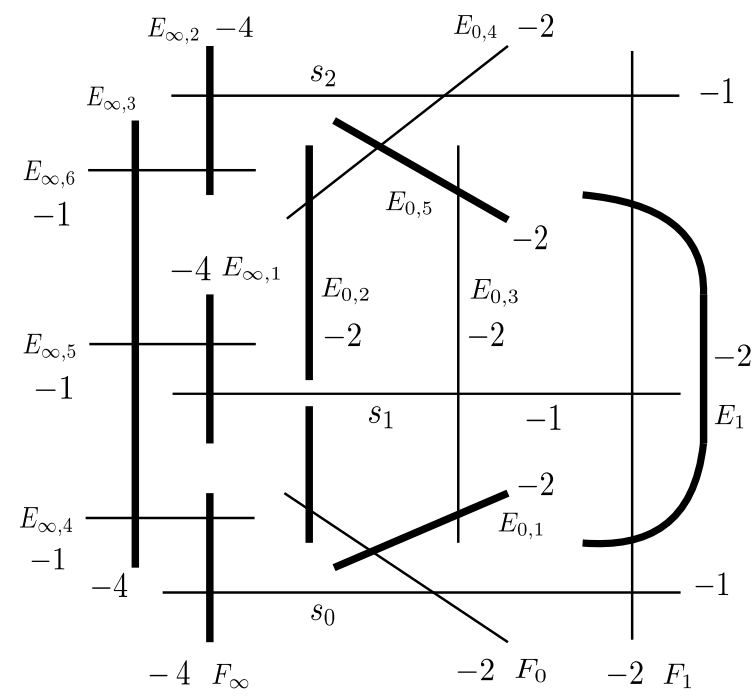

Figure 7

A direct calculation shows the following lemmas.

Lemma 7.11. (i) The divisorial part $(D)$ of $D$ on $Y$ is given by

$$
-\left(E_{1}+E_{0,1}+E_{0,2}+E_{0,5}+F_{\infty}+E_{\infty, 1}+E_{\infty, 2}\right)-2\left(E_{\infty, 3}+E_{\infty, 4}+E_{\infty, 5}+E_{\infty, 6}\right) .
$$

(ii) The integral curves in Figure 7 are $E_{0,1}, E_{0,2}, E_{0,5}, F_{\infty}, E_{\infty, 1}, E_{\infty, 2}, E_{\infty, 3}, E_{1}$.

Lemma 7.12. (i) $(D)^{2}=-12$.

(ii) The canonical divisor $K_{Y}$ of $Y$ is given by

$$
K_{Y}=-\left(F_{\infty}+E_{\infty, 1}+E_{\infty, 2}\right)-2\left(E_{\infty, 3}+E_{\infty, 4}+E_{\infty, 5}+E_{\infty, 6}\right) .
$$

(iii) $K_{Y} \cdot(D)=-4$.

Now, by taking the quotient by $D$, we have Figure 8 . Here, the numbers -1 and -4 denote the self-intersection numbers of curves. The other curves have self-intersection number -2 .

We now contract four $(-1)$-curves in Figure 8 and denote by $X_{a}$ the resulting surface, which has the dual graph of $(-2)$-curves given in Figure 5 (recall that the vector field (7.7) contains a parameter $a$ ). We use the notation of Figure 5 . On $X_{a}$, there exist exactly one elliptic fibration with singular fibers of type $\left(2 \mathrm{IV}^{*}, \mathrm{I}_{3}, \mathrm{I}_{1}\right)$, defined by the linear system $\left|E_{8}+E_{9}+E_{10}\right|$, and three quasi-elliptic fibrations with singular fibers of type (III*,2III), defined by $\left|2\left(E_{9}+E_{12}\right)\right|$, $\left|2\left(E_{8}+E_{11}\right)\right|$ and $\left|2\left(E_{10}+E_{13}\right)\right|$.

Theorem 7.13. The surfaces $\left\{X_{a}\right\}$ form a 1-dimensional non-isotrivial family of classical Enriques surfaces with dual graph given in Figure 5.

Proof. By using Lemmas 7.11 and 7.12 and the same argument as in the case of the supersingular surface in the previous subsection, we see that $X_{a}$ is an Enriques surface. Since the image of $F_{a}$ and the singular fiber of type $\mathrm{IV}^{*}$ are double fibers, $X_{a}$ is classical by Proposition 2.5. Moreover, 


\section{T. Katsura, S. Kondō And G. Martin}

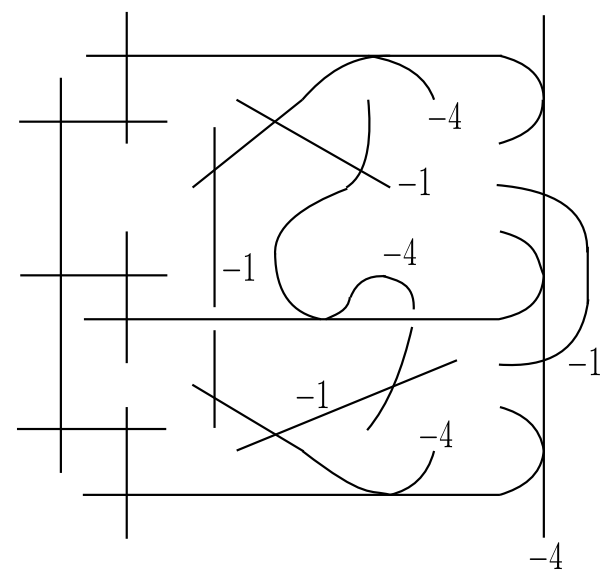

Figure 8

since the fibration we used to construct $X_{a}$ is not isotrivial, the $j$-invariant of the double fiber $F_{a}$ varies, and hence the family $\left\{X_{a}\right\}$ is non-isotrivial. By the same proof as that of Theorem 7.8, we prove that $X_{a}$ contains exactly thirteen $(-2)$-curves whose dual graph is given in Figure 5 .

Lemma 7.14. The map $\rho_{n}: \operatorname{Aut}\left(X_{a}\right) \rightarrow \mathrm{O}\left(\operatorname{Num}\left(X_{a}\right)\right)$ is injective.

Proof. Let $g \in \operatorname{Ker}\left(\rho_{n}\right)$. Then $g$ preserves each of the thirteen curves $E_{1}, \ldots, E_{13}$ (see Figure 5 ). First, note that $g$ fixes three points on each of $E_{8}, E_{9}, E_{10}$ (in contrast to the supersingular case, where only two distinct points are fixed). Hence $g$ fixes $E_{8}, E_{9}$ and $E_{10}$ pointwise. Let $p$ be the quasi-elliptic fibration with singular fibers of type (III*,2III) defined by the linear system $\left|2\left(E_{8}+E_{11}\right)\right|$, and let $F$ be a general fiber of $p$. The two curves $E_{9}$ and $E_{10}$ are 2 -sections of the fibration $p$. Then, $g$ fixes at least three points on $F$, namely the intersection points with $E_{9}$ and $E_{10}$ and the cusp of $F$. Hence $g$ fixes $F$ pointwise. Thus $\rho_{n}$ is injective.

By the same arguments as in Theorems 7.8 and 7.9, we now have the following theorem.

Theorem 7.15. The automorphism group $\operatorname{Aut}\left(X_{a}\right)$ is isomorphic to the symmetric group $\mathfrak{S}_{3}$ of degree 3 , and $X_{a}$ contains exactly thirteen (-2)-curves.

Proof. By Lemma 7.14, the group $\operatorname{Aut}\left(X_{a}\right)$ is a subgroup of the symmetry group of the dual graph of $(-2)$-curves which is isomorphic to $\mathfrak{S}_{3}$. By considering the actions of the Mordell-Weil groups of the Jacobian fibrations of genus 1 fibrations on $X_{a}$, we see that any symmetry of the dual graph can be realized by an automorphism of $X_{a}$.

\section{Enriques surfaces of type VII}

The first and the second author proved the following theorem based on a method given in [KK15].

THEOREM 8.1 ([KK18]). There exists a 1-dimensional non-isotrivial family of Enriques surfaces with dual graph of (-2)-curves given in Figure 9. A general member of this family is classical and one special member is supersingular. The automorphism group of any member in this family is isomorphic to the symmetric group $\mathfrak{S}_{5}$ of degree 5. The canonical cover of any member in this family has twelve ordinary nodes, and its minimal resolution is the supersingular $K 3$ surface with Artin invariant 1. 


\section{Classification of Enriques surfaces}

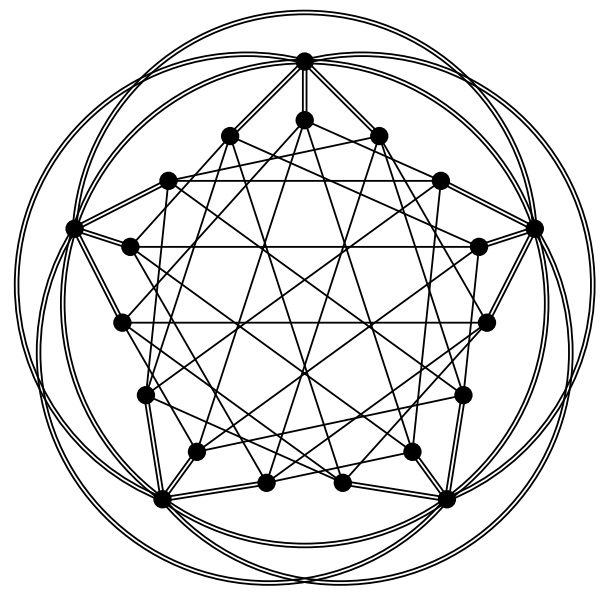

Figure 9

There exist elliptic fibrations with singular fibers of type $\left(\mathrm{I}_{9}, \mathrm{I}_{1}, \mathrm{I}_{1}, \mathrm{I}_{1}\right),\left(\mathrm{I}_{5}, \mathrm{I}_{5}, \mathrm{I}_{1}, \mathrm{I}_{1}\right),\left(\mathrm{I}_{8}, 2 \mathrm{III}\right)$ or $\left(\mathrm{I}_{6}, 2 \mathrm{IV}, \mathrm{I}_{2}\right)$ on Enriques surfaces of type VII. For more details, we refer the reader to [KK18].

\section{Enriques surfaces of type VIII}

In this section, we give a construction of a 1-dimensional family of classical Enriques surfaces with dual graph of type VIII.

We consider the relatively minimal non-singular complete elliptic surface $\psi: R \rightarrow \mathbf{P}^{1}$ associated with the Weierstrass equation

$$
y^{2}+s x y=x^{3}+s^{2} x
$$

with a parameter $s$. This surface is a rational elliptic surface with a singular fiber of type $I_{1}^{*}$ over the point given by $s=0$ and a singular fiber of type $\mathrm{I}_{4}$ over the point given by $s=\infty$ (Lang [Lan94, Section 2]). We consider the base change of $\psi: R \rightarrow \mathbf{P}^{1}$ by $s=t^{2}$. Then, we have the Weierstrass model defined by

$$
y^{2}+t x y+t y=x^{3}+x^{2}
$$

(Lang [Lan94, Section 2]). We consider the relatively minimal non-singular complete model of this elliptic surface:

$$
f: \tilde{R} \longrightarrow \mathbf{P}^{1} \text {. }
$$

The rational elliptic surface $f: \tilde{R} \rightarrow \mathbf{P}^{1}$ has a singular fiber of type III over the point given by $t=0$ and a singular fiber of type $\mathrm{I}_{8}$ over the point given by $t=\infty$.

On the singular elliptic surface (9.1), we denote by $F_{0}$ the fiber over the point defined by $t=0$ and by $E_{0}$ the fiber over the point defined by $t=\infty$. Both $F_{0}$ and $E_{0}$ are irreducible, and on each of $F_{0}$ and $E_{0}$, the surface (9.1) has only one singular point, respectively $P_{0}$ and $P_{\infty}$. The surface $\tilde{R}$ is a surface obtained by the minimal resolution of singularities of (9.1). We use the same symbol for the proper transforms of curves on $\tilde{R}$. The blow-up at the singular point $P_{0}$ gives one exceptional curve $F_{1}$, and the surface is non-singular along $F_{0}$ and $F_{1}$. The two curves $F_{1}$ and $F_{0}$ form a singular fiber of type III of the elliptic surface $f: \tilde{R} \rightarrow \mathbf{P}^{1}$. On the other hand, the blow-up at the singular point $P_{\infty}$ gives two exceptional curves $E_{1}$ and $E_{2}$, and the surface is non-singular along $E_{0}$ and has a unique singular point $P_{1}$, which is the intersection of $E_{1}$ and $E_{2}$. 


\section{T. Katsura, S. Kondō And G. Martin}

The blow-up at the singular point $P_{1}$ gives two exceptional curves $E_{3}$ and $E_{4}$. The curves $E_{3}$ and $E_{4}$ meet at one point $P_{2}$, which is a singular point of the resulting surface. The blow-up at the singular point $P_{2}$ again gives two exceptional curves $E_{5}$ and $E_{6}$. The curves $E_{5}$ and $E_{6}$ meet at one point $P_{3}$, which is a singular point of the resulting surface. Finally, the blow-up at the singular point $P_{3}$ gives an exceptional curve $E_{7}$, and the resulting surface is non-singular over these curves. The cycle

$$
E_{0}+E_{1}+E_{2}+E_{3}+E_{4}+E_{5}+E_{6}+E_{7}
$$

forms a singular fiber of type $\mathrm{I}_{8}$ given in Figure 10.

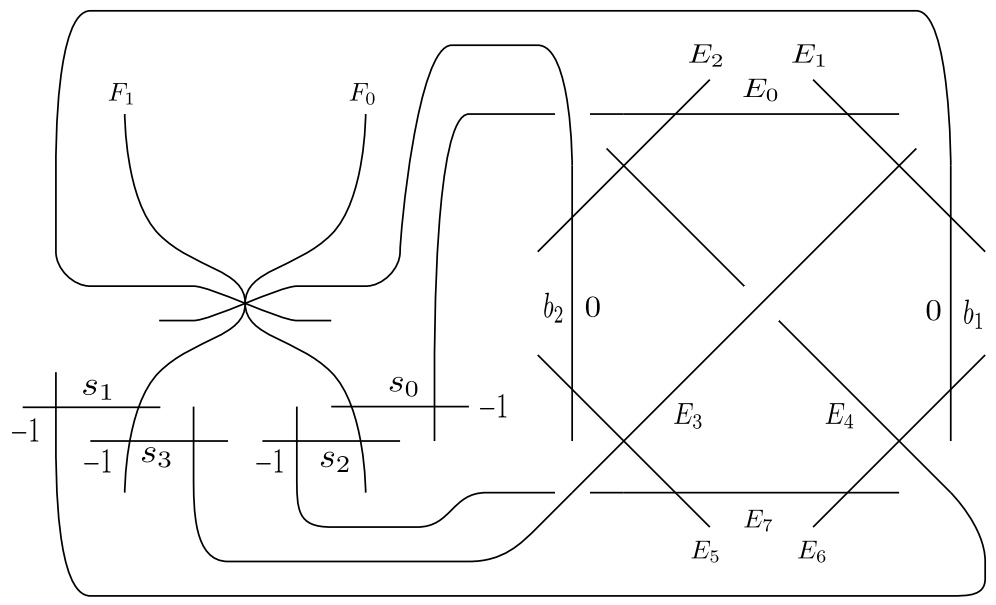

Figure 10

The elliptic surface $f: \tilde{R} \rightarrow \mathbf{P}^{1}$ has four sections $s_{i}(i=0,1,2,3)$ given as follows:

$s_{0}$ : the zero section, $s_{1}: x=y=0, s_{2}: x=t, y=0, s_{3}: x=0, y=t$.

Also, we consider the following two 2 -sections $b_{1}$ and $b_{2}$ defined by

$$
\begin{aligned}
& b_{1}: x+y=0, \quad x^{2}+t x+t=0, \\
& b_{2}: x+y+t x+t=0, \quad x^{2}+t x+t=0 .
\end{aligned}
$$

The configuration of singular fibers, four sections and two 2-sections is given in Figure 10.

Now, we consider a rational vector field on $\tilde{R}$ defined by

$$
D=D_{a}=t(a t+1) \frac{\partial}{\partial t}+(x+1) \frac{\partial}{\partial x}, \quad 0 \neq a \in k .
$$

Then, we have $D^{2}=D$; that is, $D$ is 2-closed. However, $D$ has an isolated singularity at the point $P$ which is the singular point of the fiber of type III, that is, the intersection point of two curves $F_{0}$ and $F_{1}$ (note that $(x, t)$ is not a local parameter along $F_{0}$ ). To resolve this singularity, we first blow up at $P$. Denote by $F_{2}$ the exceptional curve. We denote the proper transforms of $F_{0}$ and $F_{1}$ by the same symbols. Then the induced vector field has three isolated singularities, one of which is the intersection of three curves and the other two of which lie on the curve $F_{2}$. Blow up at these three points. Let $Y$ be the resulting surface and $\psi: Y \rightarrow \tilde{R}$ the successive blow-ups. We denote the induced vector field by the same symbol $D$ and the four exceptional curves by $F_{2}, F_{3}, F_{4}, F_{5}$. Then we have Figure 11 .

In Figure 11, we give the self-intersection numbers of the curves except for the curves with selfintersection number -2 . The thick lines are integral curves with respect to $D$. Denote by $F_{a}$ the 


\section{Classification of Enriques surfaces}

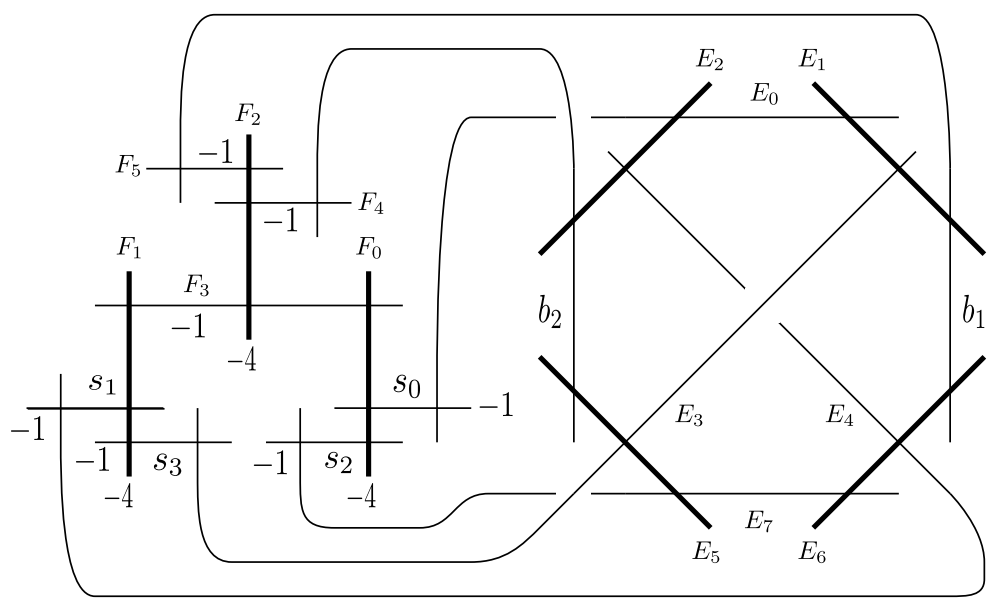

Figure 11

fiber over the point defined by $t=a^{-1}$. Then $F_{a}$ is integral with respect to $D$. Now, according to the above blow-ups, we have the following lemmas.

Lemma 9.1. (i) The divisorial part $(D)$ of the vector field $D$ on $Y$ is given by

$$
-\left(F_{0}+F_{1}+F_{2}+2 F_{3}+E_{1}+E_{2}+E_{5}+E_{6}\right) .
$$

(ii) The integral curves in Figure 11 are $F_{0}, F_{1}, F_{2}, E_{1}, E_{2}, E_{5}, E_{6}$,

LEMMA 9.2. (i) $(D)^{2}=-12$.

(ii) The canonical divisor $K_{Y}$ of $Y$ is given by $K_{Y}=-\left(F_{0}+F_{1}+F_{2}+2 F_{3}\right)$.

(iii) $K_{Y} \cdot(D)=-4$.

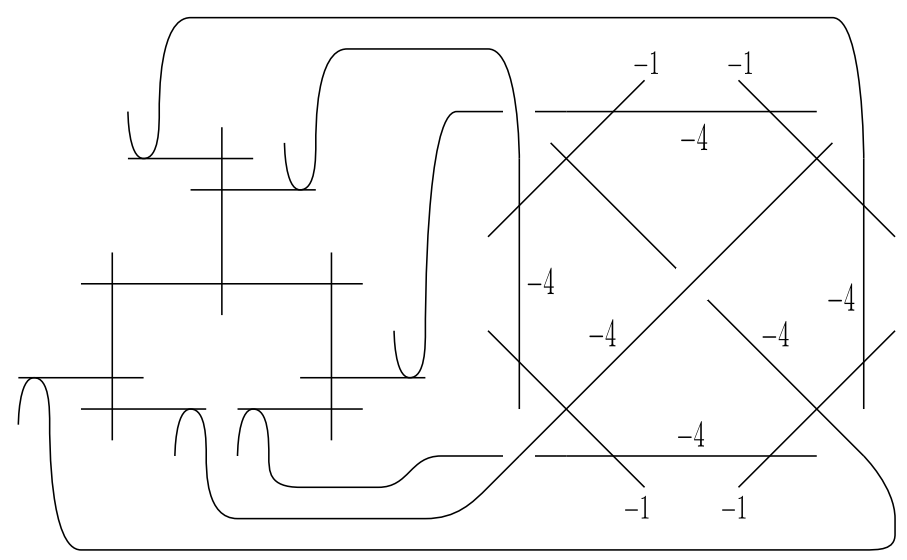

Figure 12

Now take the quotient $Y^{D}$ of $Y$ by $D$. By using the same argument as in the proof of Lemma 7.3, we see that $D$ is divisorial and $Y^{D}$ is non-singular. By Proposition 2.1, we have the configuration of curves in Figure 12. In that figure, we give the self-intersection numbers of the curves except for the curves with self-intersection number -2 .

Let $X_{a}$ be the surface obtained by contracting the four exceptional curves in Figure 12 (recall 


\section{T. Katsura, S. Kondō And G. Martin}

that the vector field $D$ contains a parameter $a)$. Then we have the configuration of $(-2)$-curves in Figure 13.

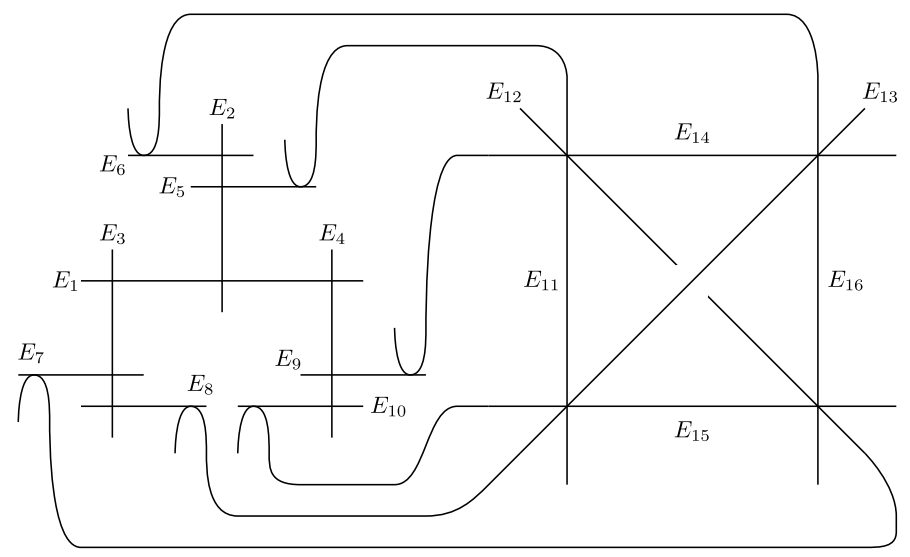

Figure 13

The dual graph of the sixteen $(-2)$-curves in Figure 13 is nothing but the one given in Figure 14. Note that any maximal parabolic subdiagram of this diagram is of type $\tilde{D}_{5} \oplus \tilde{A}_{3}$, $\tilde{D}_{6} \oplus \tilde{A}_{1} \oplus \tilde{A}_{1}$ or $\tilde{E}_{6} \oplus \tilde{A}_{2}$. On $X_{a}$, there are three types of genus 1 fibrations: three elliptic fibrations with singular fibers of type $\left(2 \mathrm{I}_{1}^{*}, \mathrm{I}_{4}\right)$, three quasi-elliptic fibrations with singular fibers of type ( $\left.\mathrm{I}_{2}^{*}, 2 \mathrm{III}, 2 \mathrm{III}\right)$ and eight elliptic fibrations with singular fibers of type $\left(\mathrm{IV}^{*}, \mathrm{I}_{3}, \mathrm{I}_{1}\right)$.

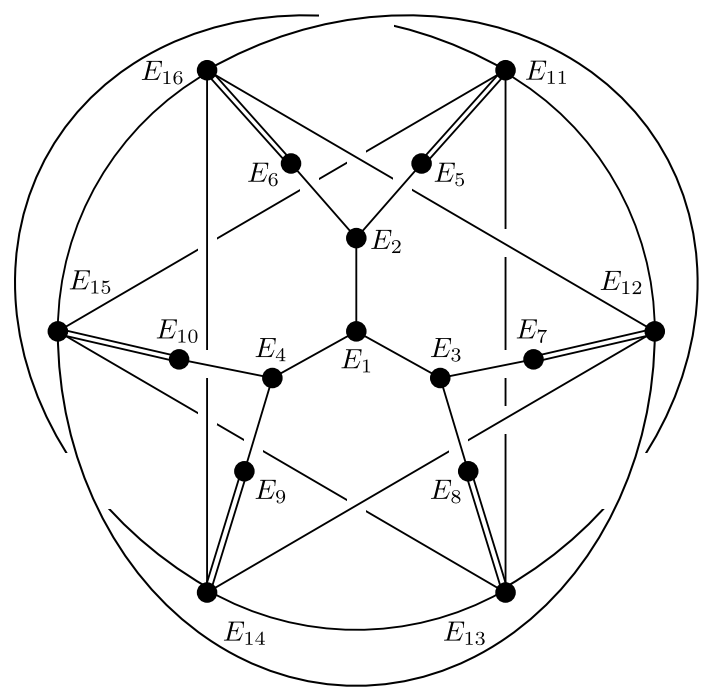

Figure 14

Theorem 9.3. The surfaces $\left\{X_{a}\right\}$ form a non-isotrivial 1-dimensional family of classical Enriques surfaces with dual graph given in Figure 14.

Proof. By using Lemmas 9.1 and 9.2 and the same argument as in the proof of Theorem 7.7, we see that $X_{a}$ is an Enriques surface. Since $X_{a}$ has a quasi-elliptic fibration defined by $\mid 2\left(E_{5}+\right.$ $\left.E_{11}\right)|=| 2\left(E_{6}+E_{16}\right) \mid$ with two double fibers, $X_{a}$ is classical (Proposition 2.5). Note that the image of $F_{a}$ is a double fiber of an elliptic fibration with singular fibers of type $\left(2 \mathrm{I}_{1}^{*}, \mathrm{I}_{4}\right)$. Since the fibration is non-isotrivial, the $j$-invariant of $F_{a}$ varies, and hence the family $\left\{X_{a}\right\}$ is non-isotrivial. 


\section{Classification of Enriques surfaces}

By the same proof as that of Theorem 7.8 , we see that $X_{a}$ contains exactly sixteen $(-2)$-curves, whose dual graph is given in Figure 14.

Theorem 9.4. The automorphism group $\operatorname{Aut}\left(X_{a}\right)$ is isomorphic to $\mathfrak{S}_{4}$.

Proof. The quasi-elliptic fibration defined by $\left|2\left(E_{5}+E_{11}\right)\right|$ has five 2-sections $E_{2}, E_{12}, E_{13}$, $E_{14}, E_{15}$. Each of these 2-sections meets other $(-2)$-curves at three different points, and hence they are fixed by any numerically trivial automorphism. Therefore, by the same proof as that of Lemma 7.14, the natural map $\rho_{n}: \operatorname{Aut}\left(X_{a}\right) \rightarrow \mathrm{O}\left(\operatorname{Num}\left(X_{a}\right)\right)$ is injective. Note that the automorphism group of the dual graph is isomorphic to the symmetric group $\mathfrak{S}_{4}$. By considering the actions of the Mordell-Weil groups of the Jacobian fibrations of genus 1 fibrations on $X_{a}$, we have proved that $\operatorname{Aut}\left(X_{a}\right) \cong \mathfrak{S}_{4}$.

\section{Enriques surfaces of type $\tilde{E}_{8}$}

In this section, we give constructions of supersingular and classical Enriques surfaces with dual graph of all (-2)-curves given in Figure 15.

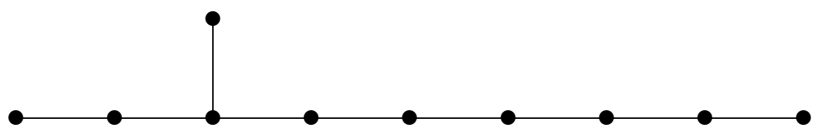

Figure 15

\subsection{Supersingular case}

Let $(x, y)$ be affine coordinates of $\mathbf{A}^{2} \subset \mathbf{P}^{2}$. Consider a rational vector field $D$ defined by

$$
D=\frac{1}{x^{5}}\left(\left(x y^{6}+x^{3}\right) \frac{\partial}{\partial x}+\left(x^{6}+y^{7}+x^{2} y\right) \frac{\partial}{\partial y}\right) .
$$

Then $D^{2}=0$; that is, $D$ is 2-closed. Note that $D$ has a pole of order 5 along the line $\ell$ defined by $x=0$ and that this line is integral with respect to $D$. We see that $D$ has a unique isolated singularity $(x, y)=(0,0)$. First blow up at the point $(0,0)$. Then we see that the exceptional curve is not integral and that the induced vector field has a pole of order 2 along the exceptional curve. Moreover, the induced vector field has a unique isolated singularity at the intersection of the proper transform of $\ell$ and the exceptional curve. Continue this process until the induced vector field has no isolated singularities. The final configuration of curves is given in Figure 16. Here, $F_{0}$ is the proper transform of $\ell$, and the suffix $i$ of the exceptional curve $E_{i}$ corresponds to the order of the successive blow-ups.

We denote by $Y$ the surface obtained by this process. We denote by the same symbol $D$ the induced vector field on $Y$. By direct calculations, we have the following lemmas.

Lemma 10.1. (i) The integral curves with respect to $D$ in Figure 16 are all horizontal curves (thick lines).

(ii) We have

$$
\begin{aligned}
(D)=- & \left(5 F_{0}+2 E_{1}+6 E_{2}+8 E_{3}+7 E_{4}+4 E_{5}+3 E_{6}+2 E_{7}+4 E_{8}+5 E_{9}\right. \\
& \left.+6 E_{10}+8 E_{11}+4 E_{12}+6 E_{13}\right) .
\end{aligned}
$$


T. Katsura, S. Kondō And G. Martin

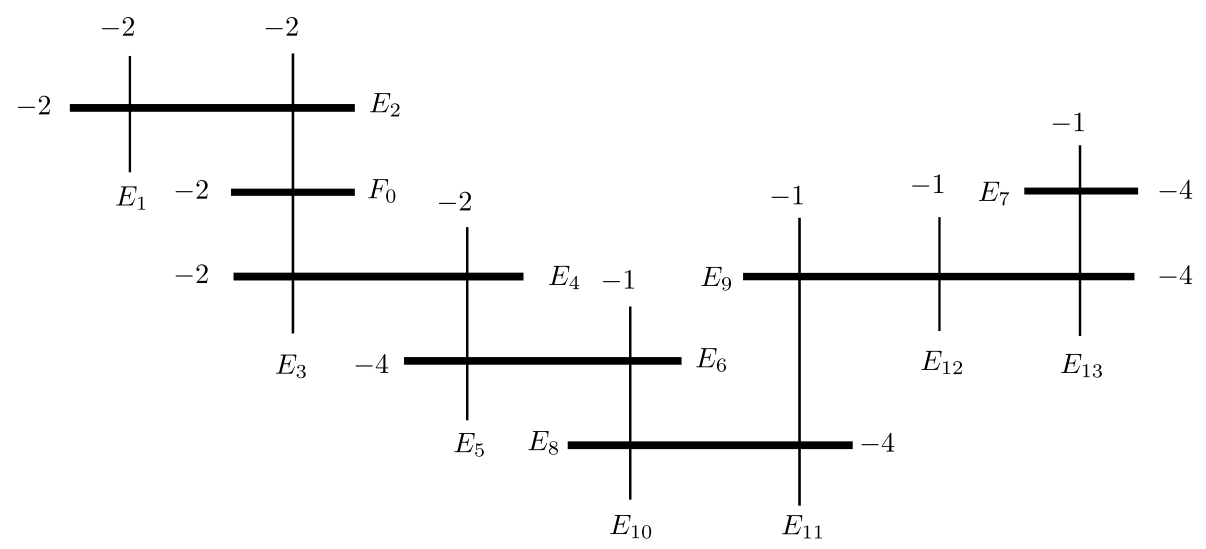

Figure 16

Lemma 10.2. (i) $(D)^{2}=-12$.

(ii) The canonical divisor $K_{Y}$ of $Y$ is given by

$$
\begin{aligned}
K_{Y}=- & \left(3 F_{0}+2 E_{1}+4 E_{2}+6 E_{3}+5 E_{4}+4 E_{5}+3 E_{6}+2 E_{7}+4 E_{8}+5 E_{9}\right. \\
& \left.+6 E_{10}+8 E_{11}+4 E_{12}+6 E_{13}\right) .
\end{aligned}
$$

(iii) $K_{Y} \cdot(D)=-4$.

Now take the quotient $Y^{D}$ of $Y$ by $D$. By using the same argument as in the proof of Lemma 7.3, we see that $D$ is divisorial, and hence $Y^{D}$ is non-singular. By Proposition 2.1, we have the configuration of curves on $Y^{D}$ in Figure 17.

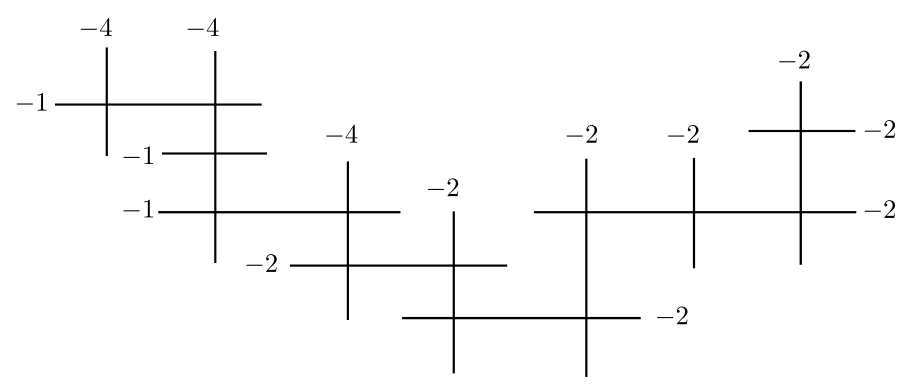

Figure 17

By contracting the three exceptional curves, we get a new exceptional curve which is the image of the (-4)-curve meeting the three exceptional curves. Let $X$ be the surface obtained by contracting the exceptional curve. The surface $X$ contains ten $(-2)$-curves whose dual graph is given by Figure 15. Note that this diagram contains a unique maximal parabolic subdiagram, which is of type $\tilde{E}_{8}$. The pencil of lines in $\mathbf{P}^{2}$ through $(x, y)=(0,0)$ induces a quasi-elliptic fibration on $X$ with a double fiber of type $\mathrm{II}^{*}$ (the fibration is quasi-elliptic since it is dominated by a pencil of lines).

TheOREm 10.3. The surface $X$ is a supersingular Enriques surface with dual graph given in Figure 15.

Proof. By using Lemmas 10.1 and 10.2 and the same arguments as in the proofs of Theorems 7.7 and 7.8, we see that $X$ is an Enriques surface with dual graph given in Figure 15. Note that 


\section{Classification of EnRiques surfaces}

the normalization of the canonical cover of $X$ is obtained from $Y$ by contracting the divisor $F_{0}+E_{2}+E_{3}+E_{4}$, and hence it has a rational double point of type $D_{4}$. It follows from Lemma 3.7 that $X$ is supersingular.

Theorem 10.4. Aut $(X)=\operatorname{Aut}_{n t}(X)=\operatorname{Aut}_{\mathrm{ct}}(X) \cong \mathbf{Z} / 11 \mathbf{Z}$.

Proof. First note that the dual graph has no symmetries and hence $\operatorname{Aut}(X)=\operatorname{Aut}_{\mathrm{nt}}(X)$. Since $X$ is supersingular, $\operatorname{Aut}_{\mathrm{ct}}(X)=\operatorname{Aut}_{\mathrm{nt}}(X)$.

Now we consider the vector field (10.1), and we set $u=x^{2}, v=y^{2}, z=x^{7}+x y^{7}+x^{3} y$. Then, we have $D(u)=0, D(v)=0, D(z)=0$ and the equation

$$
z^{2}=u^{7}+u v^{7}+u^{3} v .
$$

Therefore, the quotient surface of $\mathbf{P}^{2}$ by $D$ is birational to the surface defined by (10.2), which is birational to our Enriques surface. To do a change of coordinates, we define new variables $x, y, t$ by $x=1 / u, y=z / u^{4}$ and $t=v / u$. Then, the equation becomes

$$
y^{2}+t x^{4}+x+t^{7}=0 .
$$

This equation gives a non-singular affine chart of a quasi-elliptic surface $f: X \rightarrow \mathbf{P}^{1}$ by sending $(x, y, t)$ to $t$. Set

$$
A=k[t, x, y] /\left(y^{2}+t x^{4}+x+t^{7}\right),
$$

and let $\sigma$ be an automorphism of our Enriques surface. The double fiber of $f$ of type II $^{*}$, denoted by $2 F_{\infty}$, exists over the point defined by $t=\infty$. Since $\sigma$ preserves the diagram of $(-2)$-curves, $\sigma$ preserves the curve $C$ of cusps and $2 F_{\infty}$. Therefore, $\sigma$ has the form given in (6.4).

Together with the equation (10.3), we have an identity

$$
\begin{aligned}
& e_{1}(t, x)^{2}\left(t x^{4}+x+t^{7}\right)+e_{2}(t, x)^{2} \\
& \quad=\left(c_{1} t+c_{2}\right)\left(d_{1}(t) x+d_{2}(t)\right)^{4}+\left(d_{1}(t) x+d_{2}(t)\right)+\left(c_{1} t+c_{2}\right)^{7} .
\end{aligned}
$$

Using Lemma 6.3 and taking the coefficients of $x$, we have $e_{1}(t, x)^{2}+d_{1}(t)=0$. Hence $e_{1}(t, x)$ is a polynomial of $t$; that is, we can put $e_{1}(t, x)=e_{1}(t)$ and $d_{1}(t)=e_{1}(t)^{2}$. Taking the coefficients of $t$, we have $e_{1}(t)^{2} x^{4}+e_{1}(t)^{2} t^{6}+c_{1}\left(d_{1}(t) x+d_{2}(t)\right)^{4}+d_{2}(t)_{\text {odd }} / t+c_{1}\left(c_{1} t+c_{2}\right)^{6}=0$. Here, $d_{2}(t)_{\text {odd }}$ consists of the odd terms of $d_{2}(t)$. Considering the coefficients of $x^{4}$ of this equation, we have $e_{1}(t)^{2}=c_{1} d_{1}(t)^{4}=c_{1} e_{1}(t)^{8}$. Since we have $e_{1}(t) \not \equiv 0$, we have $e_{1}(t)^{6}=1 / c_{1}$. Therefore, $e_{1}(t)$ is a constant; we set $e_{1}(t)=e_{1} \in k$. Then, $e_{1}^{6}=1 / c_{1}$. Thus we have an identity $e_{1}^{2} t^{6}+c_{1} d_{2}(t)^{4}+$ $d_{2}(t)_{\text {odd }} / t+c_{1}\left(c_{1} t+c_{2}\right)^{6}=0$ with $e_{1}^{6}=1 / c_{1}$. Let $d_{2}(t)$ be of degree $m$. If $m \geqslant 2$, then we have $\operatorname{deg} d_{2}(t)^{4} \geqslant 8$, and we cannot kill the highest term of $d_{2}(t)^{4}$ in the equation. Therefore, we can put $d_{2}(t)=b_{0}+b_{1} t\left(b_{0}, b_{1} \in k\right)$, and we have an identity

$$
\left(e_{1}^{2}+c_{1}^{7}\right) t^{6}+\left(c_{1} b_{1}^{4}+c_{1}^{5} c_{2}^{2}\right) t^{4}+c_{1}^{3} c_{2}^{4} t^{2}+\left(c_{1} b_{0}^{4}+b_{1}+c_{1} c_{2}^{6}\right)=0 .
$$

Hence we have $e_{1}^{2}+c_{1}^{7}=0, c_{1} b_{1}^{4}+c_{1}^{5} c_{2}^{2}=0, c_{1}^{3} c_{2}^{4}=0$ and $c_{1} b_{0}^{4}+b_{1}+c_{1} c_{2}^{6}=0$ with $e_{1}^{6}=1 / c_{1}$. Since $c_{1} \neq 0$, we have $c_{2}=b_{1}=b_{0}=0$ and $c_{1}=\zeta, e_{1}=\zeta^{9}, d_{1}=\zeta^{7}$ with $\zeta^{11}=1$. Putting these data into the original equation, we have $e_{2}(t, x)=0$. Thus we have

$$
\sigma(t)=\zeta t, \quad \sigma(x)=\zeta^{7} x, \quad \sigma(y)=\zeta^{9} y,
$$

and we conclude $\operatorname{Aut}(X) \cong \mathbf{Z} / 11 \mathbf{Z}$.

Remark 10.5. The numerically trivial automorphism $\sigma$ of order 11 found here leads to one of the exceptions in [DM19]. 


\section{T. Katsura, S. Kondō And G. Martin}

\subsection{Classical case}

Let $Q=\mathbf{P}^{1} \times \mathbf{P}^{1}$ be a non-singular quadric, and let $\left(\left(u_{0}, u_{1}\right),\left(v_{0}, v_{1}\right)\right)$ be homogeneous coordinates of $Q$. Let $x=u_{0} / u_{1}$ and $y=v_{0} / v_{1}$. Consider a rational vector field $D$ defined by

$$
D=\frac{1}{x^{3} y^{2}}\left(x^{4} y^{2} \frac{\partial}{\partial x}+\left(x^{2}+a x^{4} y^{4}+y^{4}\right) \frac{\partial}{\partial y}\right), \quad a \in k^{*} .
$$

Then $D^{2}=D$; that is, $D$ is 2-closed. Note that $D$ has a pole of order 3 along the divisor defined by $x=0$, a pole of order 1 along the divisor defined by $x=\infty$ and a pole of order 2 along the divisor defined by $y=0$. Moreover, $D$ has two isolated singularities at $(x, y)=(0,0),(\infty, 0)$. As in the case of supersingular Enriques surfaces of type $E_{8}$, we repeatedly blow up the points of isolated singularities of $D$ and finally get a vector field $D$, denoted by the same symbol, without isolated singularities. The configuration of curves is given in Figure 18 . Here, $F_{0}, E_{1}$ and $E_{2}$ are the proper transforms of the curves defined by $y=0, x=0$ and $x=\infty$, respectively, and the suffix $i$ of the other exceptional curve $E_{i}$ corresponds to the order of the successive blow-ups. We denote by $Y$ the surface obtained by these successive blow-ups.

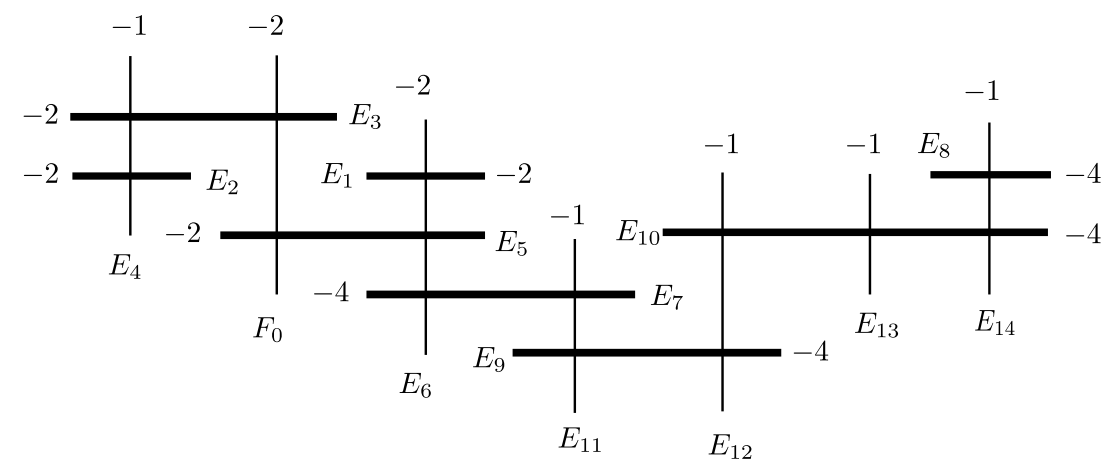

Figure 18

A direct calculation shows the following two lemmas.

Lemma 10.6. (i) The integral curves with respect to $D$ in Figure 18 are all horizontal curves (thick lines).

(ii)

$$
\begin{aligned}
(D)=- & \left(2 F_{0}+3 E_{1}+E_{2}+2 E_{3}+4 E_{5}+4 E_{6}+3 E_{7}+2 E_{8}+4 E_{9}+5 E_{10}\right. \\
& \left.+6 E_{11}+8 E_{12}+4 E_{13}+6 E_{14}\right) .
\end{aligned}
$$

LEMMA 10.7. (i) $(D)^{2}=-12$.

(ii) The canonical divisor $K_{Y}$ of $Y$ is given by

$$
\begin{aligned}
K_{Y}=- & \left(2 F_{0}+2 E_{1}+E_{3}+3 E_{5}+4 E_{6}+3 E_{7}+2 E_{8}+4 E_{9}+5 E_{10}\right. \\
& \left.+6 E_{11}+8 E_{12}+4 E_{13}+6 E_{14}\right) .
\end{aligned}
$$

(iii) $K_{Y} \cdot(D)=-4$.

Now take the quotient $Y^{D}$ of $Y$ by $D$. By using the same argument as in the proof of Lemma 7.3, we see that $Y^{D}$ is non-singular. By Proposition 2.1, we have the configuration of curves in Figure 19. 
Classification of Enriques surfaces

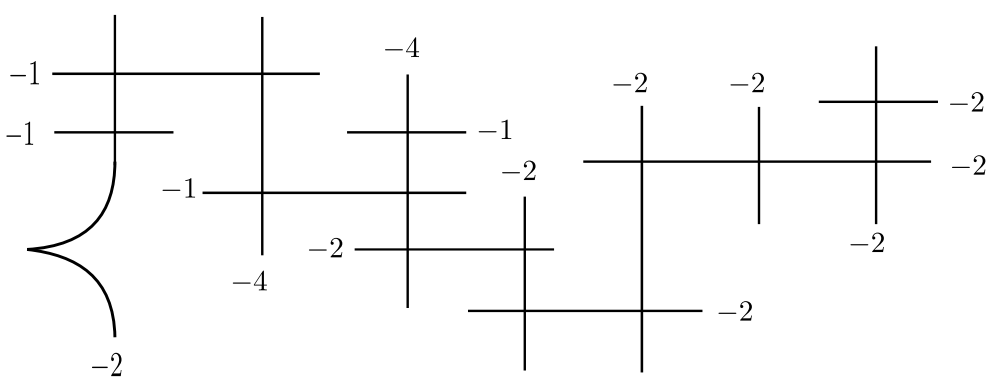

Figure 19

Let $X_{a}$ be the surface obtained by contracting the four exceptional curves in Figure 19 (recall that the vector field $D$ contains one parameter $a$; see (10.4)). Then $X_{a}$ contains ten $(-2)$-curves, whose dual graph is given by Figure 15. Recall that this diagram contains a unique maximal parabolic subdiagram which is of type $\tilde{E}_{8}$. The first projection from $Q$ to $\mathbf{P}^{1}$ is a $\mathbf{P}^{1}$ bundle, and it induces a quasi-elliptic fibration on $X_{a}$ with two double fibers of type II* and of type II.

Theorem 10.8. The surfaces $\left\{X_{a}\right\}$ form a 1-dimensional family of classical Enriques surfaces with dual graph given in Figure 15.

Proof. By using Lemmas 10.6 and 10.7 and the same arguments as in the proofs of Theorems 7.7 and 7.8, we see that $X_{a}$ is an Enriques surface with dual graph given in Figure 15. Since $X_{a}$ has a genus 1 fibration with two double fibers of type II $^{*}$ and II, the surface $X_{a}$ is classical (Proposition 2.5).

Theorem 10.9. The automorphism group $\operatorname{Aut}\left(X_{a}\right)$ is trivial.

Proof. We consider the vector field (8.2), and we set $u=x^{2}, v=y^{2}, z=x^{3}+a x^{5} y^{4}+x y^{4}+x^{4} y^{3}$. Then, we have $D(u)=0, D(v)=0, D(z)=0$ and the equation

$$
z^{2}=u^{3}+a^{2} u^{5} v^{4}+u v^{4}+u^{4} v^{3}, \quad a \in k^{*} .
$$

Therefore, the quotient surface of $\mathbf{P}^{1} \times \mathbf{P}^{1}$ by $D$ is birational to the surface defined by (10.5), which is birational to our Enriques surface. To do a change of coordinates, we define new variables $x, y, t$ by

$$
x=1 / a^{3 / 4} u v, \quad y=z / a^{7 / 4} u^{4} v^{2}, \quad t=1 / \sqrt{a} u,
$$

and we replace $1 / a^{5 / 4}$ by $b$ for the sake of simplicity. Then, the equation becomes $y^{2}+t x^{4}+$ $b t^{3} x+t^{3}+t^{7}=0$. This equation gives a normal affine surface. Now by a calculation similar to the one in the proof of Theorem 10.4, we see that there are no non-trivial automorphisms; that is, $\operatorname{Aut}\left(X_{a}\right)$ is trivial.

\section{Enriques surfaces of type $\tilde{E}_{7}+{\tilde{A_{1}}}^{(1)}$ and $\tilde{E}_{7}+{\tilde{A_{1}}}^{(2)}$}

\subsection{Classical case of type $\tilde{E}_{7}+{\tilde{A_{1}}}^{(1)}$}

In this subsection, we give a construction of an Enriques surface with dual graph of all (-2)curves given in Figure 20.

Let $\left(X_{0}, X_{1}, X_{2}\right) \in \mathbf{P}^{2}$ and $(S, T) \in \mathbf{P}^{1}$ be homogeneous coordinates. Consider the surface $R$ in $\mathbf{P}^{2} \times \mathbf{P}^{1}$ defined by

$$
S\left(a X_{0}^{2}+b X_{2}^{2}\right)+T\left(X_{1}^{2}+a X_{1} X_{2}+b X_{0} X_{2}\right)=0, \quad a, b \in k^{*} .
$$




\section{T. Katsura, S. Kondō And G. Martin}

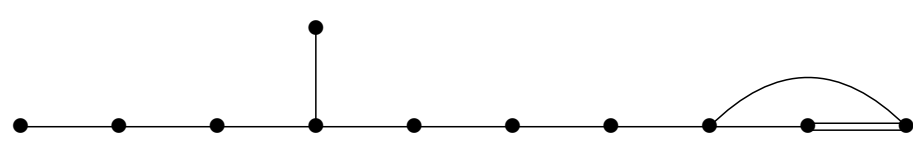

Figure 20

Note that the projection to $\mathbf{P}^{1}$ defines a fiber space $\pi: R \rightarrow \mathbf{P}^{1}$ whose general fiber is a nonsingular conic. Let $E_{1}$ be the fiber over the point $(S, T)=(0,1)$, which is non-singular. The fiber over the point $(S, T)=(1,0)$ is a double line denoted by $2 E_{2}$, and the fiber over the point $\left(b^{2}, a^{3}\right)$ is a union of two lines denoted by $E_{3}$ and $E_{4}$. The line defined by $X_{2}=0$ is a 2 -section, denoted by $F_{0}$, of the fiber space. The surface $R$ has two rational double points $Q_{i}=\left(\left(\alpha, \beta_{i}, 1\right),(1,0)\right)(i=1,2)$ of type $A_{1}$, where $\alpha=\sqrt{b / a}$ and the $\beta_{i}$ are the roots of the equation $y^{2}+a y+\sqrt{b^{3} / a}=0$.

Let $\left(x=X_{0} / X_{2}, y=X_{1} / X_{2}, s=S / T\right)$ be affine coordinates. Define

$$
D=\frac{1}{s}\left(a\left(s^{2}+c\right) \frac{\partial}{\partial x}+\left(a s^{2} x^{2}+b c\right) \frac{\partial}{\partial y}\right), \quad b \neq a^{2} c
$$

where $c$ is a root of the equation of $t^{2}+(b / a) t+1=0$. Then $D^{2}=a D$; that is, $D$ is 2-closed. A direct calculation shows that $D$ has two isolated singularities at the intersection points of $F_{0}$ and $E_{1}, E_{2}$. As in the case of supersingular Enriques surfaces of type $\tilde{E}_{8}$, we blow up the two rational double points and the points of isolated singularities of $D$ successively and finally get a vector field, denoted by the same symbol $D$, without isolated singularities. The configuration of curves is given in Figure 21. Here, the suffix $i$ of the exceptional curve $E_{i}$ corresponds to the order of the successive blow-ups.

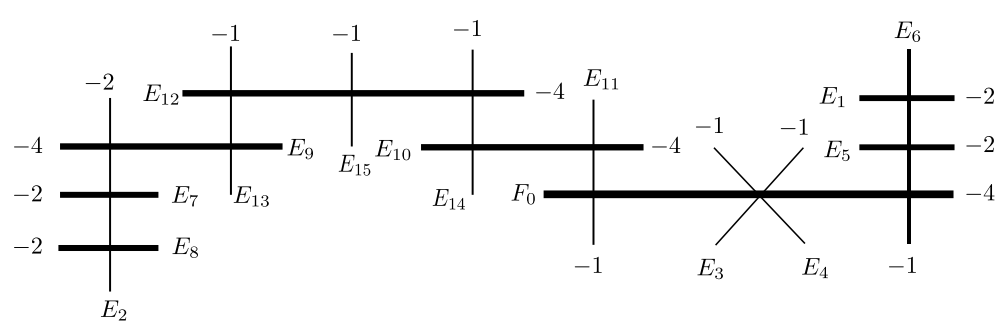

Figure 21

Now we denote by $Y$ the surface obtained by the successive blow-ups. By direct calculations, we have the following lemmas.

Lemma 11.1. (i) The integral curves with respect to $D$ in Figure 21 are all horizontal curves (thick lines).

(ii) $(D)=-\left(F_{0}+E_{1}+2 E_{2}+E_{5}+2 E_{7}+2 E_{8}+2 E_{9}+2 E_{10}+2 E_{11}+3 E_{12}+4 E_{13}+4 E_{14}+2 E_{15}\right)$.

Lemma 11.2. (i) $(D)^{2}=-12$.

(ii) The canonical divisor $K_{Y}$ of $Y$ is given by

$$
K_{Y}=-\left(F_{0}+2 E_{2}+E_{7}+E_{8}+2 E_{9}+2 E_{10}+2 E_{11}+3 E_{12}+4 E_{13}+4 E_{14}+2 E_{15}\right) .
$$

(iii) $K_{Y} \cdot(D)=-4$. 


\section{Classification of Enriques surfaces}

Now take the quotient $Y^{D}$ of $Y$ by $D$. By using the same argument as in the proof of Lemma 7.3, we see that $D$ is divisorial and hence $Y^{D}$ is non-singular. By Proposition 2.1, we have the configuration of curves in Figure 22.

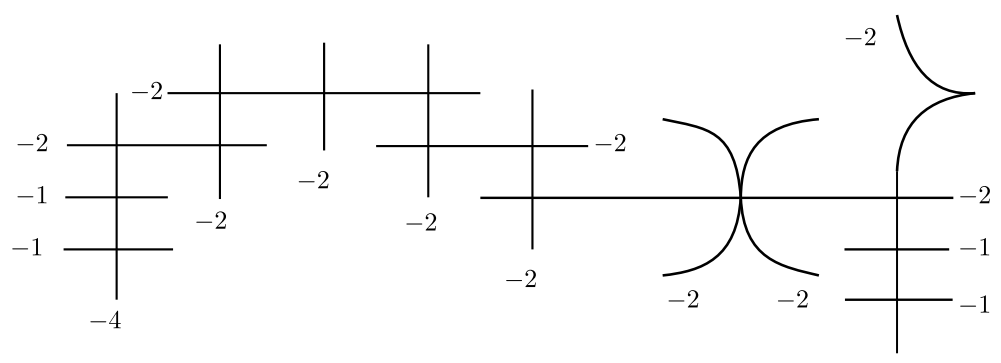

Figure 22

Let $X_{a, b}$ be the surface obtained by contracting the four exceptional curves. The surface $X_{a, b}$ contains eleven $(-2)$-curves, whose dual graph is given by Figure 20. Note that any maximal parabolic subdiagram of this diagram is of type $\tilde{E}_{7} \oplus \tilde{A}_{1}$ or $\tilde{E}_{8}$.

Theorem 11.3. The surfaces $\left\{X_{a, b}\right\}$ are classical Enriques surfaces with dual graph given in Figure 20. It contains an at least 1-dimensional non-isotrivial family.

Proof. By using Lemmas 11.1 and 11.2 and the same arguments as in the proofs of Theorems 7.7 and 7.8, we see that $X_{a, b}$ is an Enriques surface with dual graph given in Figure 20. Let $p_{1}$ be the genus 1 fibration on $X$ with a singular fiber III* induced from the fiber space $\pi: R \rightarrow \mathbb{P}^{1}$. By construction, $p_{1}$ has two double fibers (see Figure 22). Hence $X_{a, b}$ is classical (Proposition 2.5).

In Section 11.2, we construct classical Enriques surfaces with double fibers of type III* and III which are specializations of $\left\{X_{a, b}\right\}$. Note that the surface $X_{a, b}$ and the one given in the next subsection are not isomorphic because their dual graphs of all $(-2)$-curves are different (Figures 20 and 23). It follows from Matsusaka and Mumford [MM64, Theorem 1] that the family $\left\{X_{a, b}\right\}$ contains an at least 1-dimensional non-isotrivial family.

Before computing the automorphism group of $X_{a, b}$, let us summarize what we know about the genus 1 fibrations on $X_{a, b}$. Using Lemma 3.3, we easily see that the leftmost vertex in Figure 20 is part of the conductrix and hence, by Lemma 3.3 and Proposition 2.8, it is the curve of cusps of the two quasi-elliptic fibrations of type (II*). Then, similarly, Table 6 shows that the $\tilde{E}_{7}$ diagram is a double fiber of a quasi-elliptic fibration of type $\left(2 \mathrm{III}^{*}, \mathrm{III}\right)$, which is the fibration induced by $\pi$.

TheOREm 11.4. The automorphism group $\operatorname{Aut}\left(X_{a, b}\right)$ is $\mathbf{Z} / 2 \mathbf{Z}$; it is not numerically trivial.

Proof. First, note that it suffices to show that $X_{a, b}$ admits no numerically trivial automorphisms. Indeed, the symmetry group of the dual graph of (-2)-curves of $X_{a, b}$ is $\mathbf{Z} / 2 \mathbf{Z}$ (see Figure 20) and so is the Mordell-Weil group of the Jacobian fibration of $p_{1}$ (see [Ito94]). Since the Mordell-Weil group acts effectively on $X_{a, b}$ and it cannot act trivially on the graph, it realizes all of $\operatorname{Aut}\left(X_{a, b}\right)$.

So, let $g$ be a numerically trivial automorphism. Consider a quasi-elliptic fibration $p_{2}$ with a singular fiber of type II*. Let $C_{1}$ and $C_{2}$ be the double fibers of $p_{2}$, both of which are rational curves with a cusp, and let $C$ be the cuspidal double fiber of $p_{1}$. Note that $g$ preserves $C$ and fixes at least two points on it, namely the cusp of $C$ and the intersection of $C$ and the curve of cusps of $p_{1}$. If $g$ has odd order, then it also fixes $C_{i} \cap C$ since it will preserve the $C_{i}$. Hence by Lemma 2.13, the automorphism $g$ fixes $C$ pointwise. 


\section{T. Katsura, S. Kondō And G. Martin}

Since $C$ is a 2 -section of $p_{2}$, the double fiber $C_{i}$ is preserved by $g$. Thus, $g$ fixes three points on $C_{i}$, namely the cusp of $C_{i}$ and the intersection points of $C_{i}$ with the two double fibers of $p_{1}$. Hence again by Lemma 2.13, the automorphism $g$ fixes $C_{1}$ and $C_{2}$ pointwise. Similarly, $g$ fixes at least three points on a general fiber $F$ of $p_{1}$, namely its cusp and the intersection points with $C_{1}$ and $C_{2}$. Therefore, $g$ fixes $F$ pointwise. Thus $g$ is the identity.

\subsection{The case of type $\tilde{E}_{7}+{\tilde{A_{1}}}^{(2)}$}

In this subsection, we give a construction of classical Enriques surfaces with dual graph of all $(-2)$-curves given in Figure 23.

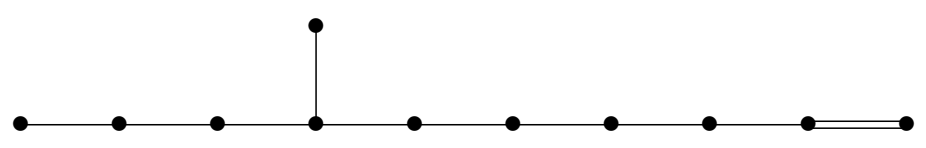

Figure 23

This example is a specialization of the previous example given in Section 11.1. In the equations (11.1) and (11.2), we set $b=0$ and then $c=1$. The fiber over the point $(S, T)=(0,1)$ is a union of two lines, denoted by $E_{1}$ and $E_{2}$, defined by $X_{1}\left(X_{1}+a X_{2}\right)=0$. The fiber over the point $(S, T)=(1,0)$ is a double line, denoted by $2 E_{3}$. The line defined by $X_{2}=0$, denoted by $F_{0}$, is a 2 -section of the fiber space. The surface $R$ has two rational double points $Q_{1}=((0,0,1),(1,0))$ and $Q_{2}=((0, a, 1),(1,0))$ of type $A_{1}$.

A direct calculation shows that $D$ has two isolated singularities at the intersection points of the 2 -section $F_{0}$ and the two fibers over the points $(S, T)=(1,0),(0,1)$. As in the previous case, we blow up the two rational double points and the points of isolated singularities of $D$ successively and finally get a vector field $D$, denoted by the same symbol, without isolated singularities. The configuration of curves is given in Figure 24.

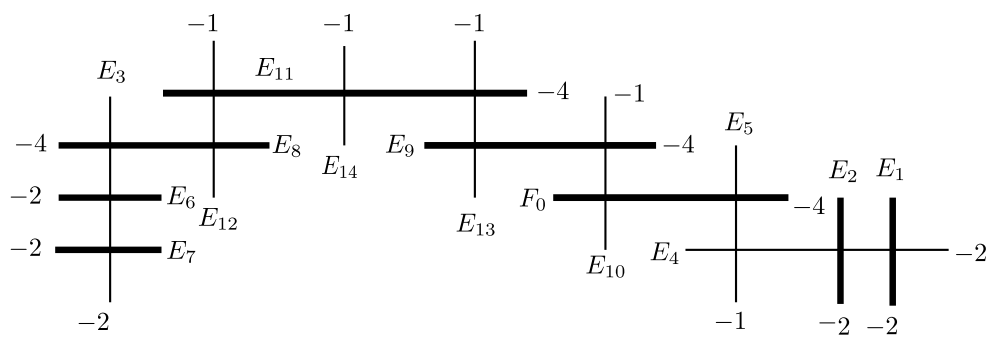

Figure 24

Here, we use the same symbols $F_{0}, E_{1}, E_{2}, E_{3}$ for the curves and their proper transforms, and the suffixes $i$ of the other exceptional curves $E_{i}$ correspond to the order of the successive blowups. The thick lines are integral curves. We denote by $Y$ the surface obtained by the successive blow-ups. By direct calculations, we have the following lemmas.

Lemma 11.5. (i) The integral curves with respect to $D$ in Figure 24 are $F_{0}, E_{1}, E_{2}, E_{6}, E_{7}, E_{8}$, $E_{9}, E_{11}$ (thick lines).

(ii) $(D)=-\left(F_{0}+E_{1}+E_{2}+2 E_{3}+2 E_{6}+2 E_{7}+2 E_{8}+2 E_{9}+2 E_{10}+3 E_{11}+4 E_{12}+4 E_{13}+2 E_{14}\right)$.

LeMma 11.6. (i) $(D)^{2}=-12$. 


\section{Classification of EnRiques surfaces}

(ii) The canonical divisor $K_{Y}$ of $Y$ is given by

$$
K_{Y}=-\left(F_{0}+2 E_{3}+E_{6}+E_{7}+2 E_{8}+2 E_{9}+2 E_{10}+3 E_{11}+4 E_{12}+4 E_{13}+2 E_{14}\right) .
$$

(iii) $K_{Y} \cdot(D)=-4$.

Now take the quotient $Y^{D}$ of $Y$ by $D$. By using the same argument as in the proof of Lemma 7.3, we see that $Y^{D}$ is non-singular. By Proposition 2.1, we have the configuration of curves in Figure 25.

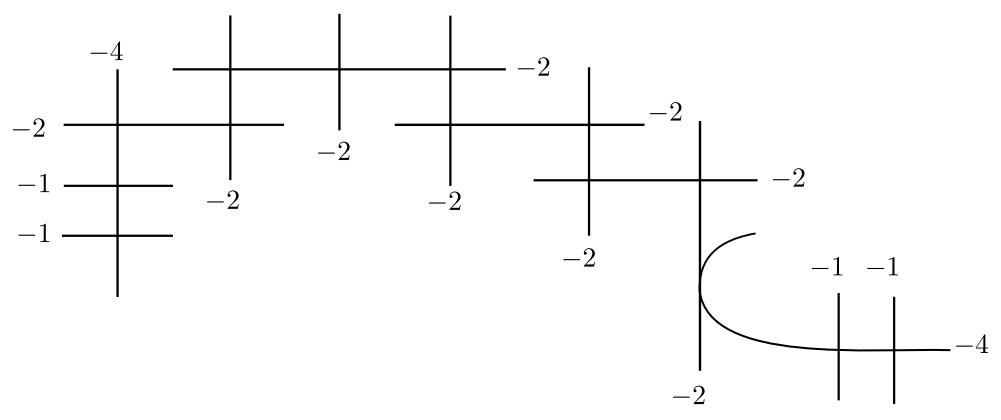

Figure 25

Let $X_{a}$ be the surface obtained by contracting the four exceptional curves. The surface $X_{a}$ contains eleven (-2)-curves, whose dual graph is given by Figure 23. Note that any maximal parabolic subdiagram of this diagram is of type $\tilde{E}_{7} \oplus \tilde{A}_{1}$ or $\tilde{E}_{8}$. Once we know that $X_{a}$ is an Enriques surface, an argument similar to the one in the previous subsection shows that the surface $X_{a}$ has a quasi-elliptic fibration of type (2III* 2 III) induced from the fiber space $\pi: R \rightarrow \mathbf{P}^{1}$ and a quasi-elliptic fibration of type $\left(\mathrm{II}^{*}\right)$.

Theorem 11.7. The surfaces $\left\{X_{a}\right\}$ form a 1-dimensional family of classical Enriques surfaces with dual graph given in Figure 23.

Proof. By using Lemmas 11.5 and 11.6 and the same arguments as in the proofs of Theorems 7.7 and 7.8, we see that $X_{a}$ is an Enriques surface with dual graph given in Figure 23. Since $X_{a}$ has a quasi-elliptic fibration with two double fibers, $X_{a}$ is classical (Proposition 2.5).

TheOREm 11.8. The automorphism group $\operatorname{Aut}\left(X_{a}\right)$ is $\mathbf{Z} / 2 \mathbf{Z}$; it is numerically trivial.

Proof. By an argument similar to the one for Theorem 11.4, we see that $\left|\operatorname{Aut}_{n t}\left(X_{a}\right)\right| \leqslant 2$. Since the dual graph of $(-2)$-curves on $X_{a}$ has no symmetries (see Figure 23), we have $\operatorname{Aut}\left(X_{a}\right)=$ $\operatorname{Aut}_{n t}\left(X_{a}\right)$. Let $p$ be the quasi-elliptic fibration with singular fibers of type (2III* 2 III). Since the Mordell-Weil group of the Jacobian fibration of $p$ is of order 2 (see [Ito94]) and acts effectively on $X_{a}$, we have $\operatorname{Aut}\left(X_{a}\right) \cong \mathbf{Z} / 2 \mathbf{Z}$.

\subsection{The supersingular case of type $\tilde{E}_{7}+{\tilde{A_{1}}}^{(1)}$}

In this subsection, we give a construction of supersingular Enriques surfaces with dual graph of all (-2)-curves given in Figure 20.

Let $\left(X_{0}, X_{1}, X_{2}\right) \in \mathbf{P}^{2}$ and $(S, T) \in \mathbf{P}^{1}$ be homogeneous coordinates. Consider the surface $R$ in $\mathbf{P}^{2} \times \mathbf{P}^{1}$ defined by

$$
S\left(X_{0}^{2}+a^{3} X_{2}^{2}\right)+T\left(X_{1}^{2}+X_{1} X_{2}+a^{2} X_{0} X_{2}\right)=0, \quad a \in k^{*} .
$$




\section{T. Katsura, S. Kondō And G. Martin}

Note that the projection to $\mathbf{P}^{1}$ defines a fiber space $\pi: R \rightarrow \mathbf{P}^{1}$ whose general fiber is a nonsingular conic. The fiber over the point $(S, T)=\left(a^{4}, 1\right)$ is a union of two lines, denoted by $E_{1}$ and $E_{2}$, and the fiber over the point $(S, T)=(1,0)$ is a double line, denoted by $2 E_{3}$. The line defined by $X_{2}=0$ is a 2 -section, denoted by $F_{0}$, of the fiber space.

The surface $R$ has two rational double points $Q_{i}=\left(\left(\alpha, \beta_{i}, 1\right),(1,0)\right)(i=1,2)$, where $\alpha=\sqrt{a^{3}}$ and the $\beta_{i}$ are the roots of the equation $y^{2}+y+a^{3} \sqrt{a}=0$.

Let $\left(x=X_{0} / X_{2}, y=X_{1} / X_{2}, s=S / T\right)$ be affine coordinates. Define

$$
D=\left(s^{2}+a\right) \frac{\partial}{\partial x}+\left(x^{2}+a^{2} s^{2}\right) \frac{\partial}{\partial y} .
$$

Then $D^{2}=0$; that is, $D$ is 2-closed. A direct calculation shows that $D$ has an isolated singularity at the intersection point of the 2 -section $F_{0}$ and the fiber over the point $(S, T)=(1,0)$. As in the case of the previous section, we blow up the two rational double points and the point of isolated singularity of $D$ successively and finally get a vector field without isolated singularities. The configuration of curves is given in Figure 26.

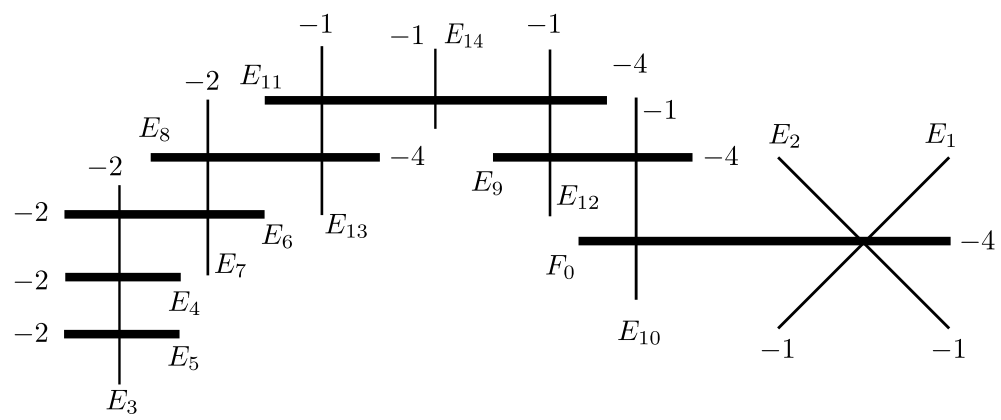

Figure 26

Here, we use the same symbols $F_{0}, E_{1}, E_{2}, E_{3}$ for the curves and their proper transforms, and the suffix $i$ of the other exceptional curve $E_{i}$ corresponds to the order of the successive blow-ups.

We denote by $Y$ the surface obtained by the successive blow-ups. By direct calculations, we have the following lemmas.

Lemma 11.9. (i) The integral curves with respect to $D$ in Figure 26 are all horizontal curves (thick lines).

(ii) $(D)=-\left(F_{0}+4 E_{3}+3 E_{4}+3 E_{5}+4 E_{6}+2 E_{7}+2 E_{8}+2 E_{9}+2 E_{10}+3 E_{11}+4 E_{12}+4 E_{13}+2 E_{14}\right)$.

Lemma 11.10. (i) $(D)^{2}=-12$.

(ii) The canonical divisor $K_{Y}$ of $Y$ is given by

$$
\begin{aligned}
K_{Y}=- & \left(F_{0}+2 E_{3}+E_{4}+E_{5}+2 E_{6}+2 E_{7}+2 E_{8}+2 E_{9}+2 E_{10}\right. \\
& \left.+3 E_{11}+4 E_{12}+4 E_{13}+2 E_{14}\right) .
\end{aligned}
$$

(iii) $K_{Y} \cdot(D)=-4$.

Now take the quotient $Y^{D}$ of $Y$ by $D$. By using the same argument as in the proof of Lemma 7.3, we see that $Y$ is divisorial and hence $Y^{D}$ is non-singular. By Proposition 2.1, we have a configuration of curves as in Figure 27.

Let $X_{a}$ be the surface obtained by contracting the three exceptional curves and the curve meeting the three exceptional curves. The surface $X_{a}$ contains eleven $(-2)$-curves, whose dual 


\section{Classification of EnRiques surfaces}

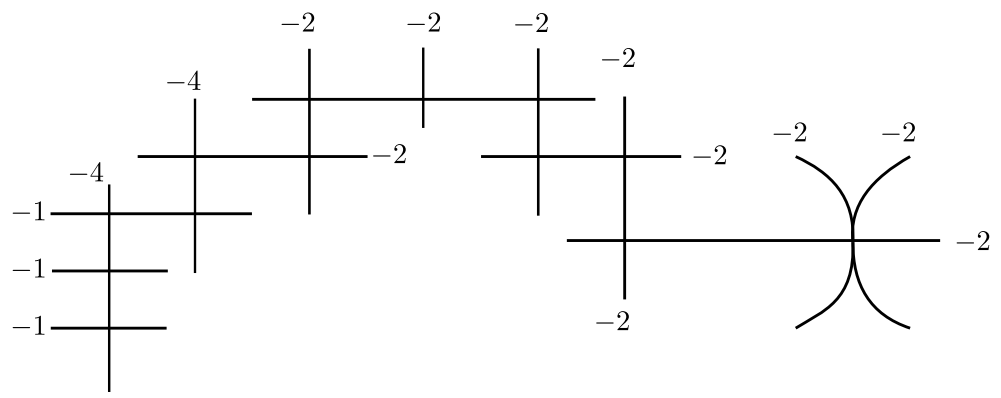

Figure 27

graph is given by Figure 20. Recall that any maximal parabolic subdiagram of this diagram is of type $\tilde{E}_{7} \oplus \tilde{A}_{1}$ or $\tilde{E}_{8}$. By the same argument as in the discussion preceding Theorem 11.4, once we know that $X_{a}$ is an Enriques surface, we can deduce that the surface $X_{a}$ contains a unique quasi-elliptic fibration of type (2III*, III) induced from the fiber space $\pi: R \rightarrow \mathbf{P}^{1}$ and two quasi-elliptic fibrations of type (II*).

TheOREm 11.11. The surfaces $\left\{X_{a}\right\}$ are supersingular Enriques surfaces with dual graph given in Figure 20.

Proof. By using Lemmas 11.9 and 11.10 and the same arguments as in the proofs of Theorems 7.7 and 7.8, we see that $X_{a}$ is an Enriques surface with dual graph given in Figure 20. By construction, the normalization of the canonical cover has a rational double point of type $D_{4}$. It now follows from Lemma 3.7 that $X_{a}$ is supersingular.

In contrast to the previous cases, it is not possible to determine $\operatorname{Aut}\left(X_{a}\right)$ using only the dual graph of (-2)-curves.

THEOREM 11.12. If $a^{7} \neq 1$, then the automorphism group $\operatorname{Aut}\left(X_{a}\right)$ is $\mathbf{Z} / 2 \mathbf{Z}$; it is not numerically trivial. If $a^{7}=1$, then the automorphism group $\operatorname{Aut}\left(X_{a}\right)$ is $\mathbf{Z} / 14 \mathbf{Z}$ and $\operatorname{Aut}_{n t}\left(X_{a}\right)$ is $\mathbf{Z} / 7 \mathbf{Z}$.

Proof. We consider the vector field (11.4), and we set $T=s^{2}, u=x+a s+s^{3}$ and $v=$ $y+s x^{2}+a^{2} s^{3}$. Here, $s=\left(y^{2}+y+a^{2} x\right) /\left(x^{2}+a^{3}\right)$ by (11.3). Then, we have $D(T)=0, D(u)=0$, $D(v)=0$ with the relation

$$
v^{2}+v=T u^{4}+a^{2} u+T^{7},
$$

and the quotient surface $\mathbf{P}^{2}$ by $D$ is birational to the surface defined by the equation (11.5), which is birational to our Enriques surface. For the sake of simplicity, we replace $a^{2}$ with $a$ and consider the change of coordinates with new coordinates $x, y, t$ given by $T=t+a^{4}, v=y+a^{2} x^{2}+a x$, $u=x$. Then, the equation becomes

$$
y^{2}+y=t x^{4}+\left(t+a^{4}\right)^{7} .
$$

This equation gives a non-singular affine chart of a genus 1 fibration $f: X_{a} \rightarrow \mathbf{P}^{1}$ which is a quasi-elliptic fibration (cf. Section 5.2, normal form (2)) with singular fibers of type (2III*, III) by construction. Set

$$
A=k[t, x, y] /\left(y^{2}+y+t x^{4}+\left(t+a^{4}\right)^{7}\right),
$$

and let $\sigma$ be an automorphism of our Enriques surface. The double fiber of $f$ of type III*, denoted by $2 F_{\infty}$, exists over the point defined by $t=\infty$. Since $\sigma$ preserves the diagram of $(-2)$-curves, $\sigma$ preserves $2 F_{\infty}$. Hence $\sigma$ preserves the structure of this quasi-elliptic surface and has the form 


\section{T. Katsura, S. Kondō And G. Martin}

in (6.4) (cf. Remark 6.2). Moreover, this quasi-elliptic surface has a singular fiber over the point defined by $t=0$, and $\sigma$ also preserves the singular fiber. Therefore, we have $\sigma^{*}(t)=c_{1} t$.

Together with the equation (11.6), we have an identity

$$
\begin{aligned}
& e_{1}(t, x)^{2}\left(y+t x^{4}+\left(t+a^{4}\right)^{7}\right)+e_{2}(t, x)^{2}+\left(e_{1}(t, x) y+e_{2}(t, x)\right) \\
& \quad=c_{1} t\left(d_{1}(t) x+d_{2}(t)\right)^{4}+\left(c_{1} t+a^{4}\right)^{7} .
\end{aligned}
$$

The ring $A$ is a free $k[t, x]$-module, and 1 and $y$ are linearly independent over $k[t, x]$. Taking the coefficient of $y$, we have $e_{1}(t, x)^{2}+e_{1}(t, x)=0$. Since $e_{1}(t, x) \neq 0$, we have $e_{1}(t, x)=1$. Hence we have

$$
t x^{4}+\left(t+a^{4}\right)^{7}+e_{2}(t, x)^{2}+e_{2}(t, x)=c_{1} t\left(d_{1}(t) x+d_{2}(t)\right)^{4}+\left(c_{1} t+a^{4}\right)^{7} .
$$

As a polynomial of $x$, if $e_{2}(t, x)$ has a term of degree greater than or equal to 3 , then $e_{2}(t, x)^{2}$ has a term of degree greater than or equal to 6 . We cannot kill this term in the equation. By the equation, we know that $e_{2}(t, x)$ does not have terms in $x$ of odd degree. Therefore, we can put $e_{2}(t, x)=a_{0}(t)+a_{2}(t) x^{2}$ with $a_{0}(t), a_{2}(t) \in k[t]$. We take the coefficients of $x^{4}$ : we have $t+a_{2}(t)^{2}+c_{1} t d_{1}(t)^{4}=0$. Hence we have two equations $1+c_{1} d_{1}(t)^{4}=0$ and $a_{2}(t)^{2}=0$. Thus we have $a_{2}(t)=0$ and $d_{1}(t)=1 / \sqrt[4]{c_{1}}$. The equation becomes

$$
\left(t+a^{4}\right)^{7}+a_{0}(t)^{2}+a_{0}(t)=c_{1} t d_{2}(t)^{4}+\left(c_{1} t+a^{4}\right)^{7} .
$$

Put $\operatorname{deg} d_{2}(t)=\ell$. Suppose $\ell \geqslant 2$. Then, the right-hand side has an odd term whose degree is equal to $4 \ell+1 \geqslant 9$. Therefore, the left-hand side must have an odd term which is of degree $4 \ell+1$. This means $\operatorname{deg} a_{0}(t)=4 \ell+1$. However, in the equation we cannot kill the term of degree $8 \ell+2$ which comes from $a_{0}(t)^{2}$. Therefore, we can put $d_{2}(t)=b_{0}+b_{1} t$ with $b_{0}, b_{1} \in k$. Then, the equation becomes

$$
\left(t+a^{4}\right)^{7}+a_{0}(t)^{2}+a_{0}(t)=c_{1} b_{0}^{4} t+c_{1} b_{1}^{4} t^{5}+\left(c_{1} t+a^{4}\right)^{7} .
$$

If $\operatorname{deg} a_{0}(t) \geqslant 4$, we cannot kill the term of degree greater than or equal to 8 in the equation which comes from $a_{0}(t)^{2}$. Therefore, we can put $a_{0}(t)=\alpha_{0}+\alpha_{1} t+\alpha_{2} t^{2}+\alpha_{3} t^{3}$. Then, we have equations

$$
\begin{gathered}
1=c_{1}^{7}, \quad a^{4}+\alpha_{3}^{2}=c_{1}^{6} a^{4}, \quad a^{8}=c_{1} b_{1}^{4}+c_{1}^{5} a^{8}, \quad a^{12}+\alpha_{2}^{2}=c_{1}^{4} a^{12}, \quad a^{16}+\alpha_{3}=c_{1}^{3} a^{16}, \\
a^{20}+\alpha_{1}^{2}+\alpha_{2}=c_{1}^{2} a^{20}, \quad a^{24}+\alpha_{1}=c_{1} b_{0}^{4}+c_{1} a^{24}, \quad a^{28}+\alpha_{0}^{2}+\alpha_{0}=a^{28} .
\end{gathered}
$$

Assume $a^{7} \neq 1$. Since $\alpha_{3}=\left(c_{1}^{3}+1\right) a^{2}=\left(c_{1}^{3}+1\right) a^{16}$, we have $\left(c_{1}^{3}+1\right) a^{2}\left(a^{7}+1\right)^{2}=0$. By $a^{7} \neq 1$ and $a \neq 0$, we have $c_{1}^{3}=1$. Since $1=c_{1}^{7}$, we have $c_{1}=1$. Therefore, we have $\alpha_{1}=\alpha_{2}=\alpha_{3}=0$, $b_{0}=b_{1}=0$ and $\alpha_{0}=1$ or 0 . Therefore, we see that $\sigma$ is given by either $t \mapsto t, x \mapsto x, y \mapsto y+1$ or the identity. Hence we have $\operatorname{Aut}\left(X_{a}\right) \cong \mathbf{Z} / 2 \mathbf{Z}$ if $a^{7} \neq 1$. Now, assume $a^{7}=1$. From $c_{1}^{7}=1$, we see that $c_{1}$ is a seventh root of unity. We denote by $\zeta$ a primitive seventh root of unity. Then we have a solution

$$
\begin{gathered}
c_{1}=\zeta, \quad \alpha_{1}=0, \quad \alpha_{2}=\left(1+\zeta^{2}\right) a^{6}, \quad \alpha_{3}=\left(1+\zeta^{3}\right) a^{2}, \\
b_{0}=\frac{\left(\zeta^{2}+1\right) a^{6}}{\zeta^{2}}, \quad b_{1}=\frac{\left(\zeta^{3}+1\right) a^{2}}{\zeta^{2}} .
\end{gathered}
$$




\section{Classification of EnRiques surfaces}

We also have $\alpha_{0}=1$ or 0 . Using this data, we have an automorphism $\sigma$ which is defined by

$$
\begin{aligned}
t & \mapsto \zeta t, \\
x & \mapsto \frac{1}{\zeta^{2}} x+\frac{\left(\zeta^{2}+1\right)}{\zeta^{2}} a^{6}+\frac{\left(\zeta^{3}+1\right)}{\zeta^{2}} a^{2} t, \\
y & \mapsto y+1+\left(1+\zeta^{2}\right) a^{6} t^{2}+\left(1+\zeta^{3}\right) a^{2} t^{3} .
\end{aligned}
$$

This $\sigma$ is of order 14, and by our argument, the automorphism group is generated by $\sigma$. This means $\operatorname{Aut}\left(X_{a}\right) \cong \mathbf{Z} / 14 \mathbf{Z}$ if $a^{7}=1$.

Finally, to show $\operatorname{Aut}\left(X_{a}\right) / \operatorname{Aut}_{n t}\left(X_{a}\right) \cong \mathbf{Z} / 2 \mathbf{Z}$, it suffices to show that $X_{a}$ does not admit any numerically trivial involutions, since the symmetry group of the dual graph of $(-2)$-curves is $\mathbf{Z} / 2 \mathbf{Z}$. This is similar to the argument in the proof of Theorem 11.4. By the discussion preceding Theorem 11.11, we know that all genus 1 fibrations on $X_{a}$ are quasi-elliptic. Let $p_{1}$ be the quasielliptic fibration of type (2III*, $\mathrm{III})$, let $p_{2}$ and $p_{3}$ be quasi-elliptic fibrations of type (II*) with cuspidal double fibers $C_{2}$ and $C_{3}$, respectively, and let $g$ be a numerically trivial involution. Note that $C_{2} \cdot C_{3}=1$, as can be read off from the dual graph in Figure 20. Then, $g$ preserves $C_{2}$ and $C_{3}$ and fixes the cusps as well as the intersection $C_{2} \cap C_{3}$ (which is distinct from the cusps since $C_{2} . C_{3}=1$ ). Hence $g$ fixes $C_{2}$ and $C_{3}$ pointwise by Lemma 2.13 (here is where we use that $g$ has order 2). Thus, for a general fiber $F$ of $p_{1}$, the automorphism $g$ fixes the cusp of $F, F \cap C_{2}$ and $F \cap C_{3}$; hence, it fixes $F$ pointwise by Lemma 2.13. This implies that $g$ is trivial, which shows that there is no numerically trivial involution on $X_{a}$.

Remark 11.13. The numerically trivial automorphism $\sigma$ of order 7 found here leads to one of the exceptions in [DM19].

Theorem 11.14. The surfaces $\left\{X_{a}\right\}$ form a 1-dimensional non-isotrivial family of supersingular Enriques surfaces with dual graph given in Figure 20.

Proof. It follows from Theorem 11.12 that not all $\left\{X_{a}\right\}$ are isomorphic. Therefore, the theorem of Matsusaka and Mumford [MM64, Theorem 1] implies that the family $\left\{X_{a}\right\}$ is non-isotrivial.

\section{Enriques surfaces of type $\tilde{D}_{8}$}

In this section, we give a construction of Enriques surfaces with dual graph of all (-2)-curves given in Figure 28.

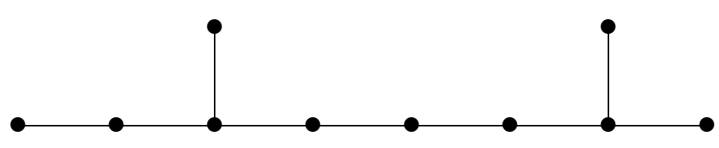

Figure 28

\subsection{Supersingular case}

Let $(x, y)$ be affine coordinates of $\mathbf{A}^{2} \subset \mathbf{P}^{2}$. Consider a rational vector field $D$ defined by

$$
D=D_{a}=\frac{1}{x^{5}}\left(x\left(x^{4}+x^{2}+y^{6}\right) \frac{\partial}{\partial x}+\left(a x^{6}+y\left(x^{4}+x^{2}+y^{6}\right)\right) \frac{\partial}{\partial y}\right),
$$

where $a \in k^{*}$. Then $D^{2}=0$; that is, $D$ is 2-closed. Note that $D$ has poles of order 5 along the line $\ell$ defined by $x=0$, and this line is integral. We see that $D$ has a unique isolated singularity 


\section{T. Katsura, S. Kondō And G. Martin}

$(x, y)=(0,0)$. First, we blow up at the point $(0,0)$. Then we see that the exceptional curve is not integral and the induced vector field has poles of order 2 along the exceptional curve. Moreover, the induced vector field has a unique isolated singularity at the intersection of the proper transform of $\ell$ and the exceptional curve. Continue this process until the induced vector field has no isolated singularities. The final configuration of curves is given in Figure 29. Here, $F_{0}$ is the proper transform of $\ell$, and the suffix $i$ of the exceptional curve $E_{i}$ corresponds to the order of the successive blow-ups.

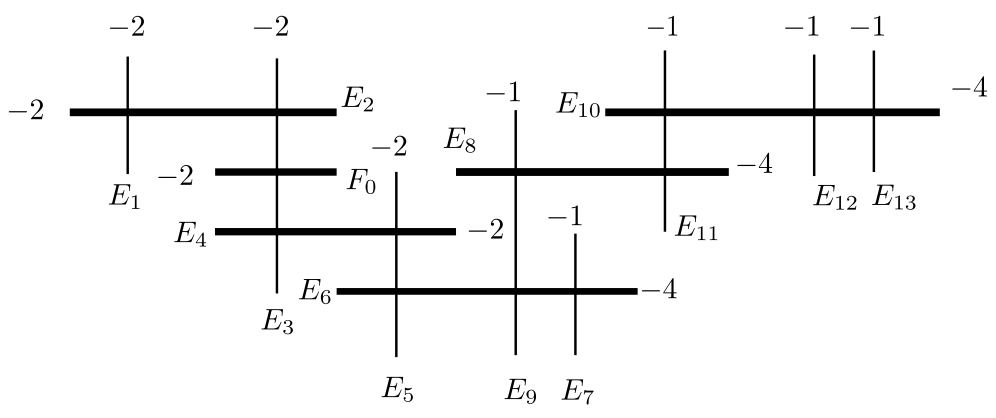

Figure 29

We denote by $Y$ the surface obtained by this process. Also, abusing notation, we denote by $D$ the induced vector field on $Y$. By direct calculations, we have the following lemmas.

Lemma 12.1. (i) The integral curves with respect to $D$ in Figure 29 are all horizontal curves (thick lines).

(ii) $(D)=-\left(5 F_{0}+2 E_{1}+6 E_{2}+8 E_{3}+7 E_{4}+4 E_{5}+3 E_{6}+2 E_{7}+2 E_{8}+4 E_{9}+E_{10}+2 E_{11}\right)$.

LemMa 12.2. (i) $(D)^{2}=-12$.

(ii) The canonical divisor $K_{Y}$ of $Y$ is given by

$$
K_{Y}=-\left(3 F_{0}+2 E_{1}+4 E_{2}+6 E_{3}+5 E_{4}+4 E_{5}+3 E_{6}+2 E_{7}+2 E_{8}+4 E_{9}+E_{10}+2 E_{11}\right) .
$$

(iii) $K_{Y} \cdot(D)=-4$.

Now take the quotient $Y^{D}$ of $Y$ by $D$. By using the same argument as in the proof of Lemma 7.3, we see that $D$ is divisorial and $Y^{D}$ is non-singular. By Proposition 2.1, we have the configuration of curves in Figure 30.

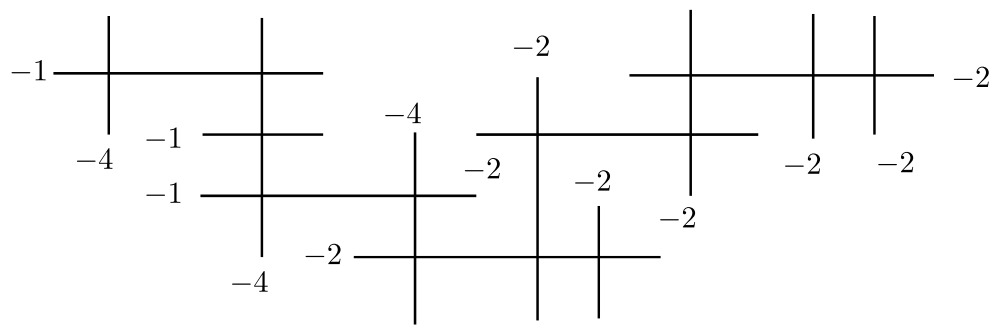

Figure 30

By contracting the three exceptional curves, we get a new exceptional curve which is the image of the $(-4)$-curve meeting three exceptional curves. Let $X_{a}$ be the surface obtained by contracting the new exceptional curve (recall that the vector field (10.1) contains a parameter $a$ ). 


\section{Classification of EnRiques surfaces}

The surface $X_{a}$ contains ten $(-2)$-curves whose dual graph is given by Figure 28. Note that any maximal parabolic subdiagram of this diagram is of type $\tilde{D}_{8}$ or $\tilde{E}_{8}$. Once we know that $X_{a}$ is an Enriques surface, we can use Tables 5 and 6 for one of the genus 1 fibrations with a fiber of type II $^{*}$ to deduce that the leftmost vertex in Figure 28 is part of the conductrix. Hence, by Lemma 3.3 , the genus 1 fibration with a fiber of type $\mathrm{I}_{4}^{*}$ is quasi-elliptic of type $\left(2 \mathrm{I}_{4}^{*}\right)$. This fibration is induced from the pencil of lines in $\mathbf{P}^{2}$ through $(x, y)=(0,0)$.

Theorem 12.3. The surfaces $\left\{X_{a}\right\}$ form a 1-dimensional family of supersingular Enriques surfaces with dual graph given in Figure 28.

Proof. By using Lemmas 12.1 and 12.2 and the same arguments as in the proofs of Theorems 7.7 and 7.8, we see that $X$ is an Enriques surface with dual graph given in Figure 28. By construction, the normalization of the canonical cover has a rational double point of type $D_{4}$. Hence $X_{a}$ is supersingular by Lemma 3.7 .

Remark 12.4. Note that $X_{a}$ contains exactly three genus 1 fibrations. Let $p_{1}$ be the quasi-elliptic fibration with a double singular fiber $2 F_{1}$ of type $\mathrm{I}_{4}^{*}$, and let $p_{i}(i=2,3)$ be two genus 1 fibrations with a singular fiber $F_{i}$ of type II*. Note that, by Table 6 , the conductrix of $X_{a}$ is contained in the singular fiber of type $\mathrm{II}^{*}$ of $p_{2}$ and $p_{3}$. Hence these fibrations are elliptic by Lemma 3.3. Also, note that $F_{1} \cdot F_{2}=F_{1} \cdot F_{3}=F_{2} \cdot F_{3}=2$. If both $F_{2}$ and $F_{3}$ are double fibers, then there are no canonical $U$-pairs on this Enriques surface, which gives a contradiction (Cossec and Dolgachev [CD89, Theorem 3.4.1]). Hence one of them, say $F_{2}$, is double and the other, $F_{3}$, is simple. Since there are no automorphisms which interchange a double fiber and a simple fiber, any automorphism of $X_{a}$ is cohomologically trivial.

ThEOREM 12.5. The automorphism group $\operatorname{Aut}\left(X_{a}\right)$ is the quaternion group $Q_{8}$ of order 8; it is cohomologically trivial.

Proof. We consider the vector field (12.1), and we set $u=x^{2}, v=y^{2}, z=a x^{7}+x^{5} y+x^{3} y+x y^{7}$. Then, we have $D(u)=0, D(v)=0, D(z)=0$ and the equation

$$
z^{2}=a^{2} u^{7}+u^{5} v+u^{3} v+u v^{7} .
$$

Therefore, the quotient surface of $\mathbf{P}^{2}$ by $D$ is birational to the surface defined by the equation (12.2), which is birational to our Enriques surface. To do a change of coordinates, we define new variables $x, y, t$ by $x=1 / u, y=z / u^{4}, t=v / u$, and we replace $a^{2}$ with $a$ for the sake of simplicity. Then, the equation becomes

$$
y^{2}+t x^{4}+t x^{2}+a x+t^{7}=0 .
$$

This equation gives a normal affine chart of a genus 1 fibration $f: X_{a, b} \rightarrow \mathbf{P}^{1}$. Set

$$
A=k[t, x, y] /\left(y^{2}+t x^{4}+t x^{2}+a x+t^{7}\right),
$$

and let $\sigma$ be an automorphism of our Enriques surface. The double fiber of $f$ of type $\mathrm{I}_{4}^{*}$, denoted by $2 F_{\infty}$, exists over the point defined by $t=\infty$. Since $\sigma$ preserves the diagram of $(-2)$-curves, $\sigma$ preserves the curve $C$ of cusps and $2 F_{\infty}$. Thus $\sigma$ has the form in (6.4).

Therefore, together with the equation (12.3), we have an identity

$$
\begin{aligned}
& e_{1}(t, x)^{2}\left(t x^{4}+t x^{2}+a x+t^{7}\right)+e_{2}(t, x)^{2} \\
& =\left(c_{1} t+c_{2}\right)\left(d_{1}(t) x+d_{2}(t)\right)^{4}+\left(c_{1} t+c_{2}\right)\left(d_{1}(t) x+d_{2}(t)\right)^{2}+a\left(d_{1}(t) x+d_{2}(t)\right)+\left(c_{1} t+c_{2}\right)^{7} .
\end{aligned}
$$




\section{T. Katsura, S. Kondō And G. Martin}

Using Lemma 6.3 and taking the coefficients of $x$, we have $a e_{1}(t, x)^{2}+a d_{1}(t)=0$. Therefore, $e_{1}(t, x)$ is a polynomial of $t$; that is, we can put $e_{1}(t, x)=e_{1}(t)$ and $d_{1}(t)=e_{1}(t)^{2}$. Taking the coefficients of $t$, we have

$$
\begin{aligned}
& e_{1}(t)^{2} x^{4}+e_{1}(t)^{2} x^{2}+e_{1}(t)^{2} t^{6}+c_{1}\left(d_{1}(t) x+d_{2}(t)\right)^{4}+c_{1}\left(d_{1}(t) x+d_{2}(t)\right)^{2} \\
& \quad+a d_{2}(t)_{\text {odd }} / t+c_{1}\left(c_{1} t+c_{2}\right)^{6}=0 .
\end{aligned}
$$

Here, $d_{2}(t)_{\text {odd }}$ consists of the odd terms of $d_{2}(t)$. Considering the coefficients of $x^{4}$ of this equation, we have $e_{1}(t)^{2}=c_{1} d_{1}(t)^{4}=c_{1} e_{1}(t)^{8}$. Since $e_{1}(t) \not \equiv 0$, we have $e_{1}(t)^{6}=1 / c_{1}$. Therefore, $e_{1}(t)$ is a constant; we set $e_{1}(t)=e_{1} \in k$. Then, $e_{1}^{6}=1 / c_{1}$. Considering the coefficients of $x^{2}$, we have $e_{1}^{2}=e_{1}(t)^{2}=c_{1} d_{1}(t)^{2}=c_{1} e_{1}^{4}$. Hence $e_{1}^{2}=1 / c_{1}$. Therefore, we have $c_{1}=1$ and so $e_{1}=d_{1}=1$. The equation becomes $t^{6}+d_{2}(t)^{4}+d_{2}(t)^{2}+a d_{2}(t)_{\text {odd }} / t+\left(t+c_{2}\right)^{6}=0$. If the degree of $d_{2}(t)$ is greater than or equal to 2 , then the highest term of $d_{2}(t)^{4}$ cannot be killed in the equation. Therefore, we can put $d_{2}(t)=b_{0}+b_{1} t\left(b_{0}, b_{1} \in k\right)$, and we have an identity

$$
t^{6}+\left(b_{0}+b_{1} t\right)^{4}+\left(b_{0}+b_{1} t\right)^{2}+a b_{1}+\left(t+c_{2}\right)^{6}=0 .
$$

Hence we have $c_{2}=b_{1}^{2}, c_{2}^{2}=b_{1}$ and $b_{0}^{4}+b_{0}^{2}+a b_{1}+c_{2}^{6}=0$. Thus, we have either $c_{2}=0, b_{1}=0$ and $b_{0}=0,1$, or $c_{2}=\omega, b_{1}=\omega^{2}$ and $b_{0}=\alpha$, where $\alpha$ is any root of $z^{2}+z+\omega \sqrt{a}+1=0$. Here, $\omega$ is any cube root of unity. There exist eight solutions. Substituting this into the original equation, we have $e_{2}(t, x)=\sqrt{a}$ or $\omega^{2} x^{2}+\omega^{2} x+\omega^{2} t^{3}+\sqrt{a \alpha}+\sqrt{a}$. Thus, we have

$$
\sigma(t)=t+\omega, \quad \sigma(x)=x+\alpha+\omega^{2} t, \quad \sigma(y)=y+\omega^{2} x^{2}+\omega^{2} x+\omega^{2} t^{3}+\sqrt{a \alpha}+\sqrt{a},
$$

and we conclude $\operatorname{Aut}(X) \cong Q_{8}$. The cohomological triviality follows from Remark 12.4.

Remark 12.6. The group $Q_{8}$, which appears here as the first example of a non-commutative group of cohomologically trivial automorphisms, leads to one of the exceptions in [DM19].

\subsection{Classical case}

Let $Q=\mathbf{P}^{1} \times \mathbf{P}^{1}$ be a non-singular quadric, and let $\left(\left(u_{0}, u_{1}\right),\left(v_{0}, v_{1}\right)\right)$ be homogeneous coordinates of $Q$. Let $x=u_{0} / u_{1}$ and $y=v_{0} / v_{1}$. Consider a rational vector field $D$ defined by

$$
D=\frac{1}{x y^{2}}\left(a x^{2} y^{2} \frac{\partial}{\partial x}+\left(x^{4} y^{4}+b y^{4}+x^{2} y^{2}+x^{2}\right) \frac{\partial}{\partial y}\right)
$$

where $a, b \in k, a, b \neq 0$. Then $D^{2}=a D$; that is, $D$ is 2 -closed. Note that $D$ has a pole of order 1 along the divisor defined by $x=0$, a pole of order 3 along the divisor defined by $x=\infty$ and a pole of order 2 along the divisor defined by $y=0$. Moreover, $D$ has isolated singularities at $(x, y)=(0,0),(\infty, 0)$. As in the case of supersingular Enriques surfaces of type $\tilde{E}_{8}$, we successively blow up the points of isolated singularities of $D$ and finally arrive at a vector field without isolated singularities, which we will denote by $D$ again. The configuration of curves is given in Figure 31 .

Here, $F_{0}, E_{1}$ and $E_{2}$ are the proper transforms of the curves defined by $y=0, x=0$ and $x=\infty$, respectively.

We denote by $Y$ the surface obtained by the successive blow-ups. A direct calculation shows the following two lemmas.

Lemma 12.7. (i) The integral curves with respect to $D$ in Figure 31 are all horizontal curves (thick lines).

(ii) $(D)=-\left(2 F_{0}+E_{1}+3 E_{2}+2 E_{3}+4 E_{5}+4 E_{6}+3 E_{7}+2 E_{8}+2 E_{9}+4 E_{10}+E_{11}+2 E_{12}\right)$.

LEMMA 12.8. (i) $(D)^{2}=-12$. 
Classification of Enriques surfaces

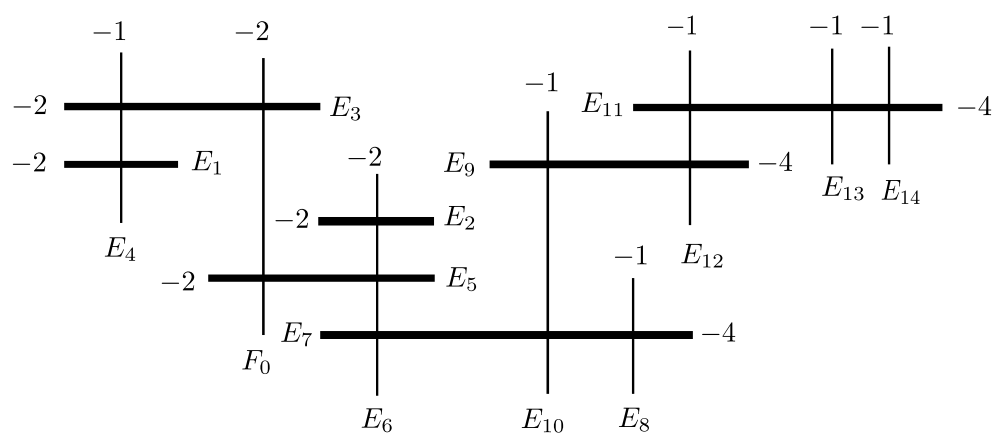

Figure 31

(ii) The canonical divisor $K_{Y}$ of $Y$ is given by

$$
K_{Y}=-\left(2 F_{0}+2 E_{2}+E_{3}+3 E_{5}+4 E_{6}+3 E_{7}+2 E_{8}+2 E_{9}+4 E_{10}+E_{11}+2 E_{12}\right) .
$$

(iii) $K_{Y} \cdot(D)=-4$.

Now take the quotient $Y^{D}$ of $Y$ by $D$. By using the same argument as in the proof of Lemma 7.3, we see that $D$ is divisorial and hence $Y^{D}$ is non-singular. By Proposition 2.1, we have the configuration of curves in Figure 32.

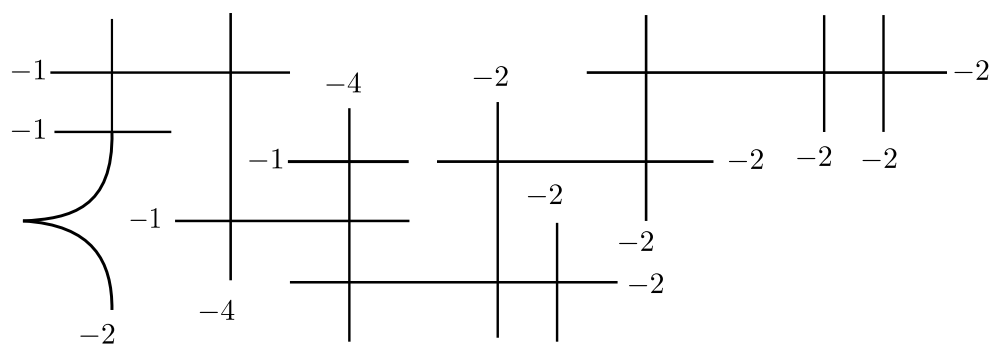

Figure 32

Let $X_{a, b}$ be the surface obtained by contracting the four exceptional curves in Figure 32 (recall that the vector field $D$ contains two parameters $a$ and $b$; see (12.4)). On $X_{a, b}$, there exist ten $(-2)$-curves whose dual graph is given by Figure 28. Recall that any maximal parabolic subdiagram of this diagram is of type $\tilde{D}_{8}$ or $\tilde{E}_{8}$. As in the previous case, once we know that $X_{a, b}$ is an Enriques surface, we can deduce that there exists a quasi-elliptic fibration of type (I*) induced by the first projection from $Q$ to $\mathbf{P}^{1}$.

Theorem 12.9. The surfaces $\left\{X_{a, b}\right\}$ form a 2-dimensional family of classical Enriques surfaces with dual graph given in Figure 28.

Proof. By using Lemmas 12.7 and 12.8 and the same arguments as in the proofs of Theorems 7.7 and 7.8, we see that $X_{a, b}$ is an Enriques surface with dual graph given in Figure 28. Since $X_{a, b}$ has a genus 1 fibration with two double fibers (see Figure 32), $X_{a, b}$ is classical (Proposition 2.5).

Remark 12.10. There are two genus 1 fibrations with a singular fiber of type II*. As we explained in Remark 12.4, one of these fibers is double, the other is simple, and both fibrations are elliptic. If they were of type $\left(\mathrm{II}^{*}\right)$, then their $j$-invariant would be zero (Lang [Lan94]) and hence all non-singular fibers are supersingular elliptic curves by Lemma 2.6. This contradicts the fact that 


\section{T. Katsura, S. Kondō And G. Martin}

a double fiber of a genus 1 fibration on a classical Enriques surface is an ordinary elliptic curve or of additive type (Proposition 2.4). Thus, the two elliptic fibrations are of type (II*, $\mathrm{I}_{1}$ ) by Lang [Lan94].

TheOREM 12.11. The automorphism group $\operatorname{Aut}\left(X_{a, b}\right)$ is $\mathbf{Z} / 2 \mathbf{Z}$; it is numerically trivial.

Proof. It follows from Remark 12.10 that $\operatorname{Aut}\left(X_{a, b}\right)=\operatorname{Aut}_{n t}\left(X_{a, b}\right)$. We consider the vector field (12.4), and we set $u=x^{2}, v=y^{2}, z=x^{5} y^{4}+b x y^{4}+x^{3} y^{2}+x^{3}+a x^{2} y^{3}$. Then, we have $D(u)=0, D(v)=0, D(z)=0$ and the equation

$$
z^{2}=u^{5} v^{4}+b^{2} u v^{4}+u^{3} v^{2}+u^{3}+a^{2} u^{2} v^{3}, \quad a, b \in k^{*} .
$$

Therefore, the quotient surface of $\mathbf{P}^{1} \times \mathbf{P}^{1}$ by $D$ is birational to the surface defined by the equation (12.5), which is birational to our Enriques surface. To do a change of coordinates, we define new variables $x, y, t$ by

$$
x=\sqrt[4]{b} / u v, \quad y=\sqrt[4]{b^{3}} z / u^{4} v^{2}, \quad t=\sqrt{b} / u,
$$

and we replace $1 / \sqrt{b}$ and $a^{2} / \sqrt[4]{b^{5}}$ with $a$ and $b$, respectively, for the sake of simplicity. Then, the equation becomes

$$
y^{2}+t x^{4}+a t^{3} x^{2}+b t^{3} x+t^{3}+t^{7}=0 .
$$

This equation gives a normal affine chart of a genus 1 fibration $f: X_{a, b} \rightarrow \mathbf{P}^{1}$. Set

$$
A=k[t, x, y] /\left(y^{2}+t x^{4}+a t^{3} x^{2}+b t^{3} x+t^{3}+t^{7}\right),
$$

and let $\sigma$ be an automorphism of our Enriques surface. The double fiber of $f$ of type $\mathrm{I}_{4}^{*}$, denoted by $2 F_{\infty}$, exists over the point defined by $t=\infty$. Since $\sigma$ preserves the dual graph of $(-2)$-curves, $\sigma$ preserves the curve $C$ of cusps and $2 F_{\infty}$. Therefore, $\sigma$ has the form in (6.4). Moreover, this quasi-elliptic surface has a singular fiber over the point defined by $t=0$, and the automorphism $\sigma$ also preserves the singular fiber. Therefore, we know $c_{2}=0$, and we have $\sigma^{*}(t)=c_{1} t$.

Therefore, together with the equation (12.6), we have an identity

$$
\begin{aligned}
& e_{1}(t, x)^{2}\left(t x^{4}+a t^{3} x^{2}+b t^{3} x+t^{3}+t^{7}\right)+e_{2}(t, x)^{2} \\
& \quad=c_{1} t\left(d_{1}(t) x+d_{2}(t)\right)^{4}+a\left(c_{1} t\right)^{3}\left(d_{1}(t) x+d_{2}(t)\right)^{2}+b\left(c_{1} t\right)^{3}\left(d_{1}(t) x+d_{2}(t)\right)+\left(c_{1} t\right)^{3}+\left(c_{1} t\right)^{7} .
\end{aligned}
$$

Differentiate both sides with respect to $x$, and we have $b e_{1}(t, x)^{2} t^{3}+b c_{1}^{3} d_{1}(t) t^{3}=0$, that is, $e_{1}(t, x)^{2}=c_{1}^{3} d_{1}(t)$. Therefore, $e_{1}(t, x)$ is a polynomial of $t$; that is, we can put $e_{1}(t, x)=e_{1}(t)$ and $d_{1}(t)=c_{1}^{-3} e_{1}(t)^{2}$. Using Lemma 6.3 and taking the coefficients of $t$, we have

$$
\begin{aligned}
& e_{1}(t, x)^{2}\left(x^{4}+a t^{2} x^{2}+t^{2}+t^{6}\right)+c_{1}\left(c_{1}^{-3} e_{1}(t)^{2} x+d_{2}(t)\right)^{4} \\
& \quad+a c_{1}^{3} t^{2}\left(c_{1}^{-3} e_{1}(t)^{2} x+d_{2}(t)\right)^{2}+b c_{1}^{3} d_{2}(t)_{\text {even }} t^{2}+c_{1}^{3} t^{2}+c_{1}^{7} t^{6}=0 .
\end{aligned}
$$

Here, $d_{2}(t)_{\text {even }}$ consists of the even terms of $d_{2}(t)$. Considering the coefficients of $x^{4}$ of this equation, we have $e_{1}(t)^{2}=c_{1}^{-11} e_{1}(t)^{8}$. Since $e_{1}(t) \not \equiv 0$, we have $e_{1}(t)^{6}=c_{1}^{11}$. Therefore, $e_{1}(t)$ is a constant; we set $e_{1}(t)=e_{1} \in k$. Then, we have $e_{1}^{6}=c_{1}^{11}$. Considering the coefficients of $x^{2}$ of this equation, we have $a e_{1}^{2} t^{2}=a c_{1}^{-3} e_{1}^{4} t^{2}$, that is, $e_{1}^{2}=c_{1}^{3}$. Hence we have $c_{1}^{9}=c_{1}^{11}$. Since $c_{1} \neq 0$, we have $c_{1}=1$. Therefore, we have $e_{1}=1$ and $d_{1}(t)=1$. Then, the equation becomes $d_{2}(t)^{4}+a t^{2} d_{2}(t)^{2}+b d_{2}(t)_{\text {even }} t^{2}=0$. If the degree of $d_{2}(t)$ is greater than or equal to 2 , then the highest term of $d_{2}(t)^{4}$ cannot be killed in the equation. Therefore, we can put $d_{2}(t)=b_{0}+b_{1} t$ $\left(b_{0}, b_{1} \in k\right)$ and we have an identity $\left(b_{0}+b_{1} t\right)^{4}+a\left(b_{0}+b_{1} t\right)^{2} t^{2}+b b_{0} t^{2}=0$. Hence we have $b_{1}^{4}=a b_{1}^{2}$, $a b_{0}^{2}=b b_{0}$ and $b_{0}^{4}=0$. Thus, we have $b_{0}=0$, and $b_{1}=\sqrt{a}$ or 0 . Going to the original equality, we have $e_{2}(t, x)^{2}=b t^{3} \sqrt{a} t$, that is $e_{2}(t, x)=\sqrt[4]{a} \sqrt{b} t^{2}$. Therefore, we conclude that $\sigma$ is given by either $t \mapsto t, x \mapsto x+\sqrt{a} t, y \mapsto y+\sqrt[4]{a} \sqrt{b} t^{2}$ or the identity. Hence we have $\operatorname{Aut}(X) \cong \mathbf{Z} / 2 \mathbf{Z}$. 


\section{Classification of EnRiques surfaces}

\section{Enriques surfaces of type $\tilde{D}_{4}+\tilde{D}_{4}$}

In this section, we give a construction of Enriques surfaces with graph of all (-2)-curves given in Figure 33.

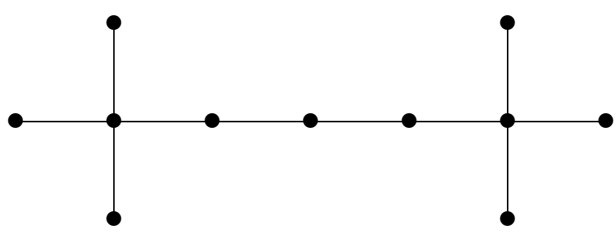

Figure 33

Let $Q=\mathbf{P}^{1} \times \mathbf{P}^{1}$ be a non-singular quadric, and let $\left(\left(u_{0}, u_{1}\right),\left(v_{0}, v_{1}\right)\right)$ be homogeneous coordinates of $Q$. Let $x=u_{0} / u_{1}, x^{\prime}=u_{1} / u_{0}, y=v_{0} / v_{1}, y^{\prime}=v_{1} / v_{0}$. Consider a rational vector field $D$ defined by

$$
D=\frac{1}{x^{2} y^{2}}\left(b x^{3} y^{2} \frac{\partial}{\partial x}+\left(a x^{2} y^{2}+x^{2}+x^{4} y^{4}+y^{4}+b x^{2} y^{3}\right) \frac{\partial}{\partial y}\right),
$$

where $a, b \in k, b \neq 0$. Note that $D^{2}=b D$; that is, $D$ is 2-closed. Denote by $E_{1}, E_{2}$ and $F_{0}$ the curves defined by $x=0, x^{\prime}=0$ and $y=0$, respectively. The vector field $D$ has poles of order 2 along $E_{1}, E_{2}$ and $E_{3}$, and has isolated singularities $(x, y)=(0,0)$ and $\left(x^{\prime}, y\right)=(0,0)$. The curves $E_{1}, E_{2}$ are integral. Now blow up at the two points $(x, y)=(0,0)$ and $\left(x^{\prime}, y\right)=(0,0)$. The two exceptional curves are integral with respect to the induced vector field. The induced vector field has poles of order 3 along two exceptional curves and has isolated singularities at the intersections of the exceptional curves and the proper transforms of $E_{1}$ and $E_{2}$. Then, blow up at the isolated singularities of the induced vector field and continue this process until the induced vector field has no isolated singularities. We denote by $Y$ the surface obtained by this process and use the same symbols $E_{1}, E_{2}, F_{0}$ for the curves and their proper transforms. Also, we denote by the same symbol $D$ the induced vector field on $Y$. The final configuration of curves is given in Figure 34 .

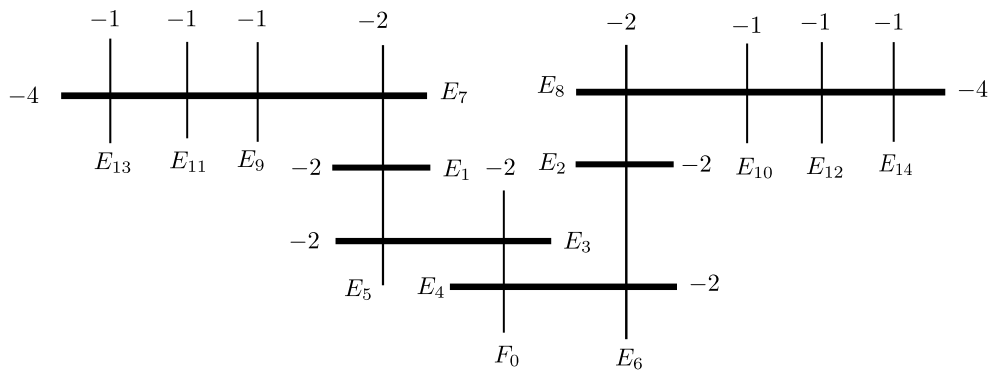

Figure 34

Lemma 13.1. (i) The integral curves with respect to $D$ in Figure 34 are all horizontal curves (thick lines).

(ii) $(D)=-\left(2 F_{0}+2 E_{1}+2 E_{2}+3 E_{3}+3 E_{4}+2 E_{5}+2 E_{6}+E_{7}+E_{8}\right)$.

LemMa 13.2. (i) $(D)^{2}=-12$. 


\section{T. Katsura, S. Kondō And G. Martin}

(ii) The canonical divisor $K_{Y}$ of $Y$ is given by

$$
K_{Y}=-\left(2 F_{0}+E_{1}+E_{2}+2 E_{3}+2 E_{4}+2 E_{5}+2 E_{6}+E_{7}+E_{8}\right) .
$$

(iii) $K_{Y} \cdot(D)=-4$.

Now take the quotient $Y^{D}$ of $Y$ by $D$. By using the same argument as in the proof of Lemma 7.3, we see that $D$ is divisorial and $Y^{D}$ is non-singular. By Proposition 2.1, we have Figure 35 .

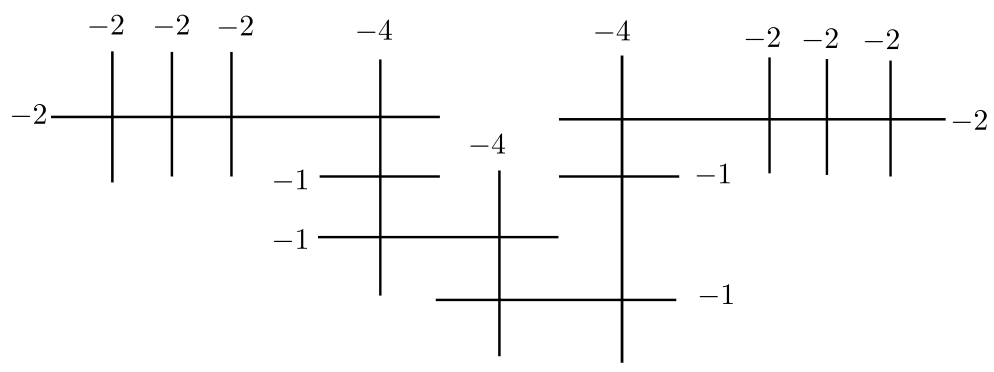

Figure 35

Let $X_{a, b}$ be the surface obtained by contracting the four exceptional curves; it contains eleven $(-2)$-curves, whose dual graph is given by Figure 33. Note that any maximal parabolic subdiagram of this diagram is of type $\tilde{D}_{8}$ or $\tilde{D}_{4} \oplus \tilde{D}_{4}$. The surface $X_{a, b}$ contains a quasi-elliptic fibration $p_{1}$ with singular fibers of type $\left(2 \mathrm{I}_{0}^{*}, 2 \mathrm{I}_{0}^{*}\right)$ induced from the first projection from $Q$ to $\mathbf{P}^{1}$ and nine genus 1 fibrations with a singular fiber of type $\left(\mathrm{I}_{4}^{*}\right)$. These nine genus 1 fibrations are elliptic, as can be seen by comparing to the conductrix given in Ekedahl and Shepherd-Barron [ES04, Theorems 2.2 and 3.1] (see Tables 5 and 6 in the Section 4).

Theorem 13.3. The surfaces $\left\{X_{a, b}\right\}$ form a 2-dimensional family of classical Enriques surfaces with dual graph given in Figure 33. It contains an at least 1-dimensional non-isotrivial family.

Proof. By using Lemmas 13.1 and 13.2 and the same arguments as in the proofs of Theorems 7.7 and 7.8, we see that $X_{a, b}$ is an Enriques surface with dual graph given in Figure 33.

By equation (5.1) in Section 5.3, the surface $X_{a, b}$ is the quasi-elliptic surface given affinely by the equation

$$
u^{2}+S v^{4}+a^{2} S^{3} v^{2}+b^{2} S^{4} v+S^{3}+S^{7}=0 .
$$

By Queen [Que71, Theorem 2], its Jacobian is the quasi-elliptic surface given by

$$
u^{2}+S v^{4}+a^{2} S^{3} v^{2}+b^{2} S^{4} v=0 .
$$

Now we change coordinates

$$
Y=\frac{u}{b S^{2} v^{2}}, \quad X=\frac{1}{v}+\frac{a^{2}}{b^{2} S}, \quad T=\frac{1}{S},
$$

which yields

$$
Y^{2}=X^{3}+\left(a^{4} / b^{4}\right) T^{2} X+\left(1 / b^{2}\right) T^{3} .
$$

Since these Jacobian quasi-elliptic surfaces form a 1-dimensional non-isotrivial family by Ito [Ito94], the family $\left\{X_{a, b}\right\}$ contains an at least 1-dimensional non-isotrivial family.

Theorem 13.4. The automorphism group $\operatorname{Aut}\left(X_{a, b}\right)$ is isomorphic to $(\mathbf{Z} / 2 \mathbf{Z})^{3}$. Moreover, $\operatorname{Aut}_{\text {nt }}\left(X_{a, b}\right) \cong(\mathbf{Z} / 2 \mathbf{Z})^{2}$. 


\section{Classification of Enriques surfaces}

Proof. We use the equation of $X_{a, b}$ given in Theorem 13.3. We set $x=v, y=u, t=S$, and we replace $a^{2}$ and $b^{2}$ with $a$ and $b$, respectively, for the sake of simplicity. Then, the equation becomes

$$
y^{2}+t x^{4}+a t^{3} x^{2}+b t^{4} x+t^{3}+t^{7}=0 .
$$

This equation gives a normal affine surface. Set

$$
A=k[t, x, y] /\left(y^{2}+t x^{4}+a t^{3} x^{2}+b t^{4} x+t^{3}+t^{7}\right) .
$$

Our quasi-elliptic surface $\varphi: X_{a, b} \rightarrow \mathbf{P}^{1}$ has two double fibers of type $\mathrm{I}_{0}^{*}$ over the points defined by $t=0$ and $t=\infty$. First, we consider an automorphism $\tau$ of $X_{a, b}$ defined by

$$
\tau: t \mapsto 1 / t, \quad x \mapsto x / t^{2}, \quad y \mapsto y / t^{5} .
$$

This automorphism is of order 2 and exchanges the two double fibers. Let $\sigma$ be an automorphism of our Enriques surface. Then $\sigma$ either preserves the double fibers or exchanges them. If $\sigma$ exchanges the double fibers, then we consider $\tau \circ \sigma$. This preserves the double fibers. Therefore, we assume that $\sigma$ preserves the double fibers. Since $\sigma$ preserves the diagram of $(-2)$-curves, $\sigma$ preserves the curve $C$ of cusps and the double fiber $2 F_{\infty}$ over $t=\infty$. Therefore, $\sigma$ has the form in (6.4). Moreover, by our assumption, $\sigma$ preserves the double fiber over the point defined by $t=0$. Therefore, we may assume $\sigma^{*}(t)=c_{1} t$. Using these data, we have an identity

$$
\begin{aligned}
e_{1}(t, x)^{2} & \left(t x^{4}+a t^{3} x^{2}+b t^{4} x+t^{3}+t^{7}\right)+e_{2}(t, x)^{2} \\
= & c_{1} t\left(d_{1}(t) x+d_{2}(t)\right)^{4}+a\left(c_{1} t\right)^{3}\left(d_{1}(t) x+d_{2}(t)\right)^{2}+b\left(c_{1} t\right)^{4}\left(d_{1}(t) x\right. \\
& \left.+d_{2}(t)\right)+\left(c_{1} t\right)^{3}+\left(c_{1} t\right)^{7} .
\end{aligned}
$$

Using Lemma 6.3 and taking the coefficients of $x$, we have $b e_{1}(t, x)^{2} t^{4}+b c_{1}^{4} t^{4} d_{1}(t)=0$. Therefore, we have $e_{1}(t, x)^{2}+c_{1}^{4} d_{1}(t)=0$, and $e_{1}(t, x)$ is a polynomial of $t$; that is, we can put $e_{1}(t, x)=e_{1}(t)$ and $d_{1}(t)=e_{1}(t)^{2} / c_{1}^{4}$. Taking the coefficients of $t$, we have

$$
\begin{aligned}
& e_{1}(t)^{2} x^{4}+a e_{1}(t)^{2} t^{2} x^{2}+e_{1}(t)^{2} t^{2}+e_{1}(t)^{2} t^{6}+c_{1}\left(d_{1}(t) x+d_{2}(t)\right)^{4} \\
& \quad+a c_{1}^{3} t^{2}\left(d_{1}(t) x+d_{2}(t)\right)^{2}+b c_{1}^{4} t^{4} d_{2}(t)_{\text {odd }} / t+c_{1}^{3} t^{2}+c_{1}^{7} t^{6}=0 .
\end{aligned}
$$

Here, $d_{2}(t)_{\text {odd }}$ collects the odd terms of $d_{2}(t)$. Considering the coefficients of $x^{4}$ of this equation, we have $e_{1}(t)^{2}=c_{1} d_{1}(t)^{4}=e_{1}(t)^{8} / c_{1}^{15}$. Since $e_{1}(t) \not \equiv 0$, we have $e_{1}(t)^{6}=c_{1}^{15}$. Therefore, $e_{1}(t)$ is a constant; we set $e_{1}(t)=e_{1} \in k$. Then, $e_{1}^{6}=c_{1}^{15}$. Considering the coefficients of $x^{2}$, we have $a t^{2} e_{1}^{2}=a c_{1}^{3} t^{2} d_{1}(t)^{2}=a c_{1}^{3} t^{2}\left(e_{1}^{2} / c_{1}^{4}\right)^{2}=a t^{2} e_{1}^{4} / c_{1}^{5}$. Therefore, $e_{1}^{2}=c_{1}^{5}$ and $d_{1}(t)=c_{1}$. The equation becomes

$$
e_{1}^{2} t^{2}+e_{1}^{2} t^{6}+c_{1} d_{2}(t)^{4}+a c_{1}^{3} t^{2} d_{2}(t)^{2}+b c_{1}^{4} t^{4} d_{2}(t) \text { odd } / t+c_{1}^{3} t^{2}+c_{1}^{7} t^{6}=0 .
$$

If $\operatorname{deg} d_{2}(t) \geqslant 2$, then we cannot kill the highest term of $c_{1} d_{2}(t)^{4}$ in the equation.

Therefore, we can put $d_{2}(t)=b_{0}+b_{1} t$, and we have equations

$$
e_{1}^{2}=c_{1}^{7}, \quad c_{1} b_{1}^{4}+a c_{1}^{3} b_{1}^{2}+b c_{1}^{4} b_{1}=0, \quad e_{1}^{2}+a c_{1}^{3} b_{0}^{2}+c_{1}^{3}=0, \quad c_{1} b_{0}^{4}=0 .
$$

Solving these equations with $e_{1}^{2}=c_{1}^{5}$, we have $b_{0}=0, c_{1}=e_{1}=d_{1}=1$, and $b_{1}$ is either 0 or a root of the equation $z^{3}+a z+b=0$. Putting this data into the original equation, we have $e_{2}(t, x)=0$. Hence we have four automorphisms, which are the identity and

$$
\sigma_{\alpha}(t)=t, \quad \sigma_{\alpha}(x)=x+\alpha t, \quad \sigma_{\alpha}(y)=y,
$$

where $\alpha$ is a root of the equation $z^{3}+a z+b=0$. By a direct calculation, we can prove that the involution $\tau$ and these automorphisms commute with each other. We now conclude $\operatorname{Aut}(X) \cong(\mathbf{Z} / 2 \mathbf{Z})^{3}$. 


\section{T. Katsura, S. Kondō And G. Martin}

Obviously, $\tau$ is not numerically trivial. We show that any involution $\sigma$ preserving each double fiber of type $\mathrm{I}_{0}^{*}$ is numerically trivial. Let $F$ be a double fiber of type $\mathrm{I}_{0}^{*}$, and let $E$ be the component with multiplicity 2 of $F$. Then $\sigma$ preserves $E$ and a simple component $C$ of $F$ meeting with the special 2-section of the fibration, and hence it preserves one more simple component $C^{\prime}$ of $F$. This implies that $\sigma$ fixes two points on $E$ which are intersection points of $E$ with $C$ and $C^{\prime}$. Therefore, $\sigma$ fixes $E$ pointwise, and hence $\sigma$ preserves all components of $F$. Thus $\sigma$ is numerically trivial.

Remark 13.5. The group $\operatorname{Aut}_{\mathrm{nt}}\left(X_{a, b}\right) \cong(\mathbf{Z} / 2 \mathbf{Z})^{2}$ is the first example of a non-cyclic group of numerically trivial automorphisms of a classical Enriques surface.

\section{Appendix}

\section{A.1 Genus 1 fibrations}

We summarize genus 1 fibrations on each of the Enriques surfaces in Theorems 1.3(b) and 1.4(b). The list holds not only for the above examples but also for any Enriques surface with the same dual graph of $(-2)$-curves. We indicate that it is either elliptic or quasi-elliptic after the type of singular fibers.

- Type $\tilde{E}_{8}:\left(2 \mathrm{II}^{*}\right)$ (quasi-elliptic)

- Type $\tilde{E}_{7}+\tilde{A}_{1}^{(1)}$ supersingular: (2III*, $\mathrm{III})$ (quasi-elliptic), (II*) (quasi-elliptic) classical: (2III* $\mathrm{III})$ (quasi-elliptic), (II*) (quasi-elliptic)

- Type $\tilde{E}_{7}+\tilde{A}_{1}^{(2)}:\left(2 \mathrm{III}^{*}, 2 \mathrm{III}\right)$ (quasi-elliptic), (II*) (quasi-elliptic)

- Type $\tilde{E}_{6}+\tilde{A}_{2}$ : supersingular: $\left(2 \mathrm{IV}^{*}, \mathrm{IV}\right)$ (elliptic), (III*, 2III) (quasi-elliptic) classical: $\left(2 \mathrm{IV}^{*}, \mathrm{I}_{3}, \mathrm{I}_{1}\right)$ (elliptic), (III*, 2III) (quasi-elliptic)

- Type $\tilde{D}_{8}$ supersingular: (2I $\left.\mathrm{I}_{4}^{*}\right)$ (quasi-elliptic), (2II*) (elliptic), (II*) (elliptic) classical: $\left(2 \mathrm{I}_{4}^{*}\right)$ (quasi-elliptic), (2II*, $\left.\mathrm{I}_{1}\right)$ (elliptic), (II*, $\left.\mathrm{I}_{1}\right)$ (elliptic)

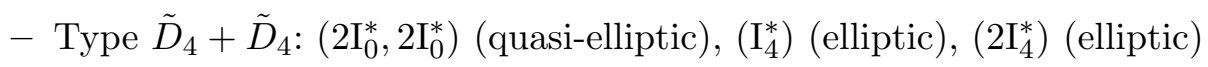

- Type VII: ( $\left.\mathrm{I}_{9}, \mathrm{I}_{1}, \mathrm{I}_{1}, \mathrm{I}_{1}\right)$ (elliptic), ( $\left.\mathrm{I}_{8}, 2 \mathrm{III}\right)$ (elliptic), $\left(\mathrm{I}_{5}, \mathrm{I}_{5}, \mathrm{I}_{1}, \mathrm{I}_{1}\right)$ (elliptic), $\left(\mathrm{I}_{6}, 2 \mathrm{IV}, \mathrm{I}_{2}\right)$ (elliptic)

- Type VIII: $\left(2 \mathrm{I}_{1}^{*}, \mathrm{I}_{4}\right)$ (elliptic), (I $\left.\mathrm{I}_{2}^{*}, 2 \mathrm{III}, 2 \mathrm{III}\right)$ (quasi-elliptic), $\left(\mathrm{IV}^{*}, \mathrm{I}_{3}, \mathrm{I}_{1}\right)$ (elliptic)

\section{A.2 List of examples of equations and automorphisms}

In the following cases, we calculated equations of Enriques surfaces to determine their automorphism groups: type $\tilde{E}_{6}+\tilde{A}_{2}$, supersingular; type $\tilde{E}_{8}$, supersingular and classical; type $\tilde{D}_{8}$, supersingular and classical; type $\tilde{D}_{4}+\tilde{D}_{4}$. In this appendix, we give equations for the remaining examples of Enriques surfaces in Theorems 1.3(b) and 1.4(b).

(1) Enriques surfaces of type $\tilde{E}_{6}+\tilde{A}_{2}$, classical case:

$$
y^{2}+c^{2} t x y+\beta c^{3} t^{2} y=t x^{4}+c^{2} t^{3} x^{2}+\left(c^{3} t^{4}+c^{5} \alpha t^{3}\right) x+t^{7}+t^{3},
$$

where $c=1 /\left(a+\sqrt[4]{a^{3}}\right)$ with $(a \in k \backslash\{0,1\}), \alpha$ is a root of $z^{8}+z^{6}+z^{5}+a^{2} z^{4}+a^{4} z^{3}+$ $a^{8} z^{2}+a^{16}=0$ and $\beta=\left(\alpha^{2}+a^{4}\right) / \alpha$ 


\section{Classification of Enriques surfaces}

(2) Enriques surfaces of type VII:

$$
\begin{aligned}
y^{2}+ & t(t+1)\left(t+a^{2}\right)\left(t+b^{2}\right) x y+\{(a b+1) t+a b\}(t+1)\left(t+a^{2}\right)\left(t+b^{2}\right) y \\
= & t x^{4}+\{(a b+1) t+a b\}(t+1)\left(t+a^{2}\right)\left(t+b^{2}\right) x^{3} \\
& +\left\{t^{2}+(t+1)\left(t+a^{2}\right)\left(t+b^{2}\right)\right\}(t+1)\left(t+a^{2}\right)\left(t+b^{2}\right) x^{2} \\
& +\{(a b+1) t+a b\} t(t+1)\left(t+a^{2}\right)\left(t+b^{2}\right) x+t^{3}+t^{3}(t+1)\left(t+a^{2}\right)\left(t+b^{2}\right) \\
& +t(t+1)^{2}\left(t+a^{2}\right)^{2}\left(t+b^{2}\right)^{2}+t(t+1)^{3}\left(t+a^{2}\right)^{3}\left(t+b^{2}\right)^{3},
\end{aligned}
$$

where $a, b \in k, a+b=a b, a^{3} \neq 1$

(3) Enriques surfaces of type VIII:

$$
y^{2}=t x^{4}+a t^{2} x^{3}+a t^{3}(t+1)^{2} x+t^{7}+t^{3}, \quad a \in k^{*}
$$

(4) Enriques surfaces of type $\tilde{E}_{7}+\tilde{A}_{1}^{(1)}$, classical case:

$$
y^{2}+a t^{2} y=t x^{4}+b t^{3} x+t^{7}+t^{3}, \quad a, b \in k^{*}
$$

(5) Enriques surfaces of type $\tilde{E}_{7}+\tilde{A}_{1}^{(2)}$ :

$$
y^{2}+a t^{2} y=t x^{4}+t^{7}+t^{3}, \quad a \in k^{*}
$$

\section{ACKNOWLEDGEMENTS}

The authors thank I. Dolgachev for stimulating discussions on Enriques surfaces in positive characteristics. The second author thanks N. Shepherd-Barron for explaining the conductrices, and G. van der Geer for discussions and his hospitality when the second author was visiting in Oberwolfach and Amsterdam in 2016. The third author thanks his Ph.D. advisor C. Liedtke for his support and helpful discussions, and the second author for an invitation to Nagoya University in November 2016. The authors thank the referee for the careful reading of the manuscript, for pointing out errors and for many useful suggestions, which greatly improved the readability of this article.

\section{REFERENCES}

BM76 E. Bombieri and D. Mumford, Enriques' classification of surfaces in char. p III, Invent. Math. 35 (1976), 197-232; doi:10.1007/BF01390138.

BP83 W. Barth and C. Peters, Automorphisms of Enriques surfaces, Invent. Math. 73 (1983), no. 3, 383-411; doi:10.1007/BF01388435.

CD89 F. R. Cossec and I.V. Dolgachev, Enriques surfaces. I, Progress in Math., vol. 76 (Birkhäuser Boston, Inc., Boston, MA, 1989); doi:10.1007/978-1-4612-3696-2.

Cos85 F. R. Cossec, On the Picard group of Enriques surfaces, Math. Ann. 271 (1985), no. 4, 577-600; doi:10.1007/BF01456135.

DM19 I. V. Dolgachev and G. Martin, Numerically trivial automorphisms of Enriques surfaces in characteristic 2, J. Math. Soc. Japan 71 (2019), no. 4, 1181-1200; doi:10.2969/jmsj/78867886.

Dol84 I. V. Dolgachev, On automorphisms of Enriques surfaces, Invent. Math. 76 (1984), no. 1, 163-177, doi:10.1007/BF01388499.

Dol13 , Numerical trivial automorphisms of Enriques surfaces in arbitrary characteristic, in Arithmetic and Geometry of K3 Surfaces and Calabi-Yau Threefolds, Fields Inst. Commun., vol. 67 (Springer, New York, 2013), 267-283; doi:10.1007/978-1-4614-6403-7_8. 


\section{T. Katsura, S. Kondō And G. Martin}

ES04 T. Ekedahl and N. I. Shepherd-Barron, On exceptional enriques surfaces, 2004, arXiv:math.AG/ 0405510.

Fan10 G. Fano, Superficie algebriche di genere zero e bigenere uno e loro casi particolari, Rend. Circ. Mat. Palermo 29 (1910), 98-118.

Ill79 L. Illusie, Complexe de de Rham-Witt et cohomologie cristalline, Ann. Sci. École Norm. Sup. 12 (1979), no. 4, 501-661; doi:10.24033/asens.1374.

Ito92 H. Ito, The Mordell-Weil groups of unirational quasi-elliptic surfaces in characteristic 3, Math. Z. 211 (1992), no. 1, 1-39; doi:10.1007/BF02571415.

Ito94_, The Mordell-Weil groups of unirational quasi-elliptic surfaces in characteristic 2 , Tohoku Math. J. 46 (1994), no. 2, 221-251; doi:10.2748/tmj/1178225759.

Ito02 On extremal elliptic surfaces in characteristic 2 and 3, Hiroshima Math. J. 32 (2002), no. 2, 179-188; doi:10.32917/hmj/1151007555.

Kat82 T. Katsura, A note on Enriques' surfaces in characteristic 2, Compos. Math. 47 (1982), no. 2, 207-216; http://www.numdam.org/item?id=CM_1982_-47_2_207_0.

KK15 T. Katsura and S. Kondō, A 1-dimensional family of Enriques surfaces in characteristic 2 covered by the supersingular K3 surface with Artin invariant 1, Pure Appl. Math. Q. 11 (2015), no. 4, 683-709; doi:10.4310/PAMQ.2015.v11.n4.a6.

KK18_, Enriques surfaces in characteristic 2 with a finite group of automorphisms, J. Algebraic Geom. 27 (2018), no. 1, 173-202; doi:10.1090/jag/697.

Kon86 S. Kondō, Enriques surfaces with finite automorphism groups, Japan. J. Math. 12 (1986), no. 2, 191-282; doi:10.4099/math1924.12.191.

KT89 T. Katsura and Y. Takeda, Quotients of abelian and hyperelliptic surfaces by rational vector fields, J. Algebra 124 (1989), no. 2, 472-492, doi:10.1016/0021-8693(89)90144-0.

Lan88 W. E. Lang, On Enriques surfaces in characteristic p. II, Math. Ann. 281 (1988), no. 4, 671-685; doi:10.1007/BF01456844.

Lan91 , Extremal rational elliptic surfaces in characteristic p. I. Beauville surfaces, Math. Z. 207 (1991), no. 3, 429-437; doi:10.1007/BF02571400.

Lan94_, Extremal rational elliptic surfaces in characteristic p. II. Surfaces with three or fewer singular fibres, Ark. Mat. 32 (1994), no. 2, 423-448; doi:10.1007/BF02559579.

Lan00_, Configurations of singular fibres on rational elliptic surfaces in characteristic two, Comm. Algebra 28 (2000), no. 12, 5813-5836; doi:10.1080/00927870008827190.

Lie15 C. Liedtke, Arithmetic moduli and lifting of Enriques surfaces, J. reine angew. Math. 706 (2015), 35-65; doi:10.1515/crelle-2013-0068.

Mar20 G. Martin, Enriques surfaces with finite automorphism group in positive characteristic, Algebraic Geom. 6 (2019), no. 5, 592-649; doi:10.14231/AG-2019-027.

Mat18 Y. Matsumoto, Canonical coverings of Enriques surfaces in characteristic 2, 2018, arXiv:1812. 06914.

MM64 T. Matsusaka and D. Mumford, Two fundamental theorems on deformations of polarized varieties, Amer. J. Math. 86 (1964), 668-684; doi:10.2307/2373030.

MN84 S. Mukai and Y. Namikawa, Automorphisms of Enriques surfaces which act trivially on the cohomology groups, Invent. Math. 77 (1984), no. 3, 383-397; doi:10.1007/BF01388829.

Muk10 S. Mukai, Numerically trivial involutions of Kummer type of an Enriques surface, Kyoto J. Math. 50 (2010), no. 4, 889-902; doi:10.1215/0023608X-2010-017.

Nam85 Y. Namikawa, Periods of Enriques surfaces, Math. Ann. 270 (1985), no. 2, 201-222; doi:10. $1007 / \mathrm{BF} 01456182$.

Nik84 V.V. Nikulin, On a description of the automorphism groups of Enriques surfaces, Soviet Math. Dokl. 30 (1984), 282-285. 


\section{Classification of EnRiques surfaces}

Que71 C.S. Queen, Non-conservative function fields of genus one. I, Arch. Math. (Basel) 22 (1971), 612-623; doi:10.1007/BF01222625.

Que72 , Non-conservative function fields of genus one. II, Arch. Math. (Basel) 23 (1972), 30-37; doi:10.1007/BF01304838.

RS76 A. N. Rudakov and I. R. Shafarevich, Inseparable morphisms of algebraic surfaces, Izv. Akad. Nauk SSSR Ser. Mat. 40 (1976), no. 6, 1269-1307.

Sal03 P. Salomonsson, Equations for some very special enriques surfaces in characteristic two, 2003, arXiv:math.AG/0309210.

Sch17 S. Schröer, Enriques surfaces with normal K3-like coverings, 2017, arXiv:1703.03081.

Vin75 E. B. Vinberg, Some arithmetical discrete groups in Lobačevskiı spaces, Discrete Subgroups of Lie Groups and Applications to Moduli (Internat. Colloq., Bombay, 1973) (Oxford Univ. Press, Bombay, 1975), 323-348.

Toshiyuki Katsura tkatsura@ms.u-tokyo.ac.jp

Graduate School of Mathematical Sciences, The University of Tokyo, Meguro-ku, Tokyo 1538914, Japan

Shigeyuki Kondō kondo@math.nagoya-u.ac.jp

Graduate School of Mathematics, Nagoya University, Nagoya, 464-8602, Japan

Gebhard Martin gmartin@math.uni-bonn.de

Mathematisches Institut, Universität Bonn, Endenicher Allee 60, 53115 Bonn, Germany 